TRANSACTIONS OF THE

AMERICAN MATHEMATICAL SOCIETY

Volume 348, Number 9, September 1996

\title{
KRULL-SCHMIDT THEOREMS IN DIMENSION 1
}

\author{
LAWRENCE S. LEVY AND CHARLES J. ODENTHAL
}

\begin{abstract}
Let $\Lambda$ be a semiprime, module-finite algebra over a commutative noetherian ring $R$ of Krull dimension 1 . We find necessary and sufficient conditions for the Krull-Schmidt theorem to hold for all finitely generated $\Lambda$ modules, and necessary and sufficient conditions for the Krull-Schmidt theorem to hold for all finitely generated torsionfree $\Lambda$-modules (called " $\Lambda$-lattices" in integral representation theory, and "maximal Cohen-Macaulay modules" in the dimension-one situation in commutative algebra).
\end{abstract}

\section{INTRODUCTION}

Throughout this paper $\Lambda$ denotes a semiprime (no nonzero nilpotent ideals) module-finite $R$-algebra, where $R$ is a commutative, noetherian ring of Krull dimension 1 . We say that $\Lambda$ satisfies KSM ("Krull-Schmidt for modules") if, whenever

$$
M_{1} \oplus M_{2} \oplus \ldots \oplus M_{m} \cong N_{1} \oplus N_{2} \oplus \ldots \oplus N_{n}
$$

with each $M_{i}, N_{j}$ an indecomposable, f.g. (finitely generated) left $\Lambda$-module, then we must have $m=n$ and, after a suitable renumbering of the $N_{i}$, every $M_{i} \cong N_{i}$.

We say that $\Lambda$ satisfies TFKS ("torsionfree Krull-Schmidt") if this same conclusion follows whenever each $M_{i}, N_{i}$ in (1.0.1) is torsionfree (see Definitions 1.2), besides being f.g. and indecomposable. The answer to the question of which of our rings $\Lambda$ satisfy KSM or TFKS is, to borrow a well-known phrase, "hardly ever." The precise statements of our main results require some preliminary definitions.

Let $Q=Q(R)$ denote the finite set of minimal prime ideals of $R$. Since $\Lambda$ is semiprime, the localization $\Lambda_{Q}$ obtained by using the denominator set $R-\bigcup Q$ is a semisimple artinian ring. We call $\Lambda$ an $R$-order in the semisimple artinian ring $\Lambda_{Q}$ if the natural map $\Lambda \rightarrow \Lambda_{Q}$ is one-to-one. When this condition holds, we define a normalization $\Gamma$ of $\Lambda$ (in $\Lambda_{Q}$ ) to be a maximal element of the family of subrings of $\Lambda_{Q}$ that contain $\Lambda$ and are integral over $R$. In the classical situation, where $\Lambda$ is contained in a maximal $R$-order in $\Lambda_{Q}$, the normalization $\Gamma$ is easily seen to be such a maximal order. And when $\Lambda$ is commutative, $\Gamma$ becomes a normalization of $\Lambda$ in the sense of commutative ring theory, that is, the integral closure in the total quotient ring. In all cases, $\Gamma$ turns out to be a direct sum of classical maximal orders over Dedekind domains in simple artinian rings, and thus provides a critical link between our theory and classical integral representation theory. See [LO, §4].

Our general $\Lambda$ has a decomposition $\Lambda=\Lambda^{\prime} \oplus A$ where $\Lambda^{\prime}$ is an $R$-order in the semisimple artinian ring $\left(\Lambda^{\prime}\right)_{Q}$ and the $\operatorname{ring} A$ is artinian. [See (2.0.1) below.] Since

Received by the editors April 11, 1994 and, in revised form, September 25, 1995.

1991 Mathematics Subject Classification. Primary 16P40; Secondary $13 E 05$.

Key words and phrases. Krull-Schmidt, unique decomposition.

Levy's research was partially supported by NSF and NSA grants. 
artinian rings always satisfy KSM, the summand $A$ is of no interest in this paper. In particular, if $\Lambda$ is indecomposable (as a ring) and not artinian, it is automatically an $R$-order in $\Lambda_{Q}$.

Recall that the genus of a f.g. $\Lambda$-module $M$ is the collection of all f.g. $\Lambda$-modules $N$ such that $N_{\mathbf{m}} \cong M_{\mathbf{m}}$ for every maximal ideal $\mathbf{m}$ of $R$. This definition is independent of the ring $R$ over which $\Lambda$ is a module-finite algebra [GL '89, 1.3]. In particular, replacing $R$ by the center $Z(\Lambda)$ does not change the definition of the genus of a f.g. $\Lambda$-module. We can now state our first main result, and there is no loss of generality in assuming that $\Lambda$ is an indecomposable ring.

Theorem 1.1 (KSM). Let $\Lambda$ be non-artinian and indecomposable as a ring (and thus an $R$-order in the semisimple artinian ring $\Lambda_{Q}$ ); and let $\Gamma$ be a normalization of $\Lambda$. Then $\Lambda$ satisfies (left) KSM if and only if the following three conditions hold.

(a) Every genus of f.g. (left) $\Lambda$-modules consists of a single isomorphism class.

(b) Either $\Lambda=\Gamma$ or exactly one maximal ideal $\mathbf{n}$ of $Z(\Lambda)$ is singular with respect to $\Lambda$, that is, $\Lambda_{\mathbf{n}} \neq \Gamma_{\mathbf{n}}$ (localizations of $\Lambda, \Gamma$ ).

(c) If this singular $\mathbf{n}$ occurs, then primitive idempotents of $\Gamma_{\mathbf{n}}$ remain primitive modulo $J\left(\Gamma_{\mathbf{n}}\right)$.

Note that $\Gamma_{\mathbf{n}}$ is always a direct sum of full matrix rings over noncommutative principal ideal domains. Part (c) of the (KSM) theorem states that these domains are local, that is, they are division rings modulo their Jacobson radicals.

A matrix-local ring is a ring $B$ such that the ring $B / J(B)[J(B)$ the Jacobson radical] is a simple artinian ring. For the rings that we deal with, this is equivalent to saying that $B$ has a unique maximal (2-sided) ideal [LO, 1.6]. Observe too, that the maximal ideals of $R$ that are singular with respect to $\Lambda$ are independent of the choice of normalization $\Gamma$ [LO, 4.3 and (4.1.7)].

In this terminology, part (c) of the (KSM) theorem states that $\Gamma_{\mathbf{n}}$ is a direct sum of matrix-local rings, and the size of these matrices does not increase when one passes to $\Gamma_{\mathbf{n}} / J\left(\Gamma_{\mathbf{n}}\right)$.

Our original impetus to study the KS problem came from a paper of E. G. Evans [E 73]. He asked which commutative, noetherian local rings satisfy KSM. Our theorem answers his question when the local ring has Krull dimension 1 and no nilpotent elements. In that situation, only condition (c) is nontrivial and, in more conventional commutative terminology, it states that $\Gamma$ is a direct sum of local domains.

Evans showed that a noetherian local ring $S$ is henselian if and only if all of its module-finite algebras satisfy KSM, and he asked whether KSM for $S$ itself is somehow related to the henselian condition (which is too strong a restriction since discrete valuation domains satisfy KSM but need not be henselian). It is interesting, in this regard, that our condition (c) may be viewed as a refinement of the condition that $\Lambda$ be henselian.

If $\Lambda$ is only semilocal (still commutative) it is even easier to construct counterexamples to KSM. For then if $\Lambda$ is an indecomposable ring and has at least two singular maximal ideals, condition (b) fails and so therefore does KSM.

If $R$ is not semilocal, then condition (a) becomes nontrivial. We remark that this condition is not as satisfactory as (b) and (c), because determining that $\Lambda$ satisfies this condition seems to require examining all f.g. $\Lambda$-modules.

Next we discuss TFKS. The theorem is quite similar to the KSM theorem. Condition (a) must be satisfied — but only for torsionfree modules — and condition 
(b) remains unchanged. Condition (c) contains two exceptional classes of rings that satisfy TFKS while violating KSM. But these classes are so small that we can give quite a detailed description of the rings in them. (This description unfortunately makes the statement of the theorem very long.) Perhaps the most unexpected fact is that the exceptional classes (c2) and (c3) can occur only if $\Gamma$ is module-finite over $R$, that is, only if $\Gamma$ is a maximal $R$-order. Before stating our TFKS result, we pause to define "torsionfree" and to introduce terminology needed to describe one of the exceptional classes of rings.

Definitions 1.2. Assume that $\Lambda$ is an $R$-order in a semisimple artinian ring. We say that a $\Lambda$-module $M$ is torsionfree if the natural map $M \rightarrow M_{Q}$ is one-toone. This definition is independent of the particular ring $R$ over which $\Lambda$ is a module-finite algebra because it is equivalent to the assertion that $M$ is a submodule of a free $\Lambda$-module. [Every module over the semisimple artinian ring $\Lambda_{Q}$ is a submodule of a free $\Lambda_{Q}$-module. A denominator-clearing argument then shows that $M$ is a submodule of a free $\Lambda$-module. Conversely, if $M$ is such a submodule, the "order" hypothesis shows that every free $\Lambda$-module is torsionfree, hence so are its $\Lambda$-submodules.]

The equalizer of two ring homomorphisms $f, g: \Gamma \rightarrow \Psi$ is defined to be

$$
\mathrm{eq}(\Gamma, f, g)=\{x \in \Gamma \mid f(x)=g(x)\} .
$$

A "PID" is an integral domain in which every left ideal and every right ideal is principal.

Theorem 1.3 (TFKS). Let $\Lambda$ be non-artinian and indecomposable as a ring (and thus an $R$-order in the semisimple artinian ring $\Lambda_{Q}$ ); and let $\Gamma$ be a normalization of $\Lambda$. Then $\Lambda$ satisfies (left) TFKS if and only if the following conditions are satisfied.

(a) Every genus of f.g. torsionfree $\Lambda$-modules consists of a single isomorphism class.

(b) Either $\Lambda=\Gamma$ or exactly one maximal ideal $\mathbf{n}$ of $Z(\Lambda)$ is singular with respect to $\Lambda$ (i.e. $\Lambda_{\mathbf{n}} \neq \Gamma_{\mathbf{n}}$ ).

(c) If this singular $\mathbf{n}$ occurs, then either condition (c1) holds,

(c1) Primitive idempotents of $\Gamma_{\mathbf{n}}$ remain primitive modulo $J\left(\Gamma_{\mathbf{n}}\right)$, that is, $\Lambda_{\mathbf{n}}$ satisfies KSM,

or else $\Gamma$ is module-finite over $R, \Lambda=M_{m}\left(\Lambda^{\prime}\right)$ and $\Gamma=M_{m}\left(\Gamma^{\prime}\right)$ for some $m$, where $\Lambda^{\prime} \subset \Gamma^{\prime}$ and $\Gamma^{\prime}$ is a PID; and either condition (c2) or (c3) holds.

(c2) $\Gamma_{\mathbf{n}}^{\prime}$ has exactly two maximal ideals $M \neq N$, and $\Gamma_{\mathbf{n}}^{\prime} / M$ and $\Gamma_{\mathbf{n}}^{\prime} / N$ are division rings. Moreover, $\Lambda_{\mathbf{n}}^{\prime}$ is an equalizer $\Lambda_{\mathbf{n}}^{\prime}=\mathrm{eq}\left(\Gamma_{\mathbf{n}}^{\prime}, f, g\right)$ for some surjective $f, g$ such that $\operatorname{ker}(f)=M^{h}$ and $\operatorname{ker}(g)=N^{h}$ for some $h \geq 1$.

(c3) (abstract description) $\Gamma_{\mathbf{n}}^{\prime}$ has exactly one maximal ideal $M$, and $\Gamma_{\mathbf{n}}^{\prime} / M \cong$ $M_{2}(\Delta)$ for some division ring $\Delta$. Moreover $\Gamma_{\mathbf{n}}^{\prime}\left(M \cap \Lambda_{\mathbf{n}}^{\prime}\right) \Gamma_{\mathbf{n}}^{\prime}=M$, and the simple left $\Gamma_{\mathbf{n}}^{\prime}$-module remains simple as a $\Lambda_{\mathbf{n}}^{\prime}$-module.

Remarks 1.4. (i) [on condition (a)]. It is possible for every genus of torsionfree $\Lambda$-modules to consist of a single isomorphism class without the same being true for genera of non-torsionfree modules. An example is given in [KL '90]. In this example TFKS holds and KSM fails; in fact, direct-sum cancellation fails for nontorsionfree modules. This example is noncommutative, and we do not know whether commutative examples exist.

(ii) [on exceptional rings (c2)]. Here $\Lambda^{\prime}$ itself is an equalizer. In more detail, let $f, g$ map $\Gamma_{\mathbf{n}}^{\prime}$ onto $\bar{\Gamma} \cong \Gamma_{\mathbf{n}}^{\prime} / M^{h} \cong \Gamma_{\mathbf{n}}^{\prime} / N^{h}$. Then $f$ and $g$ each map $\Gamma^{\prime}$ itself 
onto $\bar{\Gamma}$ and we have $\Lambda^{\prime}=\mathrm{eq}\left(\Gamma^{\prime}, f, g\right)$. This is easily checked locally, using the following two facts. (1) When $\mathbf{m}$ is a maximal ideal of $Z\left(\Lambda^{\prime}\right)$ and $\mathbf{m} \neq \mathbf{n}$ we have $\Lambda_{\mathbf{m}}^{\prime}=\Gamma_{\mathbf{m}}^{\prime}$. Moreover $\bar{\Gamma}_{\mathbf{m}}=0$ because the maximal ideals of $\Gamma$ and $Z(\Gamma)$ are in one-to-one correspondence via contraction [LO, (4.1.6)], and the integral extension $Z\left(\Lambda^{\prime}\right) \subset Z(\Gamma)$ satisfies lying over. (2) Formation of equalizers commutes with localization.

Since $\Lambda=M_{m}\left(\Lambda^{\prime}\right)$ it follows that $\Lambda$ is an equalizer, too. The reason for not displaying $\Lambda$ itself as an equalizer in the statement of (c2) is that in the rest of this paper it will be important to focus on the fact that (c2) involves only locally determined information, that is, properties of $\Lambda_{\mathbf{n}}^{\prime}$.

There is a converse of the preceeding construction: Let $\Lambda^{\prime}$ be an equalizer $\Lambda^{\prime}=$ eq $\left(\Gamma^{\prime}, f, g\right)$ where $\Gamma^{\prime}$ is a principal ideal domain that is a module-finite $R$-algebra and $\operatorname{ker}(f)=M^{h}, \operatorname{ker}(g)=N^{h}$ for distinct maximal ideals $M, N$ of $\Gamma^{\prime}$ and some $h \geq 1$. Then $Z\left(\Lambda^{\prime}\right)$ has exactly one singular maximal ideal, namely $\mathbf{n}=M \cap Z\left(\Lambda^{\prime}\right)=$ $N \cap Z\left(\Lambda^{\prime}\right)$. Moreover, if condition (a) holds, (e.g. if $R$ is semilocal) then $\Lambda^{\prime}$ is an exceptional ring satisfying TFKS. This follows easily by applying Theorem 1.3.

(iii) [on exceptional rings (c3)]. It is not easy to visualize or construct examples of these rings from the abstract description given in Theorem 1.3. Therefore we give a second, explicit description to supplement the foregoing abstract one. In brief: $\Gamma_{\mathbf{n}}^{\prime} / M \cong M_{2}(\Delta)$ for some division ring $\Delta, \Lambda_{\mathbf{n}}^{\prime} /\left(M \cap \Lambda_{\mathbf{n}}^{\prime}\right)$ is again a division ring and has dimension 2 over $\Delta$, and $M \cap \Lambda_{\mathbf{n}}^{\prime}$ contains elements of $M-M^{2}$. See $\S 11$ for full details.

Finally, we note that the maximal conductor ideal for $\Lambda_{\mathbf{n}}^{\prime}$ and $\Gamma_{\mathbf{n}}^{\prime}$ is some power $M^{n}$. In Section 11 we show that all positive integers $n$ can occur in this way.

Our second impetus to study the KS problem came from a research announcement of H. Jacobinski [J '74]. He was interested in TFKS over the orders $\Lambda$ that occur in integral representation theory. In particular, his $\Lambda$ is contained in a maximal $R$-order $\Gamma$ in $\Lambda_{Q}$. He states necessary and sufficient conditions, together with a sketch of a proof of the necessity of these conditions. Unfortunately, his conditions are not quite correct because his exceptional class (c3) is too small. [In the notation of our remarks on (c3), he thought that $n$ had to equal 1. See our $\S 11$ for examples.] Moreover, his announcement contains no hint of the complicated matrix arguments that we had to use to prove that the exceptional classes (c2) and (c3) actually satisfy TFKS. Our TFKS theorem completes Jacobinski's project, gives a more detailed description of exceptional classes (c2) and (c3), and removes his hypothesis that $\Lambda$ be contained in a maximal order in $\Lambda_{Q}$.

Our work makes extensive use of his ideas, often modified so much that it is not practical to explicitly acknowledge them. For example, we learned the importance of condition (a) from Jacobinski's paper. But in the generality of the present paper, a suitable form of Serre's direct-summand theorem is what we actually use to prove the necessity of condition (a). We also learned the necessity of condition (b) from Jacobinski's paper.

Our third impetus to study the KS problem came from a simple but interesting observation of Reiner [Re '61, Cor. 3]. Assume that $R$ is a discrete valuation ring and $\Lambda$ is a classical order (i.e. contained in a maximal order). Reiner's observation states that a sufficient condition for $\Lambda$ to satisfy KSM (resp. TFKS) is that every f.g. module (resp. torsionfree module) over the $J(R)$-adic completion $\hat{\Lambda}$ of $\Lambda$ be the 
completion of some $\Lambda$-module. This is a main ingredient in the proof of Heller's wellknown sufficient condition for $\Lambda$ to satisfy TFKS: The $J(R)$-induced completion $\hat{V}$ of every simple $\Lambda_{Q}$-module $V$ is a simple $\hat{\Lambda}_{Q}$-module. In fact Heller's original proof obtained some additional information. Implicit in his proof of [H ' $61,2.5]$ is a proof that his sufficient condition implies KSM for $\Lambda$ (not just TFKS), provided that the discrete valuation ring $R$ has characteristic 0 .

Since these criteria are so useful, it is natural to ask whether they are also necessary conditions. But maximal orders offer blatant counterexamples, and therefore not much attention seems to have been given to this type of question. However, one should really ignore maximal orders since they always satisfy KSM when $R$ is semilocal.

In Section 2 we refine these criteria to necessary and sufficient conditions for $\mathrm{KSM}$, we remove the restriction that $\Lambda$ be contained in a maximal order, and we remove the characteristic 0 restriction in Heller's theorem. See Corollary 2.5 and the results that follow it.

The situation for TFKS is different. Neither Reiner's nor (our refinement of) Heller's condition is necessary for TFKS, even for classical orders, because the exceptional rings (c2) and (c3) misbehave. See Remarks 2.12.

Let $R$ be semilocal. As applications of our main results, we show that $\Lambda$ satisfies KSM for left modules if and only it satisfies KSM for right modules. Moreover, if $\Lambda$ satisfies KSM, then so does every $R$-order between $\Lambda$ and $\Gamma$. The same results hold for TFKS. We do not know whether the semilocal hypothesis can be deleted (except that it is trivial that TFKS moves up to larger $R$-orders in $\Lambda_{Q}$ ). The difficulty is that of applying condition (a) of the KSM and TFKS theorems.

One question often asked is whether the KSM property causes endomorphism rings of indecomposable modules to be local (only one maximal left ideal). Of course $R$ must be semilocal in order to make the question natural. A correct, but overly flippant answer is "no, because maximal orders always satisfy KSM when $R$ is semilocal". For a trivial example, one can take the 6 -localization $\Gamma=\mathbb{Z}_{6}$. This is indecomposable as a $\Gamma$-module, but is not a local ring. Even worse, one can easily find a noncommutative maximal order $\Gamma$ that is an integral domain (hence indecomposable), and such that $\Gamma$ maps onto a full matrix ring of size at least $2 \times 2$; and therefore $\Gamma$ is not local.

As an application of our results, we show that maximal orders are responsible for all of the trouble. But to get rid of the trouble one must avoid maximal orders both globally and locally: Assume that $\Lambda$ is not a maximal order, $\Lambda$ satisfies KSM, and $R$ is semilocal. Let $\mathbf{n}$ be the unique maximal ideal of $Z(\Lambda)$ that is singular with respect to $\Lambda$. Then, for every indecomposable f.g. $\Lambda$-module $M$, the endomorphism ring of $M_{\mathbf{n}}$ is a local ring (Corollary 2.11).

The analogous result for TFKS fails because the exceptional rings (c2) and (c3) misbehave. See Remarks 2.12.

Package Deal Game. Let $R$ be semilocal. The method of constructing counterexamples to KS, when it fails, was the same in the papers of Evans and Jacobinski, as well as in papers of Reiner [Re '62, §4] and Roggenkamp [Ro '69] on the subject of $\mathrm{KS}$, and deserves to be formalized and better known. Let $\mathcal{K}$ be a class of (f.g. left) $\Lambda$-modules that is closed under (finite) direct sums and direct summands; and let $\mathcal{M}$ be a class of modules over some other ring, with $\mathcal{M}$ closed under direct sums and direct summands. Let $\Phi: \mathcal{K} \rightarrow \mathcal{M}$ be a function that is additive and faithful in 
the sense that

$$
\Phi(M \oplus N) \cong \Phi(M) \oplus \Phi(N) \text { and } \quad M \cong N \Longleftrightarrow \Phi(M) \cong \Phi(N) .
$$

Suppose also that KS holds in $\mathcal{M}$. Let $M, N$ be indecomposable $\Lambda$-modules in $\mathcal{K}$ whose images under $\Phi$ have decompositions $\Phi(M) \cong U \oplus V$ and $\Phi(N) \cong X \oplus Y$ with $U, V, X, Y$ indecomposable and pairwise nonisomorphic. We call the direct sums $U \oplus V$ and $X \oplus Y$ packages to indicate that they are elements of the image of $\Phi$. We have

$$
(U \oplus V) \oplus(X \oplus Y) \cong(U \oplus X) \oplus(V \oplus Y) .
$$

It might happen that $U \oplus X$ and $V \oplus Y$ are also packages, say $\Phi(A) \cong U \oplus X$ and $\Phi(B) \cong V \oplus Y$. Since $\Phi$ is additive and faithful, we have

$$
M \oplus N \cong A \oplus B .
$$

Since no two of $U, V, X, Y$ are isomorphic to each other, and $\mathrm{KS}$ holds in $\mathcal{M}$, we conclude that KS fails for $\Lambda$-modules in $\mathcal{K}$. We call this procedure the "package deal game". The terminology is chosen to emphasize that, since KS holds in $\mathcal{M}$, all packages $\Phi(M)$ determine their "ingredients" — that is, their uniquely determined indecomposable direct summands (counting multiplicity). It is easy to see that when such a function $\Phi$ exists, all counterexamples to KS in $\mathcal{K}$ arise in this way by reassembling the ingredients of packages as in (1.4.2) - though not necessarily with the number of ingredients used above.

In the papers of Evans, Jacobinski, Reiner, and Roggenkamp mentioned above, $R$ is a local ring and $\Phi(M)$ is the $J(R)$-adic completion of $M$ ( $J=$ Jacobson radical). The same procedure is clearly applicable if $R$ is any semilocal ring. Alternatively, $\Phi$ can be chosen to be other functions. For example, suitable localizations, or modules over the "artinian pair" of rings arising from the bottom line of a conductor square for $\Lambda$ and a maximal order containing it. We make use of all of these.

Normalizations. As mentioned before, we assume in this paper that $\Lambda$ has a semisimple artinian quotient ring $\Lambda_{Q}$. But we do not assume the conventional hypothesis that $\Lambda$ is contained in a maximal $R$-order in $\Lambda_{Q}$, that is, we do not assume that $\Gamma$ is module-finite over $R$. The price of this is that we have to rebuild some of the basic tools of integral representation theory.

For example, when $R$ is semilocal and $\Gamma$ is not module-finite over $R$, the $J(R)$ adic completion $\hat{\Lambda}$ contains nilpotent ideals, so the associated quotient ring $\hat{\Lambda}_{Q}$ is artinian but not semisimple, hence does not have the trivial module theory possessed by semisimple artinian rings. However, it turns out that the only $\hat{\Lambda}_{Q^{-} \text {-modules }}$ that we have to look at are those that are completions of $\Lambda_{Q}$-modules, hence are projective (since $\Lambda_{Q}$ is semisimple). So we are helped by the fact that projective modules have tractable structure over all artinian rings.

One way in which maximal orders $\Gamma$ are used in integral representation theory is that all f.g. torsionfree $\Gamma$-modules are projective; and when $R$ is semilocal the structure of projective $\Gamma$-modules is no more complicated than the structure of modules over the semisimple artinian ring $\Gamma_{Q}$. As a result, many module-theoretic questions are easily answered for $\Gamma$-modules; and one then uses a "conductor square" to descend from $\Gamma$ to $\Lambda$.

When the normalization $\Gamma$ of $\Lambda$ is not module-finite over $R$, conductor ideals for $\Gamma$ and $\Lambda$ do not exist. It turns out that we only need conductor arguments when $R$ is semilocal. Moreover, in studying KS, one never has to look at more than 
a finite number of modules at once (namely, those that occur in some particular pair of decompositions of a given module). When $R$ is semilocal we can find what we call a splitting order $\Omega$ that is module-finite over $R$ and salvages the needed properties of $\Gamma$. KS holds for projective $\Omega$-modules $P$ because the isomorphism and decomposition properties of $P$ are the same as those of the projective module $P_{Q}$ over the artinian ring $\Lambda_{Q}=\Gamma_{Q}$. Moreover, conductor arguments can be used for the pair of rings $\Lambda \subset \Omega$ because $\Omega$ is module-finite over $R$. Finally, given any finite set of torsionfree $\Lambda$-modules $L_{1}, \ldots, L_{n}$, we can find a splitting order $\Omega$ that is large enough so that all of the $\Omega$-modules $\Omega L_{i}$ are projective.

Lack of a maximal order containing $\Lambda$ poses another difficulty. In the rings that occur in integral representation theory, there are only finitely many maximal ideals $\mathbf{m}$ of $R$ such that $\Lambda_{\mathbf{m}}$ is not a maximal $R_{\mathbf{m}}$-order, and this often results in a reduction of module-theoretic problems to the case that $R$ is semilocal. In our more general situation, $\Lambda_{\mathbf{m}}$ might be a nonmaximal order for all $\mathbf{m}$, as shown by a commutative example of Hochster [Ho '73]. However, as in [GL '89] we are able to show that many critical properties are semilocal in nature, anyway. For example, indecomposability is a semilocal property, in the following sense: For any indecomposable f.g. $\Lambda$-module $M$ there is a finite set $\mathcal{M}$ of maximal ideals of $R$ such that the localization at the complement of their union $M_{\mathcal{M}}$ is an indecomposable $\Lambda_{\mathcal{M}}$-module, and remains indecomposable when $\mathcal{M}$ is replaced by any larger finite set of maximal ideals [LO, 2.20]. But, unlike the situations in algebraic geometry and integral representation theory, the set $\mathcal{M}$ varies from module to module.

Those properties (package deal theorems, completions, splitting orders) that do not deal directly with the KS problem are developed in the companion paper [LO], so that the present paper can give a clearer exposition of our solution of the KS problem.

Readers interested only in the classical situation can ignore normalizations by always interpreting $\Gamma$ to be a maximal order. And they can ignore splitting orders by setting $\Omega=\Gamma$ whenever a splitting order $\Omega$ occurs.

The present paper is organized as follows.

Section 2, Main Results. This section begins with the notation that will remain fixed throughout the paper, and then proceeds to the statements and proofs of the main consequences of our KSM and TFKS theorems. The rest of the paper is then devoted to the proofs of these two theorems, and examples.

Section 3, Semilocal Reduction, Semilocal Notation. This section reduces the proofs of the two main theorems to the situation that $R$ is semilocal, and $R$ then remains semilocal for the rest of the paper. At the end of the section we collect the notation for the semilocal situation that remains fixed throughout the rest of the paper.

Section 4, Package Deal Game: Combinatorics. We develop the combinatorial machinery needed to play the package deal game with torsionfree $\Lambda$-modules. The additive faithful function used here sends a torsionfree $\Lambda$-module to a module over the artinian pair $(\bar{\Lambda}, \bar{\Omega})$ of rings that form the bottom line of a conductor square for $\Lambda$ and a splitting order $\Omega$. The spirit is this. Every $(\bar{\Lambda}, \bar{\Omega})$-module $[X \subseteq Y]$ has an "array of ranks" associated with it, one rank for every maximal ideal of the normalization $\Gamma$ of $\Lambda$. This array of ranks determines whether $[X \subseteq Y]$ is a package, that is, arises from a torsionfree $\Lambda$-module. This extends a commutative result of $\mathrm{R}$. Wiegand [W' $89,1.6$ ], and the details are given in [LO, 6.21]. 
Let $\mathcal{S}$ be a class of torsionfree $\Lambda$-modules that is closed under direct sums and direct summands, and such that $\Omega L$ is a projective $\Omega$-module for every $L$ in the class $\mathcal{S}$. (This last condition is automatically satisfied if $\Omega$ is a maximal order.) The main result of the section is a fundamental combinatorial lemma that gives (in terms of the above-mentioned ranks) a necessary and sufficient condition for KS to hold in $\mathcal{S}$.

Section 5, Only One Singular Maximal Ideal. This reduces both KS problems (KSM and TFKS) to the case that the center $Z(\Lambda)$ is a local ring. This occurs in two steps. First we show that if $\Lambda$ is an indecomposable ring and TFKS holds, then there can be at most one maximal ideal $\mathbf{n}$ of $Z(\Lambda)$ such that $\Lambda_{\mathbf{n}} \neq \Gamma_{\mathbf{n}}$. Our main contribution here is to remove Jacobinski's hypothesis that $\Lambda$ is contained in a maximal order. Second, we show that $\Lambda$ satisfies KSM or TFKS if $\Lambda_{\mathbf{n}}$ does. Both steps are accomplished by playing appropriate package deal games.

Section 6, Local KSM, completes the proof of our KSM theorem by proving it when $Z(\Lambda)$ is local. The appropriate package deal game here involves $J(R)$-adic completions of $\Lambda$-modules.

Section 7, Linked and Inertial Maximal Ideals. We develop the noncommutative generalizations of "lying-over" and "inertial" that arise when we compare the maximal ideals of $\Gamma$ with those of $\Lambda$. This makes use of a refined notion of lying over that is more complicated than ordinary intersection, and has played a recent role elsewhere in noncommutative ring theory (see [MR '87, Chap. 10]). We refer to two maximal ideals of $\Gamma$ as " $\Lambda$-linked" if they lie over a common maximal ideal of $\Lambda$, in this new way.

Section 8 , Three indecomposable $(\bar{\Lambda}, \bar{\Omega})$-modules. We construct the specific modules over the artinian pair $(\bar{\Lambda}, \bar{\Omega})$ that we need in order to play the package deal game for torsionfree modules in Section 9.

Section 9, Necessary Conditions for Local TFKS. The TFKS theorem was reduced, in earlier sections, to the situation that $Z(\Lambda)$ is a local ring. This section proves that the conditions given in the theorem are indeed necessary when $Z(\Lambda)$ is local.

Section 10, Sufficient Conditions for Local TFKS. A complicated matrix argument is given here, to prove the sufficiency part of our TFKS theorem when $Z(\Lambda)$ is a local ring.

Section 11, Local TFKS: Explicit Version. We give our "explicit" description of the exceptional rings of type (c3) in the TFKS theorem and use it to construct examples of these rings. In particular we construct an example - a noncommutative integral domain - of the exceptional class of rings that is missing from Jacobinski's classification.

\section{MAin Results}

This section begins with a discussion of notation that will remain fixed throughout the paper. Then we state and prove the main applications of our KSM and TFKS theorems. One can show [GL '89, (1.4.3)]:

(2.0.1) Every semiprime module-finite $R$-algebra $\Lambda^{\prime}$, with $R$ noetherian of dimension 1 , is a direct sum of two $R$-algebras $\Lambda^{\prime}=\Lambda \oplus A$, where the summand $\Lambda$ is an $R$-order in a semisimple artinian ring, and the summand $A$ is a semisimple artinian ring. Moreover, $\Lambda$ has no nonzero $R$-submodules of finite length, and no nonzero $\Lambda$-submodules of finite length. 
The artinian summand $A$ is of no interest in this paper, because it is well-known to satisfy KSM. Therefore the definitions and fixed notation below are chosen to fix our attention on the "order" summand $\Lambda$, for the rest of this paper.

Notation 2.1 (Fixed). We assume, from now on, that our noetherian ring $R$ of dimension 1 has no nilpotent elements $\neq 0$. This is no loss of generality, because the $R$-algebra $\Lambda$ that we are studying is semiprime (has no nilpotent ideals $\neq 0$ ), and hence the subring $R \cdot 1_{\Lambda}$ of $\Lambda$ has no nilpotent elements $\neq 0$. (If $r \in R$ is nilpotent, then $\Lambda r$ is a nilpotent ideal of $\Lambda$.)

$Q=Q(R)$ denotes the finite set of minimal prime ideals of $R$, and $R_{Q}$ denotes the localization obtained by inverting the elements of $R-\cup Q$. Since $R$ has no nilpotent elements, $R-\cup Q$ is the set of regular elements (non-zero-divisors) of $R$, and therefore $R_{Q}$ is the total quotient ring of $R$.

$\Lambda$ always denotes an $R$-order in the semisimple artinian ring $\Lambda_{Q}$, by which we mean that $\Lambda$ is a module-finite $R$-algebra, the localization $\Lambda_{Q}$ is a semisimple artinian ring, and the natural map $\Lambda \rightarrow \Lambda_{Q}$ is one-to-one. This definition is independent of the particular ring $R$ over which $\Lambda$ is a module-finite algebra, because $\Lambda_{Q}$ is the Goldie quotient ring of $\Lambda$. (See also [LO, 1.5].) Whenever convenient, we make the nontriviality assumption:

(2.1.1) The center $Z(\Lambda)$ - equivalently, $\Lambda$ itself — has no artinian ring-direct summands.

This causes no loss of generality, in view of (2.0.1). We do not always assume that $R \subseteq \Lambda$, although in the statement of our main theorems there is no loss of generality in assuming that $R=Z(\Lambda)$.

The term module always means "finitely generated left module" unless the contrary is stated. We denote the direct sum of $n$ copies of a module $U$ by $U^{n}$. We also denote the $n^{\text {th }}$ power of an ideal $M$ by $M^{n}$. The context should make it plain which meaning is intended. For example $\Lambda / M^{n}$ can only denote $\Lambda$ modulo the $n^{\text {th }}$ power of $M$.

Caution. Some of the notational conventions in the companion paper [LO] are more general than the ones stated above. For example, $\Lambda$ and $R$ can sometimes have nilpotent ideals in [LO], and modules need not be f.g. there.

The notation $\Gamma$ always denotes a normalization of $\Lambda$. Recall that this means a maximal element of the family of subrings of $\Lambda_{Q}$ that contain $\Lambda$ and are integral over $R$. The basic properties of normalizations, developed in [GL '89], are summarized in [LO, Section 4] in the form that we shall need them. If $\Lambda$ is contained in a maximal $R$-order in $\Lambda_{Q}$, then any $\Gamma$ becomes a maximal $R$-order in $\Lambda_{Q}$. (See [LO, (4.1.7)].) As in the case of maximal orders, $\Gamma$ obviously has a ring decomposition

$$
\Gamma=\bigoplus_{k} \Gamma_{k}
$$

where each (of the finitely many) $\Gamma_{k}$ is a ring such that $\left(\Gamma_{k}\right)_{Q}$ is a simple ring-direct summand of $\Lambda_{Q}$. Therefore $\Gamma_{k}$ is a prime ring $(A B=0 \Rightarrow A$ or $B=0$ for ideals $A, B)$.

Remark 2.2. If $R$ [hence $Z(\Lambda)$ ] is semilocal, then condition (a) of our KSM and TFKS theorems can be omitted. This holds because, when $R$ is semilocal, "genus" is always the same as "isomorphism class". For one proof of this well-known result, note that the $\operatorname{ring} R^{\prime}=\bigoplus\left\{R_{\mathbf{m}} \mid \mathbf{m} \in \operatorname{maxspec} R\right\}$ is a faithfully flat extension of the semilocal noetherian ring $R$. Therefore Grothendieck's theorem on isomorphism 
by faithfully flat descent [Gr ' $65, \mathrm{IV},(2.5 .8)]$ shows that, for f.g. $\Lambda$-modules $M, N$, the relation $R^{\prime} \otimes_{R} M \cong R^{\prime} \otimes_{R} N$ as $R^{\prime} \otimes_{R} \Lambda$-modules implies $M \cong N$ as $\Lambda$-modules.

The precise significance of condition (c) of our KS theorems is as follows:

Proposition 2.3. Suppose that $Z(\Lambda)$ has exactly one singular maximal ideal $\mathbf{n}$ with respect to $\Lambda$, and $\Lambda$ satisfies condition (a) of the KSM theorem (resp. the TFKS theorem). Then $\Lambda$ satisfies KSM (resp. TFKS) if and only if $\Lambda_{\mathbf{n}}$ does.

Proof. We may assume that $\Lambda$ is an indecomposable ring [since any decomposition of $\Lambda$ also decomposes $Z(\Lambda)$ ] and is not artinian. We claim that $\Lambda_{\mathbf{n}}$ is an indecomposable non-artinian ring.

To see this, note that $Z\left(\Lambda_{\mathbf{n}}\right)=Z(\Lambda)_{\mathbf{n}}$, a local hence indecomposable ring. But indecomposability of the center of any ring implies indecomposability of the ring itself. If $\Lambda_{\mathbf{n}}$ were an artininan ring we would have $\Lambda_{\mathbf{n}}=\left(\Lambda_{\mathbf{n}}\right)_{Q}$. Since $\Lambda \subseteq \Gamma \subseteq \Lambda_{Q}$, this implies that $\Lambda_{\mathbf{n}}=\Gamma_{\mathbf{n}}$ and therefore contradicts singularity of $\mathbf{n}$, completing the proof of the claim.

We are given that $\Lambda$ satisfies conditions (a) and (b) of the KSM or TFKS theorem, and therefore $\Lambda$ satisfies KSM or TFKS if and only if condition (c) holds. In view of the claim above, we can apply the KSM or TFKS theorem a second time, but to $\Lambda_{\mathbf{n}}$ in place of $\Lambda$, to complete the proof of the theorem. This is easily done since condition (a) becomes trivial locally [Remark 2.2], condition (c) is a local condition, and normalizations localize [LO, 4.3].

Applications. We now proceed to the first of the main consequences of our KSM and TFKS theorems.

Corollary 2.4. Suppose that $R$ is a semilocal ring. Then:

(a) KSM holds for right $\Lambda$-modules if and only if it holds for left $\Lambda$-modules.

(b) If KSM holds for $\Lambda$-modules, then it holds for $\Lambda^{\prime}$-modules for every $R$-order $\Lambda^{\prime}$ such that $\Lambda \subseteq \Lambda^{\prime} \subseteq \Lambda_{Q}$.

The analogs of statements (a) and (b) for TFKS also hold.

Proof. In proving this theorem we can ignore condition (a) of our KSM Theorem 1.1, as explained in Remark 2.2.

Statement (a). Since conditions (b) and (c) of the KSM Theorem are obviously left-right symmetric, left KSM holds if and only if right KSM does. The same proof works for TFKS, but uses our TFKS Theorem.

Next we prove statement (b) for the case of KSM. Let $\Lambda \subseteq \Lambda^{\prime} \subseteq \Lambda_{Q}$ where $\Lambda$ is indecomposable and non-artinian, $\mathrm{KSM}$ holds for $\Lambda$, and $\Lambda^{\prime}$ is module-finite over $R$. Let $\Gamma$ be a normalization of $\Lambda^{\prime}$, hence also a normalization of $\Lambda$. Then $\Lambda \subseteq \Lambda^{\prime} \subseteq \Gamma$. Moreover, we can take $R=Z(\Lambda)$. We have $R \subseteq R^{\prime} \subseteq \tilde{R}$ where $R^{\prime}=Z\left(\Lambda^{\prime}\right)$ and $\tilde{R}=Z(\Gamma)$, because $\Lambda_{Q}=\Lambda_{Q}^{\prime}=\Gamma_{Q}$. It is shown in [LO, (4.1.2)] that $\tilde{R}$ is the integral closure of $R$ in $Z\left(\Gamma_{Q}\right)$. We can ignore the trivial case that $\Lambda=\Gamma$. So suppose that the singular $\mathbf{n}$ mentioned in the KSM theorem exists.

Write $\Lambda^{\prime}=\bigoplus_{i} \Lambda_{i}^{\prime}$ with each ring $\Lambda_{i}^{\prime}$ indecomposable. We have to check two properties of each $\Lambda_{i}^{\prime}$, namely the properties in statements (b) and (c) of Theorem 1.1; and we can localize $\Lambda$ at $\mathbf{n}$ before checking them. So, after a change of notation we have that $R=Z(\Lambda)$ is a local ring. (This reduction uses the facts that centers localize (an easy exercise), and normalizations localize [LO, Lemma 4.3].) Each $Z\left(\Lambda_{i}^{\prime}\right)$ is a semilocal ring. We want to check that it has at most one singular 
maximal ideal, as described in condition (b) of Theorem 1.1, and that condition (c) holds if the singular maximal ideal occurs. We dispose of condition (b) by proving:

$$
\text { Each } R_{i}^{\prime}=Z\left(\Lambda_{i}^{\prime}\right) \text { is a local ring. }
$$

First we claim that $\tilde{R}$ is a direct sum of local domains.

We have $\tilde{R}=Z(\Gamma)=\bigoplus_{k} S_{k}$ where $S_{k}=Z\left(\Gamma_{k}\right)$. Each $S_{k}$ is an integral domain, since $\Gamma_{k}$ is a prime ring. Moreover, $\Gamma_{k}$ is matrix-local since $K S M$ holds for $\Lambda$. Since the maximal ideals of $\Gamma_{k}$ are in one-to-one correspondence with those of its center via contraction $[\mathrm{LO},(4.1 .6)]$, we see that $S_{k}$ is a local domain as claimed.

Next we claim that each minimal prime of $R^{\prime}=Z\left(\Lambda^{\prime}\right)$ is contained in a unique maximal ideal of $R^{\prime}$. Let $\mathbf{p}^{\prime}$ be any minimal prime of $R^{\prime}$, and let $\mathbf{m}^{\prime}$ and $\mathbf{n}^{\prime}$ be maximal ideals of $R^{\prime}$ containing $\mathbf{p}^{\prime}$. We may assume that $\mathbf{p}^{\prime}$ is not a maximal ideal (otherwise there is nothing to prove). By the lying-over theorem some prime $\mathbf{p}^{\prime \prime}$ of the integral extension $\tilde{R}$ of $R^{\prime}$ contracts to $\mathbf{p}^{\prime}$. Since $\tilde{R}$ is integral over $R^{\prime}$, the going-up theorem [K '70, Theorem 44] yields prime ideals $\mathbf{m}^{\prime \prime}$ and $\mathbf{n}^{\prime \prime}$ of $\tilde{R}$ that contract to $\mathbf{m}^{\prime}$ and $\mathbf{n}^{\prime}$ respectively, and contain $\mathbf{p}^{\prime \prime}$. Since our rings have dimension 1 we see that $\mathbf{p}^{\prime \prime}$ is a minimal prime of $\tilde{R}$ and $\mathbf{m}^{\prime \prime}, \mathbf{n}^{\prime \prime}$ are maximal ideals. But since $\tilde{R}$ is a direct sum of local domains, each minimal prime of $\tilde{R}$ is contained in a unique maximal ideal. Hence $\mathbf{m}^{\prime \prime}=\mathbf{n}^{\prime \prime}$ and therefore $\mathbf{m}^{\prime}=\mathbf{n}^{\prime}$ as claimed.

Now we prove (2.4.1), focusing on $R_{1}^{\prime}$ for simplicity of notation. We have $R^{\prime}=$ $\bigoplus_{i} R_{i}^{\prime}$; and each $R_{i}^{\prime}=Z\left(\Lambda_{i}^{\prime}\right)$ is an indecomposable ring since any decomposition of it would furnish orthogonal central idempotents that decompose $\Lambda_{i}^{\prime}$. The property that each minimal prime of $R^{\prime}$ is contained in a unique maximal ideal is inherited by $R_{1}^{\prime}$. Let $\mathbf{m}^{\prime}$ be any maximal ideal of $R_{1}^{\prime}$. Let $\mathbf{q}$ be the intersection of all minimal primes of $R_{1}^{\prime}$ contained in $\mathbf{m}^{\prime}$ and $\mathbf{q}^{\prime}$ the intersection of all other minimal primes of $R_{1}^{\prime}$. Since every minimal prime is contained in a unique maximal ideal, no maximal ideal contains both $\mathbf{q}$ and $\mathbf{q}^{\prime}$. Therefore $\mathbf{q}+\mathbf{q}^{\prime}=R_{1}^{\prime}$. Since $R_{1}^{\prime}$ has no nilpotent elements, we have $\mathbf{q} \cap \mathbf{q}^{\prime}=0$, and therefore $R_{1}^{\prime}=\mathbf{q} \oplus \mathbf{q}^{\prime}$. Since $\mathbf{q} \neq R_{1}^{\prime}$, indecomposability of $R_{1}^{\prime}$ shows that $\mathbf{q}=0$, so $\mathbf{q}$ is the intersection of all minimal primes of $R_{1}^{\prime}$. This shows that every maximal ideal of $R_{1}^{\prime}$ contains all minimal primes. If $R_{1}^{\prime}$ had two distinct maximal ideals, this would contradict the fact that every minimal prime is contained in a unique maximal ideal. Thus (2.4.1) is proved.

It remains to verify condition (c) of the KSM theorem for each $\Lambda_{i}^{\prime}$. Again we focus on $i=1$. Since $\Lambda$ satisfies KSM and $Z(\Lambda)$ is local, we can rephrase condition (c) of the KSM theorem in the form: primitive idempotents of each $\Gamma_{k}$ remain primitive modulo $J\left(\Gamma_{k}\right)$. On the other hand, the direct sum of some subset of the $\Gamma_{k}$ is a normalization $\Gamma^{\prime}$ of $\Lambda_{1}^{\prime}$. It follows that primitive idempotents of $\Gamma^{\prime}$ remain primitive modulo $J\left(\Gamma^{\prime}\right)$. In view of (2.4.1) this verifies condition (c) of the KSM theorem for $\Lambda_{1}^{\prime}$, and therefore completes the proof of statement (b) of the present corollary for the case of KSM.

Finally, consider statement (b) for the case of TFKS. This is much simpler than the case of KSM, and is well-known. We include a brief proof for completeness. Merely note two facts. (i) if $M$ and $N$ are torsionfree $\Lambda^{\prime}$-modules that are $\Lambda$-isomorphic, then they are $\Lambda^{\prime}$-isomorphic because any $\Lambda$-isomorphism $f$ can be extended to the $\Lambda_{Q}$-isomorphism $f_{Q}: M_{Q} \cong N_{Q}$. (ii) If $M$ is an indecomposable torsionfree $\Lambda^{\prime}$-module, then it remains indecomposable as a $\Lambda$-module (because any $\Lambda$-projection map can be extended to a $\Lambda_{Q}$-projection map, and then restricted to a $\Lambda^{\prime}$-projection map). 
We do not know whether the corollary holds when $R$ is not semilocal.

On completions. Let $R$ be semilocal. If, for every indecomposable $\Lambda$-module $M$, its $J(R)$-adic completion $\hat{M}$ is an indecomposable $\hat{\Lambda}$-module, then KSM obviously passes down from $\hat{\Lambda}$ to $\Lambda$. We wish to refine this sufficient condition for KSM to a necessary and sufficient condition. We must ignore maximal orders, since they always satisfy KSM $[\mathrm{LO},(4.1 .8)]$. The key case is that in which $Z(\Lambda)$ is a local ring; and we therefore deal with this first. Recall that "splitting order" is defined in [LO, §5], and that for classical orders we may take the splitting order $\Omega=\Gamma$. See 3.5 below for properties of splitting orders.

Corollary 2.5. Suppose that $Z(\Lambda)$ is a local ring and $\Lambda \neq \Gamma$; and let $\Omega$ be a splitting order $(\Lambda \subseteq \Omega \subseteq \Gamma)$. Then the following statements are equivalent.

(i) $\Lambda$ satisfies KSM.

(ii) The $J(R)$-adic completion $\hat{M}$ of every indecomposable $\Lambda$-module $M$ is an indecomposable $\hat{\Lambda}$-module.

(iii) The $J(R)$-adic completion of every indecomposable projective $\Omega$-module is an indecomposable $\hat{\Omega}$-module.

(iv) The $J(R)$-induced completion $\hat{R} \otimes_{R} V$ of every simple $\Lambda_{Q}$-module $V$ is an indecomposable $\hat{\Lambda}_{Q}$-module.

(v) Every $\hat{\Lambda}$-module $X$ such that $X_{Q}$ is $\hat{\Lambda}_{Q}$-projective is the $J(R)$-adic completion of some $\Lambda$-module.

Remarks 2.6. In accordance with our standard notation, the subscript $Q$ in conditions (iv) and (v) denotes the $Q=Q(R)$-localization, that is, the denominator set it uses is $R-\bigcup Q(R)$. The ring $\hat{\Lambda}_{Q}$ [the $Q(R)$-localization of $\left.\hat{\Lambda}\right]$ is always artinian [LO, (3.1.2)]. But when $\Gamma$ is not module-finite over $R$, it has nilpotent ideals. One can also regard $\hat{\Lambda}_{Q}$ as the $J(R)$-induced completion of $\Lambda_{Q}$ since $\hat{\Lambda}_{Q} \cong \hat{R} \otimes_{R} \Lambda_{Q}$. See $[\mathrm{LO}, \S 3$, especially 3.6 , and $\S 6]$ for more about $J(R)$-induced completions of $\Lambda_{Q}$ and its modules, the natural generalization to our context of the completion of a field with respect to a discrete valuation.

When $\Gamma$ is module-finite over $R$ the phrase "such that $X_{Q}$ is $\hat{\Lambda}_{Q}$-projective" can be deleted from condition (v) because, in this classical situation, the artinian ring $\hat{\Lambda}_{Q}$ is semisimple and hence all of its modules are projective.

Proof of Corollary 2.5. We may assume that $R=Z(\Lambda)$, since the $J(R)$-adic completion of every $\Lambda$-module equals its $J(Z(\Lambda))$-adic completion [LO, 6.2]. Next we comment on notation. Let $M_{k i}^{\prime}$ denote a maximal ideal of $\Gamma_{k}$. In [LO, (6.1.2)] we prove that all primitive idempotents of $\Gamma_{k}$ split into the sum of the same number $s_{k i}$ of orthogonal idempotents of $\Gamma_{k} / M_{k i}^{\prime}$. This number $s_{k i}$ is called the "splitting number" of $M_{k i}^{\prime}$ [see also (3.5.2) in the present paper]. In particular the statement that $s_{k i}=1$ means that every primitive idempotent of $\Gamma_{k}$ remains primitive modulo $M_{k i}^{\prime}$.

(i) $\Rightarrow(v)$. Since $\Lambda \neq \Gamma$, the unique maximal ideal of the local $\operatorname{ring} Z(\Lambda)$ is singular with respect to $\Lambda$. Therefore, by our KSM theorem, primitive idempotents of $\Gamma$ itself remain primitive modulo $J(\Gamma)$ and $\Gamma$ is a direct sum of matrix-local rings. Therefore primitive idempotents of each indecomposable ring direct summand $\Gamma_{k}$ of $\Gamma$ remain primitive modulo $J\left(\Gamma_{k}\right)$ and $\Gamma_{k}$ is matrix-local. In particular, for every $k$ there is only one splitting number $s_{k i}$, and this $s_{k 1}=1$. Condition (v) is now part of the conclusion of the package deal theorem for completions of orders [LO, $6.15]$. 
$(\mathrm{v}) \Rightarrow(\mathrm{i})$ is $[\mathrm{LO}, 6.16$, (ii) $\Rightarrow$ (iii)].

(v) $\Rightarrow$ (ii). This is immediate, as soon as one knows that, for every $\Lambda$-module $M, \hat{M}_{Q}$ is a projective $\hat{\Lambda}_{Q}$-module. This latter fact is an easy consequence of the hypothesis that the ring $\Lambda_{Q}$ is semisimple artinian. See the package deal theorem for completions of orders [LO, 6.15].

(ii) $\Rightarrow$ (iii) holds since, for every indecomposable projective $\Omega$-module $P, P_{Q}$ is an indecomposable $\Lambda_{Q}$-module $[\mathrm{LO}, 5.5]$, and hence $P$ is indecomposable as a $\Lambda$-module.

(iii) $\Rightarrow$ (iv). Every indecomposable projective $\Lambda_{Q}$-module has the form $P_{Q}$, where $P$ is an indecomposable projective module over the splitting order $\Omega$ [LO, 5.5]. By (iii), $\hat{P}$ is an indecomposable $\hat{\Omega}$-module. Therefore, by [LO, 6.9 or 6.11 ], $\hat{P}_{Q}$ is an indecomposable $\hat{\Omega}_{Q \text {-module. }}$

(iv) $\Rightarrow(\mathrm{v})$. We claim that every projective $\hat{\Omega}_{Q}$-module $U$ is the $J(R)$-induced completion of some projective $\Omega_{Q}$-module. We may assume that $U$ is indecomposable. We have a decomposition $\Omega_{Q}=\bigoplus_{i} P_{i}$, where each $P_{i}$ is an indecomposable projective $\Omega_{Q}$-module. Tensoring with $\hat{R}$ yields $\hat{\Omega}_{Q}=\bigoplus_{i} \hat{P}_{i}$, where each summand $\left(\hat{P}_{i}\right)_{Q}$ is an indecomposable $\hat{\Omega}_{Q}$-module, by (iv). Since KSM holds for the artinian ring $\hat{\Omega}_{Q}$, every isomorphism class of indecomposable projective $\hat{\Omega}_{Q}$-module occurs in this decomposition, so the claim is proved.

Now let $X$ be a $\hat{\Lambda}$-module such that $X_{Q}$ is $\hat{\Lambda}_{Q}$-projective. We want to prove that $X$ is the completion of some $\Lambda$-module. It suffices, by the package deal theorem for completions [LO, 3.4], to prove that $X_{Q}$ is the $J(R)$-induced completion of some $\Lambda_{Q}$-module. Since $\Lambda_{Q}=\Omega_{Q}$ and both are f.g. $R$-modules, a second application of the package deal theorem for completions shows that it suffices to prove that $X_{Q}$ is the $J(R)$-induced completion of some $\Omega_{Q}$-module; and this was done in the preceding paragraph. [Note how the need to work with $\Omega$ arose when we changed rings. We have $\Lambda_{Q}=\Gamma_{Q}$. But if $\Gamma$ is not module-finite over $R$, we cannot make the second application of the package deal theorem for completions.]

We now state the general form of our necessary and sufficient condition.

Corollary 2.7. Suppose that $R$ is semilocal, $\Lambda$ is an indecomposable ring, and $\Lambda \neq \Gamma$. Then $\Lambda$ satisfies KSM if and only if

(i) $Z(\Lambda)$ has exactly one singular maximal ideal $\mathbf{n}$ with respect to $\Lambda$; and

(ii) The $J\left(R_{\mathbf{n}}\right)$-adic completion of every indecomposable $\Lambda_{\mathbf{n}}$-module is an indecomposable $\hat{\Lambda}_{\mathbf{n}}$-module.

Proof. As in the proof of Corollary 2.5 we may suppose that $R=Z(\Lambda)$. Moreover, $\Lambda$ is not an artinian ring since $\Lambda \neq \Gamma$. By Proposition 2.3, $\Lambda$ satisfies KSM if and only if $\Lambda_{\mathbf{n}}$ does. Since $Z\left(\Lambda_{\mathbf{n}}\right)$ is the local ring $Z(\Lambda)_{\mathbf{n}}$, Corollary 2.5 now completes the proof.

On Heller's theorem. A well-known theorem of A. Heller (implicit in the proof of [H '61, 2.5]) states that if $R$ is a discrete valuation ring of characteristic zero and $\Lambda$ is contained in a maximal order, then condition (i) of the following corollary implies that $\Lambda$ satisfies KSM. The characteristic 0 hypothesis was removed in [CR ' 81 , (30.18)], but only for torsionfree $\Lambda$-modules. The following corollary removes these special hypotheses, and condition (ii) provides a simple explanation of condition (i) in terms of the structure of the ring $\Gamma$. 
Corollary 2.8. The following statements are equivalent, if $R$ is semilocal.

(i) The $J(R)$-induced completion $\hat{R} \otimes_{R} V$ of every simple $\Lambda_{Q}$-module $V$ is an indecomposable $\hat{\Lambda}_{Q}$-module.

(ii) Primitive idempotents of $\Gamma$ remain primitive modulo $J(\Gamma)$.

When the conditions hold, $\Lambda$ satisfies KSM and $Z(\Lambda)$ is a direct sum of local rings.

Proof. We claim that we may suppose that $R=Z(\Lambda)$. For the $J(R)$-adic completion of any $\Lambda$-module equals its $J(Z(\Lambda)$ )-adic completion [LO, 6.2]. Moreover, any isomorphism $\hat{R} \otimes_{R} M \cong Z(\Lambda)^{\wedge} \otimes_{Z(\Lambda)} M$ of completions of a (f.g.) $\Lambda$-module $M$ can be $Q$-localized to obtain an isomorphism of the $J(R)$ - and $J(Z(\Lambda))$-induced completions of $M_{Q}$.

Recall the decomposition (2.1.2). Statement (ii) is equivalent to the assertion that every primitive idempotent of every $\Gamma_{k}$ remains primitive modulo $J\left(\Gamma_{k}\right)$. This, in turn, is equivalent to saying (in our standard notation for maximal ideals of $\Gamma_{k}$ ) that $\Gamma_{k}$ has only one maximal ideal $M_{k 1}^{\prime}$ and primitive idempotents of $\Gamma_{k}$ remain primitive modulo $M_{k 1}^{\prime}=J\left(\Gamma_{k}\right)$. In terms of the splitting numbers $s_{k i}$ defined in the first paragraph of the proof of Corollary 2.5 this can be further restated:

(2.8.1) For each $k$ there is only one splitting number, and $s_{k 1}=1$.

Regardless of whether conditions (i) or (ii) hold, each $\Omega_{k}$ has a unique indecomposable projective module $B_{k}[\mathrm{LO}, 5.6]$. Writing the $J(R)$-adic completion of $B_{k}$ as a direct sum of indecomposable modules yields a decomposition

$$
\hat{B}_{k} \cong \bigoplus_{i}\left(U_{k i}\right)^{s_{k i}}
$$

in which distinct ordered pairs $(k, i)$ yield non-isomorphic modules $U_{k i}$ and each $s_{k i}$ is the splitting number of $M_{k i}^{\prime}[\mathrm{LO}, 6.6]$.

Now we prove our corollary.

(i) $\Rightarrow$ (ii). We prove that condition (i) implies the equivalent form (2.8.1) of condition (ii). Since each projective $\Omega_{k}$-module $B_{k}$ is indecomposable and $\Omega$ is a splitting order, the $\Lambda_{Q}$-module $V=\left(B_{k}\right)_{Q}$ is simple [LO, 5.5]. By hypothesis (i), the $\hat{\Lambda}_{Q}$-module $V_{Q}=\left(\hat{B}_{k}\right)_{Q}$ is therefore indecomposable. Localizing decomposition (2.8.2) at $Q$ therefore shows that the right-hand side contains only one term. Since each $\left(U_{k i}\right)_{Q}$ is nonzero [LO, 6.11], (2.8.1) holds, as desired.

(ii) $\Rightarrow$ (i). Let $V$ be any simple $\Omega_{Q}$-module. By $[\mathrm{LO}, 5.5]$ we have $V \cong\left(B_{k}\right)_{Q}$ for some $k$. By our alternative form (2.8.1) of (ii), the right-hand side of (2.8.2) consists of the single term $U_{k i}$; and localizing this at $Q$ shows that $\hat{V}_{Q} \cong\left(U_{k 1}\right)_{Q}$. But by [LO, 6.11] $\left(U_{k 1}\right)_{Q}$ is an indecomposable projective $\hat{\Omega}_{Q}$-module. Since $\hat{\Lambda}_{Q}=\hat{\Omega}_{Q}$, condition (i) holds.

Supplementary Statement. It is proved in [LO, 6.16] that the present condition (ii), in its alternate form (2.8.1), implies KSM for $\Lambda$.

Next we show that Heller's condition can be refined to a necessary and sufficient condition for KSM.

Corollary 2.9. Suppose that $R$ is semilocal, $\Lambda$ is an indecomposable ring, and $\Lambda \neq \Gamma$. Then $\Lambda$ satisfies KSM if and only if:

(i) $Z(\Lambda)$ has exactly one singular maximal ideal $\mathbf{n}$ with respect to $\Lambda$; and 
(ii) For every simple $\left(\Lambda_{\mathbf{n}}\right)_{Q}$-module $V$, the (necessarily projective) $\left(\hat{\Lambda}_{\mathbf{n}}\right)_{Q}$-module $\hat{R}_{\mathbf{n}} \otimes_{R_{\mathbf{n}}} V$ is indecomposable.

Remarks 2.10 (on notation). The notation $\hat{R}_{\mathbf{n}}$ in (ii) can denote either the $J\left(R_{\mathbf{n}}\right)$-adic completion of $R_{\mathbf{n}}$ or, equivalently, the $\mathbf{n}$-localization of $\hat{R}$. Also, according to our standard convention the subscript $Q$ in (ii) denotes the $Q(R)$-localization, but this can be replaced by the $Q\left(R_{\mathbf{n}}\right)$-localization without changing the localization it yields [LO, 2.2].

Proof of 2.9. By our KSM theorem, condition (i) is necessary for KSM. Therefore, for the rest of this proof we assume that (i) holds and prove that KSM holds if and only if (ii) holds. Proposition 2.3 then states that $\Lambda$ satisfies KSM if and only if $\Lambda_{\mathbf{n}}$ does. Moreover, $Z(\Lambda)_{\mathbf{n}}=Z\left(\Lambda_{\mathbf{n}}\right)$. Therefore we may assume (after changing notation) that $\Lambda=\Lambda_{\mathbf{n}}$ and hence $Z(\Lambda)$ is local. Our corollary now follows from Corollary 2.8 and the KSM theorem.

On local endomorphism rings. Next we consider the question of whether KSM causes endomorphism rings to be local. As in several of our previous applications, we must avoid maximal orders, even locally. The notation $X \mid Y$ means that $X$ is isomorphic to a direct summand of $Y$.

Corollary 2.11. Suppose that $\Lambda$ is an indecomposable ring satisfying $K S M$, and that $\Lambda \neq \Gamma$. Let $\mathbf{n}$ be the singular maximal ideal of $Z(\Lambda)$ with respect to $\Lambda$. Then the endomorphism ring of the $\mathbf{n}$-localization of every indecomposable $\Lambda$-module is a local ring.

Proof. $\Lambda$ is not an artinian ring since $\Lambda \neq \Gamma$. Let $M$ be an indecomposable $\Lambda$ module. Our first objective is to prove that $M_{\mathbf{n}}$ is an indecomposable $\Lambda_{\mathbf{n}}$-module.

For the following argument recall the notational Remarks 2.10 above. Let $X$ be a nonzero direct summand of $M_{\mathbf{n}}$, and let $N$ be the $\Lambda$-submodule of $X$ generated by the numerators of some finite set of generators of the $\Lambda_{\mathbf{n}}$-module $X$. Then, by the Remarks above, $N_{\mathbf{n}}=X$ and $N_{Q}=X_{Q}$; and hence $X \mid M_{\mathbf{n}}$ yields $N_{Q} \mid\left(M_{\mathbf{n}}\right)_{Q}$. By [LO, Lemma 2.2], $\left(M_{\mathbf{n}}\right)_{Q} \mid M_{Q}$; hence $N_{Q} \mid M_{Q}$. We claim that $N_{\mathbf{m}} \mid M_{\mathbf{m}}$ for every maximal ideal $\mathbf{m}$ of $R$. This is true if $\mathbf{m}=\mathbf{n}$, because $N_{\mathbf{n}}=X$. So let $\mathbf{m}$ be any other maximal ideal. Since $\Lambda_{\mathbf{m}}=\Gamma_{\mathbf{m}}, \Lambda_{\mathbf{m}}$ is a maximal $R_{\mathbf{m}}$-order. Since $N_{Q} \mid M_{Q}$, we therefore have $N_{\mathbf{m}} \mid M_{\mathbf{m}}$ [LO, (4.1.8)]. Since $N_{\mathbf{m}} \mid M_{\mathbf{m}}$ for all maximal ideals $\mathbf{m}$, there is a $\Lambda$-module $N^{\prime}$ in the genus of $N$ such that $N^{\prime} \mid M$ [GL '89, 4.1]. Since $M$ is indecomposable and $N^{\prime} \neq 0$ we have $N^{\prime} \cong M$. Localizing at $\mathbf{n}$ then yields $X \cong M_{\mathbf{n}}$. Since a noetherian module cannot have a proper direct summand isomorphic to itself, $M_{\mathbf{n}}$ is indecomposable, as desired.

By Proposition 2.3, KSM passes from $\Lambda$ to $\Lambda_{\mathbf{n}}$. Note that $Z\left(\Lambda_{\mathbf{n}}\right)$ is isomorphic to $Z(\Lambda)_{\mathbf{n}}$, and is therefore a local ring. Therefore we can apply Corollary 2.5 to conclude that the $J\left(R_{\mathbf{n}}\right)$-adic completion $\left(M_{\mathbf{n}}\right)^{\wedge}$ is an indecomposable $\left(\Lambda_{\mathbf{n}}\right)^{\wedge}$ module.

Let $E(M)$ be the endomorphism ring of $M$. We have $E\left(M_{\mathbf{n}}\right)=E(M)_{\mathbf{n}}$. Therefore we may assume that $R$ is local (i.e. $R=R_{\mathbf{n}}$ ). We now wish to show that $E(M)$ is a local ring.

By completeness of $\hat{\Lambda}$ and indecomposability of $\hat{M}$, the $\hat{\Lambda}$-endomorphism ring $E(\hat{M})$ is a local ring [R 75 , p. 88, Exercise 5].

The ring $E=E(M)$ is a module-finite $R$-algebra, and $\hat{E}=E(M)^{\wedge}=E(\hat{M})$, which is a local ring. To conclude that $E$ itself is local, it suffices to recall [LO, 
$3.8]$ that the set of left ideals $X$ of $\hat{E}$ such that $\hat{E} / X$ has finite length as an $\hat{E}$ module corresponds bijectively with the set of left ideals $N$ of $E$ such that $E / N$ has finite length as an $E$-module, via contraction and extension, and the composition length of $\hat{E} / X$ as a $\hat{E}$-module equals the composition length of $E /(E \cap X)$ as an E-module.

Remarks 2.12 (on TFKS). (i) Examples of rings of types (c2) and (c3) in the TFKS theorem, when $R$ is semilocal. Since $R$ is semilocal we can ignore condition (a), and so the only difference between TFKS and KSM is the presence of the quite small classes of rings $\Lambda$ that satisfy conditions (c2) or (c3). Class (c2) contains both commutative and noncommutative rings. Commutative examples are easily constructed by taking $\Gamma$ to be any semilocal principal ideal domain with two maximal ideals $M, N$ that have the same residue field $F$. Then let $f, g$ be surjections: $\Gamma \rightarrow F$ with kernels $M, N$ respectively and let $\Lambda=\mathrm{eq}(\Gamma, f, g)$. In particular, there are rings of type (c2) that are integral domains whose field of quotients has dimension 2 over the rational numbers.

Class (c3) contains only noncommutative rings. We give examples at the end of $\S 11$.

(ii) Perhaps the most surprising fact about conditions (c2) and (c3) is that the exceptional rings cannot occur when $\Gamma$ is not module-finite over $R$. In other words, unless $\Gamma$ is module-finite over $R$, the only difference between TFKS and KSM lies in condition (a).

(iii) Structure of $\Lambda_{\mathbf{n}}$ in situations (c2) and (c3). $\Lambda_{\mathbf{n}}$ is matrix-local. In fact, $\Lambda_{\mathbf{n}}^{\prime}$ is local. See $\S \S 9-11$ for this and other detailed properties of $\Lambda_{\mathbf{n}}$.

(iv) In order for $\Lambda$ to satisfy TFKS it is not necessary for indecomposable torsionfree $\Lambda$-modules or $\Lambda_{Q}$-modules to have indecomposable completions, even if we exclude maximal orders both locally and globally. This contrasts with the analogous KSM situations 2.5, 2.7, and 2.9. In fact, both exceptional classes (c2) and (c3) yield counterexamples.

For the simplest counterexamples, consider any $\Lambda$ of type (c2) or (c3) that is an integral domain (in which case $\Gamma$ is also an integral domain and, in the notation of our TFKS theorem, we have $\Lambda=\Lambda^{\prime}$ and $\Gamma=\Gamma^{\prime}$ ). After localizing at the unique singular maximal ideal $\mathbf{n}$ of $Z(\Lambda)$ and changing notation, we have that $Z(\Lambda)$ is a local ring with maximal ideal $\mathbf{n}$. Since $\Lambda$ and $\Gamma$ are integral domains with quotient division ring $\Lambda_{Q}=\Gamma_{Q}$, both $\Lambda$ and $\Gamma$ are indecomposable $\Lambda$-modules. However, in both cases (c2) and (c3), the $J(R)$-adic completion $\hat{\Gamma}$ fails to be an indecomposable $\hat{\Lambda}$-module because $\Gamma$ maps onto a ring with nontrivial idempotents, namely $\Gamma /(M \cap N)$ in situation (c2), and $\Gamma / M$ in situation (c3). This yields the desired counterexamples to the torsionfree analogs of 2.5 and 2.7 .

For a counterexample to the torsionfree analog of 2.9 , consider the same rings $\Lambda$ as in the previous paragraph. Since $\Gamma$ is an integral domain, the unique simple $\Gamma_{Q}$-module is $\Gamma_{Q}$ itself. But since $\hat{\Gamma}$ decomposes, so does $\hat{\Gamma}_{Q}=\hat{R} \otimes \Gamma_{Q}$.

(v) The TFKS analog of Corollary 2.11, about local endomorphism rings, fails for the exceptional rings of types (c2) and (c3). Let $\Lambda$ be as in part (iv) of these remarks. In particular, $\Lambda$ is an integral domain whose local center has maximal ideal $\mathbf{n}$ singular with respect to $\Lambda$, and $\Lambda$ satisfies TFKS.

In this situation, $\Gamma$ is an indecomposable $\Lambda$-module; and its endomorphism ring as a $\Lambda$-module is the ring $\Gamma$ since $\Lambda_{Q}=\Gamma_{Q}$. The $\mathbf{n}$-localization of $\Gamma$ again equals $\Gamma$ 
since $Z(\Lambda)$ is local. On the other hand, $\Gamma$ is not a local ring because, as explained in part (iv), $\Gamma / J(\Gamma)$ has nontrivial idempotents.

\section{Semilocal Reduction, Semilocal Notation}

In this section we reduce the proofs of our two main theorems (KSM and TFKS) to the situation that $R$, and hence $Z(\Lambda)$, are semilocal rings. At the end of the section we collect, for easy reference, the standard notation used in the rest of the paper, when $R$ is semilocal. The notation $\mathcal{M}$ always denotes a finite set of maximal ideals of $R$, and $R_{\mathcal{M}}, \Lambda_{\mathcal{M}}, \ldots$ denotes the localization that inverts the multiplicatively closed set $R-\bigcup \mathcal{M}$.

The steps of our reduction are the same for KSM as for TFKS. First we show that KSM holds for $\Lambda$ if and only if every genus consists of a single isomorphism class and KSM holds for genera of modules [Lemma 3.1]. Second, we show that KSM holds for genera of $\Lambda$-modules if and only if the localization $\Lambda_{\mathcal{M}}$ satisfies KSM for every finite set $\mathcal{M}$ of maximal ideals of $Z(\Lambda)$ [Lemma 3.2]. The analogous statements hold for TFKS, and the two parts are put together in Lemma 3.3.

In the process we show that conditions (b) and (c) of our KSM theorem are equivalent to the statement that KS holds for genera of $\Lambda$-modules. Again the analogous statement holds for TFKS.

All this is considerably simpler when $\Lambda$ is contained in a maximal $R$-order in $\Lambda_{Q}$. For, we can then let $\mathcal{M}$ be the finite set of maximal ideals $\mathbf{m}$ of $Z(\Lambda)$ such that $\Lambda_{\mathbf{m}} \neq \Gamma_{\mathbf{m}}$; and then we can use the well-known fact that KSM (resp. TFKS) holds for genera of $\Lambda$-modules if and only it holds for $\Lambda_{\mathcal{M}}$-modules.

Returning to our general case, we say that $\Lambda$ satisfies KSM for genera of modules if whenever

$$
M_{1} \oplus M_{2} \oplus \ldots \oplus M_{m} \cong N_{1} \oplus N_{2} \oplus \ldots \oplus N_{n}
$$

with each $M_{i}, N_{j}$ an indecomposable, (f.g. left) $\Lambda$-module, then we must have $m=n$ and, after a suitable renumbering of the $N_{i}$, every genus $\left(M_{i}\right)=\operatorname{genus}\left(N_{i}\right)$. An analogous definition, in which every $M_{i}$ and $N_{j}$ is torsionfree, applies to TFKS for genera of modules.

The following facts about an arbitrary $\Lambda$-module $M$ show that these are really properties of genera of $\Lambda$-modules. (i) If $M$ is indecomposable, then so is every module in genus( $M)$ [GL '89, 4.2]. (ii) If $M=\bigoplus_{i} M_{i}$ and $N \in \operatorname{genus}(M)$, then $N=\bigoplus_{i} N_{i}$ with every $N_{i} \in \operatorname{genus}\left(M_{i}\right)$ [GL '89, 4.3]. (iii) If $M$ is torsionfree, then so is every module in genus $(M)$. (Since $R$ has no nilpotent elements, its set of non-zero-divisors is $R-\bigcup Q$; and this localizes properly, as shown in [LO, 2.3].)

Lemma 3.1. $\Lambda$ satisfies KSM (respectively TFKS) if and only if

(a) Every genus of $\Lambda$-modules (resp. torsionfree modules) consists of a single isomorphism class; and

(b) $\Lambda$ satisfies KSM (respectively TFKS) for genera of modules.

Proof. Necessity of condition (a). Let $M$ be any $\Lambda$-module, and suppose that $N \in \operatorname{genus}(M)$ but $N \neq M$. Then there is a module $X$, necessarily in genus $(M)$, such that

$$
M \oplus M \cong N \oplus X .
$$

This is a consequence of a version of Serre's direct-summand theorem, in dimension 1 [GL '88, Lemma 1.2]. If $M$ is indecomposable, then so are $N$ and $X$, as noted 
just before the statement of our lemma, so KS fails. Thus it suffices to show that, if (a) fails, then it fails for some indecomposable $M$. So suppose that $\Lambda$-modules $M, N$ are in the same genus, and choose a decomposition $M=\bigoplus_{i} M_{i}$ with each $M_{i}$ indecomposable. Then, as noted just before the statement of our lemma, there is a decomposition $N=\bigoplus_{i} N_{i}$ with each $N_{i} \in \operatorname{genus}\left(M_{i}\right)$. If the genus of every indecomposable $\Lambda$-module consists of a single isomorphism class, then we have every $M_{i} \cong N_{i}$, and hence $M \cong N$. This completes the proof that condition (a) is necessary for KS.

Necessity of condition (b) is obvious, as is sufficiency of the combination of conditions (a) and (b).

Lemma 3.2. $\Lambda$ satisfies KSM for genera of modules if and only if, for every finite set $\mathcal{M}$ of maximal ideals of $R, \Lambda_{\mathcal{M}}$ satisfies KSM. The analogous statement about TFKS holds too.

Proof. We deal only with KSM, since the proof for TFKS is exactly the same. Suppose that KS fails for genera of $\Lambda$-modules, and let

$$
\bigoplus_{i=1}^{m} M_{i} \in \operatorname{genus}\left(\bigoplus_{i=1}^{n} N_{i}\right)
$$

be a counterexample; that is, each $M_{i}, N_{i}$ is an indecomposable $\Lambda$-module and there is no way of renumbering the $N_{i}$ so that, for all maximal ideals $\mathbf{m}$ of $R$ and all $i,\left(M_{i}\right)_{\mathbf{m}} \cong\left(N_{i}\right)_{\mathbf{m}}$. (This includes the possibility that $m \neq n$.) We now build a finite set $\mathcal{M}$ of maximal ideals of $R$ such that localizing (3.2.1) at $\mathcal{M}$ yields a counterexample to KSM for $\Lambda_{\mathcal{M}}$.

Consider any renumbering of the $N_{i}$. Then, since (3.2.1) is a counterexample to KS for genera of $\Lambda$-modules, there is a maximal ideal $\mathbf{m}$ of $R$ such that, for some $i,\left(M_{i}\right)_{\mathbf{m}} \neq\left(N_{i}\right)_{\mathbf{m}}$ (perhaps because $m \neq n$ and there is no $M_{i}$ or $N_{i}$ for that $i$ ). There are only finitely many renumberings of the $N_{i}$. For each renumbering, include one such $\mathbf{m}$ in $\mathcal{M}$. Then, even if $\mathcal{M}$ is enlarged, there is no renumbering of the $N_{i}$ such that, for every $\mathbf{m} \in \mathcal{M},\left(M_{i}\right)_{\mathbf{m}} \cong\left(N_{i}\right)_{\mathbf{m}}$ for all $i$.

If we can enlarge $\mathcal{M}$ so that every $\left(M_{i}\right)_{\mathcal{M}}$ and $\left(N_{i}\right)_{\mathcal{M}}$ is an indecomposable $\Lambda_{\mathcal{M}}$-module, we will have a counterexample to KSM for $\Lambda_{\mathcal{M}}$ (because "genus" and "isomorphism class" are the same thing when $R$ is semilocal). It is proved in [LO, 2.20 ] that, for any indecomposable $\Lambda$-module $X$, there is a finite set $G$ of maximal ideals of $R$ such that $X_{G}$ is $\Lambda_{G}$-indecomposable, and remains indecomposable even if $G$ is enlarged. A finite number of enlargements of $\mathcal{M}$ - one for every $M_{i}$ and

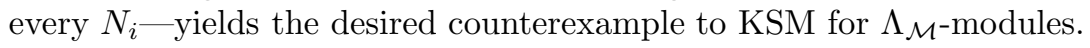

Conversely, suppose that KSM fails for some $\Lambda_{\mathcal{M}}$, and choose a particular counterexample:

$$
\bigoplus_{i=1}^{m} X_{i} \cong \bigoplus_{i=1}^{n} Y_{i}
$$

We use this to construct a counterexample (3.2.1) to KS for genera of $\Lambda$-modules. As a first approximation, let $M_{i}$ and $N_{i}$ be the $\Lambda$-modules generated by some finite set of $\Lambda_{\mathcal{M}}$-generators of $X_{i}$ and $Y_{i}$ respectively. Then we have

$$
\left(M_{i}\right)_{\mathcal{M}} \cong\left(X_{i}\right) \text { and }\left(M_{i}\right)_{Q} \cong\left(X_{i}\right)_{Q} \cong\left(\left(M_{i}\right)_{\mathcal{M}}\right)_{Q}
$$

together with the analogous assertions for $N$. Before proceeding we prove the isomorphism $\left(M_{i}\right)_{Q} \cong\left(\left(M_{i}\right)_{\mathcal{M}}\right)_{Q}$ in (3.2.3). Consider the natural map $\nu$ from the 
left-hand side to the right-hand side. By $[\mathrm{LO}, 2.2], \nu$ is a surjection. To see that $\nu$ is one-to-one, first note that, by construction, $M_{i} \subseteq\left(M_{i}\right)_{\mathcal{M}}=X_{i}$. Tensoring this inclusion with the flat $R$-module $R_{Q}$ therefore shows that $\nu$ is one-to-one, as desired.

Because of the first isomorphism in (3.2.3), and the fact that $R_{\mathcal{M}}$ is semilocal, there is no way to renumber the $N_{i}$ such that, for all $i$ and all $\mathbf{m} \in \mathcal{M},\left(M_{i}\right)_{\mathbf{m}} \cong$ $\left(N_{i}\right)_{\mathbf{m}}$. Therefore, if (3.2.1) holds and each $M_{i}$ and $N_{i}$ is indecomposable, we have our desired counterexample to KSM for genera of $\Lambda$-modules. Before proceeding further, we claim that

$$
(\forall \mathbf{n} \notin \mathcal{M})(\forall i)\left(M_{i}\right)_{\mathbf{n}} \text { and }\left(N_{i}\right)_{\mathbf{n}} \text { are } Q \text {-torsionfree } R \text {-modules. }
$$

Let $\mathbf{n}$ be given; write $M=M_{i}$ and $X=X_{i}$; also, let $x \in M$ be such that $(x / 1)$ is a $Q\left(R_{\mathbf{n}}\right)$-torsion element of $M_{\mathbf{n}}$ (hence of $\left.X_{\mathbf{n}}\right)$. So $x / 1=0$ in $\left(X_{\mathbf{n}}\right)_{Q\left(R_{\mathbf{n}}\right)}=$ $\left(X_{\mathbf{n}}\right)_{Q(R)}$. We want to show that $x / 1=0$ in $X_{\mathbf{n}}$ (hence in $M_{\mathbf{n}}$ ). It suffices to find $d \in R-\mathbf{n}$ such that $d x=0$.

Since $x / 1=0$ in $\left(X_{\mathbf{n}}\right)_{Q(R)}$ we have $a b x=0$ for some $a \in R-\mathbf{n}$ and $b \in$ $R-\bigcup Q$. Then $R / R b$ is an artinian ring, and hence so is $(R / R b)_{\mathcal{M}}=R_{\mathcal{M}} /(R b)_{\mathcal{M}}$. Therefore the Jacobson radical of this last ring is nilpotent. This implies that $J\left(R_{\mathcal{M}}\right)^{n} \subseteq(R b)_{\mathcal{M}}$ for some $n$.

By the Chinese Remainder Theorem there is an element $c \in R$ such that $c$ belongs to every maximal ideal in $\mathcal{M}$ and $c \equiv 1(\bmod \mathbf{n})$. We set $d=a c^{n} \in R-\mathbf{n}$.

Now $(c / 1) \in J\left(R_{\mathcal{M}}\right)$ implies $R_{\mathcal{M}}(c / 1)^{n} \subseteq(R b)_{\mathcal{M}}$. Since $X$ is an $R_{\mathcal{M}}$-module we have $c x=(c / 1) x$ - where $c / 1 \in R_{\mathcal{M}}$ - and therefore $a c^{n} x=(a / 1)(c / 1)^{n} x \subseteq$ $R_{\mathcal{M}}(a / 1)(b / 1) x=0$, completing the proof of (3.2.4).

Consider (3.2.1). We wish to have $\left(\bigoplus_{i} M_{i}\right)_{\mathbf{m}} \cong\left(\bigoplus_{i} N_{i}\right)_{\mathbf{m}}$ for all maximal ideals $\mathbf{m}$. We make use of package deal results about localizations to change the $\mathbf{m}$-localization $\left(M_{i}\right)_{\mathbf{m}}$ and $\left(N_{i}\right)_{\mathbf{m}}$ for all $\mathbf{m}$ such that the desired isomorphism does not already hold. Note that no change is required if $\mathbf{m} \in \mathcal{M}$, because of (3.2.2).

In view of (3.2.3) and its analog for $N$ we have

$$
\bigoplus_{i}\left(M_{i}\right)_{Q} \cong \bigoplus_{i}\left(N_{i}\right)_{Q}
$$

Therefore, by [LO, Lemma $2.4(\mathrm{ii})],\left(\bigoplus_{i} M_{i}\right)_{\mathbf{m}} \cong\left(\bigoplus_{i} N_{i}\right)_{\mathbf{m}}$ for all but finitely many $\mathbf{m}$. Let $\mathbf{n}$ be one of the finitely many maximal ideals of $R$ such that $\left(\bigoplus_{i} M_{i}\right)_{\mathbf{n}} \neq$ $\left(\bigoplus_{i} N_{i}\right)_{\mathbf{n}}$. As already noted, $\mathbf{n} \notin \mathcal{M}$.

According to the package deal theorem for localizations of modules [LO, Theorem 2.9], we can find (for each $i$ ) a new module $M_{i}$ such that $\left(M_{i}\right)_{\mathbf{n}}$ is isomorphic to any prescribed $\Lambda_{\mathbf{n}}$-module whose $Q$-localization is isomorphic to the original $\left(\left(M_{i}\right)_{\mathbf{n}}\right)_{Q}$, and whose localizations at maximal ideals $\neq \mathbf{n}$ are isomorphic to those of the original $M_{i}$.

Return to (3.2.5). Express each $\left(M_{i}\right)_{Q}$ as a direct sum of indecomposable modules over the artinian ring $\Lambda_{Q}$, and let $W_{1}, W_{2}, \ldots$ be the distinct non-isomorphic modules that result in this way. This gives (for each $i$ ) an expression $\left(M_{i}\right)_{Q} \cong$ $\bigoplus_{j}\left(W_{j}\right)^{b_{i j}}$. Since KSM holds for artinian rings, we get the same set of modules $W_{j}$ by working with the $\left(N_{i}\right)_{Q}$, and therefore an expression of the form $\left(N_{i}\right)_{Q} \cong \bigoplus_{j}\left(W_{j}\right)^{c_{i j}}$. Then KSM for $\Lambda_{Q}$ together with (3.2.5) yields

$$
\sum_{i} b_{i j}=\sum_{i} c_{i j}
$$


for each $j$. For each $W_{j}$ choose a (necessarily $R$-torsionfree) $\Lambda_{\mathbf{n}}$-submodule $V_{j}$ of $W_{j}$ such that $\left(V_{j}\right)_{Q} \cong W_{j}$. Replace each $\left(M_{i}\right)_{\mathbf{n}}$ by $\bigoplus_{j}\left(V_{j}\right)^{b_{i j}}$ and each $\left(N_{i}\right)_{\mathbf{n}}$ by $\bigoplus_{j}\left(V_{j}\right)^{c_{i j}}$. Then $(3.2 .6)$ shows that $\left(\bigoplus_{i} M_{i}\right)_{\mathbf{n}} \cong\left(\bigoplus_{i} N_{i}\right)_{\mathbf{n}}$, and our replacement is complete. After a finite number of repetitions of this procedure, (3.2.1) holds.

All that remains to be done now is to show that every $M_{i}$ and $N_{i}$ is indecomposable. So choose some $M_{i}$ and suppose that $M_{i}=A \oplus B$. Since $\left(M_{i}\right)_{\mathcal{M}} \cong X_{i}$, which is $\Lambda_{\mathcal{M}}$-indecomposable, we must have, say, $B_{\mathcal{M}}=0$. To show that $B=0$ it suffices to show that $B_{\mathbf{n}}=0$ when $\mathbf{n} \notin \mathcal{M}$.

Since $B_{\mathbf{n}}$ is $Q$-torsionfree, by (3.2.4), it suffices to show that $\left(B_{\mathbf{n}}\right)_{Q}=0$. It therefore suffices to show that $B_{Q}=0$. This is equivalent to the assertion that $B_{\mathbf{p}}=0$ for all minimal prime ideals $\mathbf{p}$ [LO, 2.2(i)]. If $\mathbf{p}$ is contained in some element of $\mathcal{M}$ we have $B_{\mathbf{p}}=\left(B_{\mathcal{M}}\right)_{\mathbf{p}}$, which equals zero since $B_{\mathcal{M}}=0$. Suppose therefore that $\mathbf{p}$ is not contained in any element of $\mathcal{M}$. Since $B \subseteq M_{i}$ it suffices to check that $\left(M_{i}\right)_{\mathbf{p}}=0$. But localizing $(3.2 .3)$ at $\mathbf{p}$ yields $\left.\left(M_{i}\right)_{\mathbf{p}} \cong\left(\left(M_{i}\right)_{\mathcal{M}}\right)\right)_{\mathbf{p}}$, which equals zero since $\mathbf{p}$ is contained in no element of $\mathcal{M}$ [LO, 2.2(ii)].

Lemma 3.3. If Theorems 1.1 (KSM) and 1.3 (TFKS) hold whenever $R$ is semilocal, then they hold for all $R$.

Proof. We may assume that $R=Z(\Lambda)$. Moreover, we are assuming that $\Lambda$ is an indecomposable, non-artinian ring. Our proof uses the fact that normalizations localize [LO, 4.3] without explicit mention.

Claim 1. There is a finite set $\mathcal{M}$ of maximal ideals of $R$ such that $\Lambda_{\mathcal{M}}$ is an indecomposable ring, and remains indecomposable if $\mathcal{M}$ is enlarged. (Thus we have indecomposable rings $\Lambda_{\mathcal{M}}$ to apply the semilocal case of the theorem to!) Note that $\Lambda$ is an indecomposable ring if and only if its center is (because ring decompositions arise from orthogonal central idempotents). Since $\Lambda$ is indecomposable by hypothesis, so is $Z(\Lambda)$. By [LO, Theorem 2.20], with $R=Z(\Lambda)=\Lambda$ we find a finite set $\mathcal{M}$ of maximal ideals such that $Z(\Lambda)_{\mathcal{M}}$ is indecomposable and remains so if $\mathcal{M}$ is enlarged. Since $Z(\Lambda)_{\mathcal{M}}=Z\left(\Lambda_{\mathcal{M}}\right)$ we see that $\Lambda_{\mathcal{M}}$ is indecomposable, as required.

Claim 2. The indecomposable ring $\Lambda_{\mathcal{M}}$ resulting from Claim 1 is not artinian. It is equivalent to prove that the indecomposable ring $R_{\mathcal{M}}=Z\left(\Lambda_{\mathcal{M}}\right)$ is not artinian. Since $R$ is indecomposable and non-artinian, no maximal ideal of $R$ is a minimal prime [LO, 1.3]. Therefore no maximal ideal of $R_{\mathcal{M}}$ is a minimal prime, and therefore $R_{\mathcal{M}}$ is not artinian.

Suppose, now, that $\Lambda$ satisfies KSM. Then condition (a) of the KSM theorem holds, by Lemma 3.1. We need to verify conditions (b) and (c).

Condition (b). Choose $\mathcal{M}$ as in Claim 1. Then the indecomposable, non-artinian ring $\Lambda_{\mathcal{M}}$ satisfies KSM by Lemmas 3.1 and 3.2. Let $\mathbf{n}$ be any maximal ideal of $R$ that is singular with respect to $\Lambda$, and let $\mathbf{m}$ be any other maximal ideal of $R$. To see that $\mathbf{m}$ is not also singular, enlarge $\mathcal{M}$, if necessary, so that $\mathbf{m}, \mathbf{n} \in \mathcal{M}$. Since $\Lambda_{\mathcal{M}}$ satisfies KSM, the semilocal version of the KSM theorem implies there is at most one singular maximal ideal in $\mathcal{M}$. Since $\mathbf{n}$ is singular, $\mathbf{m}$ cannot be.

Condition (c). As in the proof of condition (b), choose $\mathcal{M}$ such that $\mathbf{n} \in \mathcal{M}$, and recall that $\Lambda_{\mathcal{M}}$ satisfies KSM. Since $\Lambda_{\mathbf{n}}=\left(\Lambda_{\mathcal{M}}\right)_{\mathbf{n}}$, condition (c) follows from the semilocal version of the KSM theorem.

Now consider TFKS. Conditions (a) and (b) follow as above. Therefore, to complete the proof we can suppose that the singular maximal ideal $\mathbf{n}$ exists and $\Lambda_{\mathbf{n}}$ 
does not satisfy condition (c1). We need to show that it satisfies (c2) or (c3). As above, we can suppose that $\mathbf{n} \in \mathcal{M}$ and the semilocal ring $\Lambda_{\mathcal{M}}$ is indecomposable and non-artinian. Therefore, by the semilocal version of the TFKS theorem, $\Lambda_{\mathcal{M}}$ has the form (c2) or (c3).

Claim 3. $\Gamma$ is module-finite over $R$. By the semilocal version of the theorem $\Gamma_{\mathcal{M}}$ is module-finite over $R_{\mathcal{M}}$. Let the set $\left\{\gamma_{i}\right\}$ be the numerators of some finite set of generators of the $R_{\mathcal{M}}$-module $\Gamma_{\mathcal{M}}$ together with some finite set of generators of the $R$-module $\Lambda$. Since $\Gamma_{\mathbf{m}}=\Lambda_{\mathbf{m}}$ for every $\mathbf{m} \notin \mathcal{M}$ (in fact, for every $\mathbf{m} \neq \mathbf{n}$ ), we see that $\left\{\gamma_{i}\right\}$ is a finite set of generators of the $R$-module $\Gamma$.

Claim 4. $\Lambda \cong P^{n}$ for some projective $\Lambda$-module $P$ such that $P_{Q}$ is a simple $\Lambda_{Q^{-}}$ module, and some $n$. By the semilocal version of the theorem, $\Lambda_{\mathcal{M}}$ has such a decomposition. Therefore, by further localization, $\Lambda_{\mathbf{n}} \cong X^{n}$ where $X_{Q}$ is a simple $\Lambda_{Q}$-module. Let $P$ be the $\Lambda$-module generated by the numerators of some finite set of $\Lambda_{\mathbf{n}}$-generators of $X$. Then $P_{\mathbf{n}}=X$, and $P_{Q}=X_{Q}$ since $X_{Q}$ is simple. We show that $P$ works.

Since $\Lambda_{\mathbf{m}}=\Gamma_{\mathbf{m}}$ for every $\mathbf{m} \neq \mathbf{n}$, and $\Gamma$ is a maximal order, the fact that $\Lambda_{Q} \cong\left(X_{Q}\right)^{n}=\left(P_{Q}\right)^{n}$ shows that $\Lambda_{\mathbf{m}} \cong\left(P_{\mathbf{m}}\right)^{n}$ whenever $\mathbf{m} \neq \mathbf{n}$. Therefore $\Lambda$ is in the same genus as $P^{n}$. Since $\Lambda$ satisfies TFKS, every genus of torsionfree $\Lambda$-modules consists of a single isomorphism class [Lemma 3.1] and therefore $\Lambda \cong P^{n}$, proving the claim.

Claim 5. $\Lambda=M_{n}\left(\Lambda^{\prime}\right)$ and $\Gamma=M_{n}\left(\Gamma^{\prime}\right)$ where $\Lambda^{\prime} \subset \Gamma^{\prime}$ and $\Gamma^{\prime}$ is a PID. Let $\Lambda^{\prime}=E(P)$ and $\Gamma^{\prime}=E(\Gamma P)$ where $E(\ldots)$ denotes " $\Lambda$-endomorphism ring of ...". Note that $E(\Gamma P)$ is also the $\Gamma$-endomorphism ring of $\Gamma P$ since both $\Gamma$ and $P$ are contained in their $Q$-localizations. Claim 4 yields $\Lambda=M_{n}\left(\Lambda^{\prime}\right)$ and $\Gamma=M_{n}\left(\Gamma^{\prime}\right)$, and $\Lambda^{\prime} \subset \Gamma^{\prime}$. It remains to show that $\Gamma^{\prime}$ is a PID. Since $P_{Q}$ is a simple $\Lambda_{Q}$-module, $\Gamma_{Q}^{\prime}$ is a division ring, and hence $\Gamma^{\prime}$ is an integral domain. Since $\Gamma$ is a maximal $R$-order in $\Gamma_{Q}, \Gamma^{\prime}$ is a maximal $R$-order in $\left(\Gamma^{\prime}\right)_{Q}$. If $\Gamma^{\prime}$ were not a PID, it would therefore not satisfy KS for projective modules. Since $\Gamma$ is Morita equivalent to $\Gamma^{\prime}$, $\Gamma$ would likewise not satisfy KS for projective modules. But TFKS for $\Lambda$ implies TFKS for $\Gamma$, by the proof of Corollary 2.4(b). [Note. In Corollary $2.4 R$ is semilocal, but the proof of part (b) does not use this fact.] This contradiction completes the proof of Claim 5.

This completes the proof that $\Lambda$ has the properties that are common to $(\mathrm{c} 2)$ and (c3). We now suppose that $\Lambda_{\mathcal{M}}^{\prime}$ has the form (c2), and prove that $\Lambda^{\prime}$ likewise has this form. We have $\left(\Lambda^{\prime}\right)_{\mathcal{M}}=\mathrm{eq}\left(\left(\Gamma^{\prime}\right)_{\mathcal{M}}, f, g\right)$, where $f$ and $g$ are surjective ring homomorphisms: $\left(\Gamma^{\prime}\right)_{\mathcal{M}} \rightarrow \Psi$ where $\operatorname{ker}(f) \neq \operatorname{ker}(g)$ and $\Psi$ is a local ring that has finite length as an $R_{\mathcal{M}}$-module, and hence every $R$-submodule of $\Psi$ is an $R_{\mathcal{M}}$-module. Therefore $f$ and $g$ map $\Gamma^{\prime}$ itself onto $\Psi$. Let $E=\operatorname{eq}\left(\Gamma^{\prime}, f, g\right)$. Then we have $\Lambda_{\mathcal{M}}^{\prime}=E_{\mathcal{M}}$ and $\Lambda_{\mathbf{m}}^{\prime}=\Gamma_{\mathbf{m}}^{\prime}=E_{\mathbf{m}}$ for all $\mathbf{m} \notin \mathcal{M}$. It follows that $\Lambda^{\prime}=E$, as desired.

We omit the similar details for rings of the form (c3), and proceed to the proof of the converse part of the theorem. Thus we assume, now, that $\Lambda$ satisfies properties (a)-(c) of the KSM theorem or the TFKS theorem, and we want to prove that $\Lambda$ satisfies KSM or TFKS, respectively. This a relatively straightforward application of Lemmas 3.1 and 3.2, together with Claims 1 and 2 and the semilocal versions of the theorems we are proving, so we omit the details. 
The proofs of the preceding three lemmas contain the proof of the following result, which might be of some interest in its own right.

Proposition 3.4. $\Lambda$ satisfies KSM (resp. TFKS) for genera of modules if and only if conditions (b) and (c) of the KSM Theorem 1.1 (resp. TFKS Theorem 1.3) hold.

Notation 3.5 (Semilocal). For the rest of this paper, unless the contrary is explicitly stated, the commutative ring $R$ is semilocal, (noetherian, of Krull dimension 1,) with no nilpotent elements. $J(S)$ denotes the Jacobson radical of any ring $S$. The permanent notation in 2.1 remains in force. In particular, $\Lambda$ denotes an $R$-order in the semisimple artinian ring $\Lambda_{Q}$ and $\Gamma=\bigoplus_{k} \Gamma_{k}$ always denotes a normalization of $\Lambda$, decomposed as in (2.1.2).

If a ring $A$ is isomorphic to a full $n \times n$ matrix over a noetherian integral domain (perhaps over a division ring), we say that $A$ has matrix size $n$. This integer $n$ is unique because of the hypotheses on $A$. Since $R$ is semilocal, each $\Gamma_{k}$ is a full matrix ring over a left and right principal ideal domain [LO, (4.1.8)]. We usually denote its matrix size by $n_{k}$. Since $\Gamma_{k}$ is a full matrix ring over a principal ideal domain that is an $R$-algebra (not necessarily module-finite over $R$ ), we can introduce the following notation. See [LO, 6.1] for more details.

Simple modules $S_{k i}$ and maximal ideals $M_{k i}^{\prime}$. Each $\Gamma_{k}$ has only finitely many nonisomorphic simple (left) modules $S_{k i}$. For each $(k, i)$ we let $M_{k i}^{\prime}$ denote the annihilator in $\Gamma_{k}$ of $S_{k i}$. Then $\Gamma_{k} / M_{k i}^{\prime}$ is a simple artinian ring and the indexed set $\left\{M_{k i}^{\prime}\right\}$ is the set of all maximal (2-sided) ideals of $\Gamma_{k}$.

Splitting numbers $s_{k i}$. Since $\Gamma_{k}$ is a full matrix ring over a principal ideal domain, it has a unique (up to isomorphism) indecomposable f.g. projective left module $\Gamma_{k} e_{k}$, where $e_{k}$ is an arbitrary primitive idempotent of $\Gamma_{k}$. Since the ring $\Gamma_{k} / M_{k i}^{\prime}$ is simple artinian and its simple module is $S_{k i}$, we have a decomposition

$$
\Gamma_{k} e_{k} / M_{k i}^{\prime} e_{k} \cong\left(S_{k i}\right)^{s_{k i}}, \quad \text { where } \quad M_{k i}^{\prime}=\operatorname{ann}_{\Gamma_{k}} S_{k i} .
$$

We call the multiplicity $s_{k i}$ the splitting number of $M_{k i}^{\prime}$, or the $(k, i)$-splitting number of $\Gamma$. We have

(3.5.2) Let $s=s_{k i}$ and $M^{\prime}=M_{k i}^{\prime}$ be as in (3.5.1). Then:

(i) Every primitive idempotent in $\Gamma_{k}$ splits into the sum of $s$ primitive orthogonal idempotents in $\Gamma_{k} / M^{\prime}$.

(ii) Let $\mathbf{n}=M^{\prime} \cap Z\left(\Gamma_{k}\right)$. Then $\mathbf{n}$ is the unique maximal ideal of $Z\left(\Gamma_{k}\right)$ contained in $M^{\prime}$, and every primitive idempotent in $\left(\Gamma_{k}\right)_{\mathbf{n}}$ splits into the sum of $s$ primitive orthogonal idempotents in $\left(\Gamma_{k}\right)_{\mathbf{n}} / M_{\mathbf{n}}^{\prime}=\Gamma_{k} / M^{\prime}$.

Statement (i) is a way of restating (3.5.1). The first assertion of (ii) follows from the fact that the maximal ideals of $\Gamma_{k}$ and $Z\left(\Gamma_{k}\right)$ correspond bijectively via contraction [LO, (4.1.6)]. The equality at the end of (ii) follows from the fact that $\Gamma_{k} / M^{\prime}$ has finite length as a $Z\left(\Gamma_{k}\right)$-module since $\Gamma_{k}$ is always a classical maximal order over its center $[\mathrm{LO},(4.1 .2)]$. For details, see the proof of the stronger statement [LO, (4.1.9)]. The statement about primitive idempotents follows from (i) and the fact that primitive idempotents in the maximal $Z\left(\Gamma_{k}\right)$-order $\Gamma_{k}$ remain primitive in its simple artinian quotient ring, hence in $\left(\Gamma_{k}\right)_{\mathbf{n}}$.

Although the splitting numbers $s_{k i}$ were defined using left modules, their values are the same as their right-handed counterparts. Moreover, they are independent of the particular normalization $\Gamma$ since any two normalizations of $\Lambda$ are conjugate in $\Lambda_{Q}$. See [LO, (6.1.6) and (4.1.7)]. 
The following statement, whose proof is given in [LO, 1.4], describes the type of ideal that we use for a conductor ideal.

(3.5.3) Let $\Omega$ be any $R$-order such that $\Lambda \subseteq \Omega \subseteq \Lambda_{Q}$, and assume that $Z(\Lambda)$ has no artinian ring-direct summands. Then $\Lambda$ and $\Omega$ have a common ideal $C$ such that

(i) $\Lambda / C$ and $\Omega / C$ are $R$-modules of finite length (hence artinian rings);

(ii) $C \subseteq J(\Lambda)$ (equivalently, $C \subseteq J(\Omega)$ ); and

(iii) $C_{Q}=\Lambda_{Q}\left(=\Omega_{Q}\right)$ (so $C$ contains at least one central unit of $\left.\Lambda_{Q}\right)$.

We call any common ideal $C$ of $\Lambda$ and $\Omega$ satisfying (i) a conductor ideal for $\Lambda$ and $\Omega$. We almost always choose $C$ to take advantage of properties (ii) and (iii).

Given an $R$-order $\Omega\left(\Lambda \subseteq \Omega \subseteq \Lambda_{Q}\right)$ and a conductor ideal $C \subseteq J(\Lambda) \cap J(\Omega)$ for $\Lambda$ and $\Omega$, we define $\bar{\Lambda}$ and $\bar{\Omega}$ as in the bottom row of the following conductor square, in which the vertical maps denote surjective ring homomorphisms with kernel $C$, and the horizontal maps denote inclusion. We often refer to $\Lambda$ as the pullback of this conductor square, meaning that the square is commutative and, for $\omega \in \Omega$, if its image $\bar{\omega}$ in $\bar{\Omega}$ satisfies $\bar{\omega} \in \bar{\Lambda}$, then $\omega \in \Lambda$.

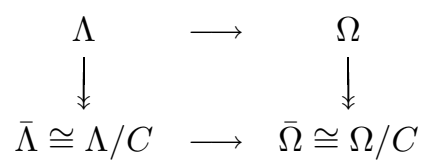

Splitting orders $\Omega$. From now on $\Omega$ always denotes a type of $R$-order $(\Omega \subseteq \Gamma)$ called a "splitting order". The precise definition of a splitting order is given in [LO, Section 5]. We review several of its most important properties, and collect some related notation here for easy reference. (Note. If $\Lambda$ is contained in a maximal $R$ order in $\Lambda_{Q}$ we can always take $\Omega=\Gamma$.) $\Omega$ has a 2 -sided decomposition, compatible with that of $\Gamma$ :

$$
\Gamma=\bigoplus_{k} \Gamma_{k} \quad \text { and } \quad \Omega=\bigoplus_{k} \Omega_{k} \quad\left[\text { each } \Omega_{k} \subseteq \Gamma_{k} \text { and }\left(\Omega_{k}\right)_{Q}=\left(\Gamma_{k}\right)_{Q}\right]
$$

Recall that, since $R$ is semilocal, each $\Gamma_{k}$ is a full $n_{k} \times n_{k}$ matrix ring over a principal left and right ideal domain. Each $\Omega_{k}$ is a full $n_{k} \times n_{k}$ matrix ring over an integral domain [LO, (5.1.2)], but that domain need not be a principal ideal domain. However, $\Omega$ satisfies KS for projective modules [LO, 5.5].

Given any $\Omega$, we let

$$
M_{k i}=M_{k i}^{\prime} \cap \Omega_{k}
$$

Since the maximal ideals of $\Gamma$ and $\Omega$ are in one-to-one correspondence via contraction [LO, Lemma 5.3], $\left\{M_{k i}\right\}$ is the set of all maximal ideals of $\Omega_{k}$.

For each $k$ there is (up to isomorphism) a unique indecomposable projective (left) $\Omega_{k}$ module that we call $B_{k}[\mathrm{LO}, 5.6]$. As with maximal orders, the $\Omega_{Q}=\Lambda_{Q^{-}}$ modules $\left(B_{k}\right)_{Q}$ form a set of representatives for the isomorphism classes of simple $\Lambda_{Q}$-modules. See [LO, 5.5] for more about this.

Part of the definition of "splitting order" is that each natural map $\Omega_{k} / M_{k i} \rightarrow$ $\Gamma_{k} / M_{k i}^{\prime}$ is a bijection. Therefore the simple $\Omega_{k}$-modules coincide with the simple $\Gamma_{k^{-}}$ modules $S_{k i}$. It follows that $s_{k i}$ is the splitting number of $M_{k i}$, that is, the following analog of (3.5.1) holds, where $e_{k}$ denotes an arbitrary primitive idempotent element of $\Omega_{k}$ :

$$
B_{k} / M_{k i} B_{k} \cong \Omega_{k} e_{k} / M_{k i} e_{k} \cong\left(S_{k i}\right)^{s_{k i}}
$$


The decomposition of $\Omega$ in (3.5.5) yields a decomposition $\bar{\Omega}=\bigoplus_{k} \bar{\Omega}_{k}$ where $\bar{\Omega}_{k} \cong \Omega_{k} / C \Omega_{k}$ denotes the image of $\Omega_{k}$ in $\bar{\Omega}$. We set $\bar{B}_{k}=B_{k} / C B_{k}$. This is a projective $\bar{\Omega}_{k}$-module, but need not be indecomposable. There is a unique indecomposable projective $\bar{\Omega}_{k}$-module $\bar{B}_{k i}$ that maps onto $S_{k i}$, and every indecomposable projective $\bar{\Omega}_{k}$-module is isomorphic to some $\bar{B}_{k i}[\mathrm{LO}, 6.19]$. The precise relationship among all these projective modules is given by

$$
\Omega_{k} \cong\left(B_{k}\right)^{n_{k}}, \quad \bar{B}_{k} \cong \bigoplus_{i}\left(\bar{B}_{k i}\right)^{s_{k i}},
$$

where $n_{k}$ is the matrix size of $\Gamma_{k}$ which, as mentioned above, equals the matrix size of $\Omega_{k}$. The second isomorphism follows from (3.5.7) and the fact, for artinian rings, idempotents lift modulo the radical. See [LO, 6.19]. Note that the multiplicities $n_{k}$ and $s_{k i}$ in (3.5.8) are the same for all splitting orders $\Omega$.

The notation and basic facts concerning the two-sided decomposition of each $\bar{\Omega}_{k}$ are given $[\mathrm{LO},(6.18 .1)]$ by:

(3.5.9) $\bar{\Omega}_{k}=\bigoplus_{i} \bar{\Omega}_{k i}$ where each $\bar{\Omega}_{k i}$ is a matrix-local ring whose maximal ideal is $\bar{M}_{k i}$, the image of $M_{k i}$ in $\bar{\Omega}_{k i}$, and whose unique indecomposable projective (left) module we call $\bar{B}_{k i}$

We define a module $[X \subseteq Y]$ over the artinian pair $(\bar{\Lambda}, \bar{\Omega})$ to be a projective $\bar{\Omega}$-module $Y$, together with a $\bar{\Lambda}$-submodule $X$ such that $\bar{\Omega} X=Y$. We therefore have $Y=\bigoplus_{k i}\left(\bar{B}_{k i}\right)^{\rho_{k i}}$ for suitable multiplicities $\rho_{k i}$. We call the array

$$
\rho[X \subseteq Y]=\left(\left(\rho_{k i}\right)\right)=\left(\left(\rho_{k i}[X \subseteq Y]\right)\right)
$$

the array of ranks of $[X \subseteq Y]$. Note that this array is not a matrix because its rows need not all have the same length. Note also that $\rho[X \subseteq Y]$ is completely determined by $Y$. The reason for studying $(\bar{\Lambda}, \bar{\Omega})$-modules is the following slight generalization of a well-known idea.

Let $L$ be a torsionfree $\Lambda$-module. Then we can find a splitting order $\Omega$ such that $\Omega L$ is $\Omega$-projective, that is, projective as an $\Omega$-module [LO, 5.2]. Let $C \subseteq$ $J(\Lambda) \cap J(\Omega)$ be a conductor ideal for $\Lambda$ and $\Omega$. Then we can form the $(\bar{\Lambda}, \bar{\Omega})-$ module

$$
\Phi(L)=[L / C L \subseteq \Omega L / C L]
$$

Now fix a particular splitting order $\Omega(\Lambda \subseteq \Omega \subseteq \Gamma)$, and let $L$ range over all torsionfree $\Lambda$-modules such that $\Omega L$ is $\Omega$-projective. By the package deal theorem for artinian pairs [LO, 6.21], the isomorphism class of $\Phi(L)$ determines $L$ up to $\Lambda$ isomorphism; and the answer to the question of whether a $(\bar{\Lambda}, \bar{\Omega})$-module $[X \subseteq Y]$ is isomorphic to $\Phi(L)$ for some $L$ is determined by the array of ranks $\rho[X \subseteq Y]$. Thus the function $\Phi$ is faithful — and is obviously additive — in the sense of (1.4.1). The class $\mathcal{K}$ of all $(\bar{\Lambda}, \bar{\Omega})$-modules satisfies KS [LO, 6.22]. Therefore we can use $\Phi$ as a function with which to play the package deal game, even when no maximal order in $\Lambda_{Q}$ contains $\Lambda$.

Caution. Many of the results in the companion paper [LO] are developed in more generality than necessary for the present paper, and this results in some slight differences between the standard notation used there and that used here. In particular, $\Lambda$ and $R$ can have nilpotent ideals in some parts of [LO]. Also, the convention in the present paper, that all modules be finitely generated (f.g.) unless otherwise specified, is abandoned in [LO]. 
Readers interested only in the situation that $\Lambda$ is contained in a maximal order may always assume that $\Omega=\Gamma$.

\section{Package Deal Game: Combinatorics}

In this section we show that the TFKS property places very strong combinatorial restrictions on the structure of packages.

The notation $K \mid P$ denotes that $K$ is isomorphic to a direct summand of $P$. The ring $R$ remains semilocal.

Let $\mathcal{S} \subseteq \mathcal{K}$ be classes of modules (over some ring). Suppose that $\mathcal{S}$ and $\mathcal{K}$ are closed under (finite) direct sums, modules in $\mathcal{K}$ can be written as finite direct sums of indecomposables in $\mathcal{K}$, and $\mathrm{KS}$ holds in $\mathcal{K}$. In this discussion we call elements of the subclass $\mathcal{S}$ packages. Since KS holds in $\mathcal{K}$, every package is uniquely expressible (up to isomorphism) as a direct sum of modules in $\mathcal{K}$ that are indecomposable in $\mathcal{K}$. We define an indecomposable package to be a nonzero package that is not isomorphic to a direct sum of two nonzero packages. When does the KS property descend from $\mathcal{K}$ to $\mathcal{S}$ ?

Proposition 4.1. (Keep the preceding terminology.) Suppose that $\mathcal{S}$ is closed under complementation in $\mathcal{K}$; that is

$$
\left.P \cong P^{\prime} \oplus K \quad \text { (with } P, P^{\prime} \in \mathcal{S}, K \in \mathcal{K}\right) \Rightarrow K \in \mathcal{S} .
$$

Then $K S$ holds in $\mathcal{S}$ if and only if, for each indecomposable package $P \in \mathcal{S}$, there is a module $K \in \mathcal{K}$ that is indecomposable in $\mathcal{K}$ and such that $K \mid P$ but $K$ is not isomorphic to a direct summand of any indecomposable package $¥ P$.

We call any such $K$ an indicator summand for $P$.

Proof. (Only if.) Suppose some indecomposable $P$ has no indicator summand, and write $P \cong \bigoplus_{i} K_{i}$ with each $K_{i} \in \mathcal{K}$ indecomposable. Then each $K_{i} \mid P_{i}$ for some indecomposable package $P_{i} \not P$. Then $P \mid \bigoplus_{i} P_{i}$ in $\mathcal{K}$, hence in $\mathcal{S}$ by (4.1.1). This shows that KS fails in $\mathcal{S}$.

(If.) Let $P=\bigoplus_{i} P_{i}^{a_{i}}$ with the $P_{i}$ indecomposable packages in distinct isomorphism classes. Write each $P_{i}$ as a direct sum of indecomposable modules in $\mathcal{K}$. Then $\mathrm{KS}$ in $\mathcal{K}$, together with the existence of indicator summands, yields uniqueness of the $a_{i}$. Hence KS holds for packages.

Definition 4.2. We state our next KS criterion in terms of numerical invariants we call "rank ratios". Fix a splitting order $\Omega(\Lambda \subseteq \Omega \subseteq \Gamma)$ and a conductor ideal $C$ for $\Lambda$ and $\Omega$, thus defining $\bar{\Lambda}$ and $\bar{\Omega}$ as in Notation 3.5. Recall that the maximal ideals of each $\Gamma_{k}$ are denoted by $M_{k i}^{\prime}$, and the splitting number $s_{k i}$ of $M_{k i}^{\prime}$ is the integer defined in (3.5.1). Each $(\bar{\Lambda}, \bar{\Omega})$-module $[X \subseteq Y]$ has an associated array of ranks $\left(\left(\rho_{k i}\right)\right)$, as defined in (3.5.10). We define the rank ratios associated with a $(\bar{\Lambda}, \bar{\Omega})$-module $[X \subset Y]$ whose inclusion is proper to be the following rational numbers:

$$
\rho_{k i} / s_{k i}=\rho_{k i}[X \subset Y] / s_{k i}
$$

Lemma 4.3 (Fundamental Combinatorial Lemma). (Keep the notation in Definition 4.2.) Let $\mathcal{S}$ be a collection of torsionfree (left) $\Lambda$-modules that is closed under direct sums and direct summands, such that $\Omega L$ is $\Omega$-projective for every $L \in \mathcal{S}$, and such that $\Omega \in \mathcal{S}$. Then $K S$ holds in $\mathcal{S}$ if and only if, for each $k$, the rings $\bar{\Omega}_{k i}$ (hence their indecomposable projective modules $\bar{B}_{k i}$ ) can be renumbered in such a 
way that, for all indecomposable $(\bar{\Lambda}, \bar{\Omega})$-modules $[X \subset Y]$ with proper inclusion, the associated rank ratios (4.2.1) satisfy the following two conditions.

(i) $\rho_{k 1} / s_{k 1}=\max \left\{\rho_{k 1} / s_{k 1}, \rho_{k 2} / s_{k 2}, \ldots\right\}$; and

(ii) $\rho_{k 1} / s_{k 1}$ is an integer.

We call $(k, 1)$ the $k$-indicator pair. Note that the definition does not require the renumbering to be unique. However, once the renumbering is done, conditions (i) and (ii) must hold for all indecomposable $[X \subset Y]$ whose inclusion is proper.

Proof. We prove the lemma by invoking Proposition 4.1. Let $\Phi$ be the function defined in (3.5.11), and let $\mathcal{K}$ be the set of all $(\bar{\Lambda}, \bar{\Omega})$-modules that are direct summands of $(\bar{\Lambda}, \bar{\Omega})$-modules in $\Phi(\mathcal{S})$. Since KS holds for $(\bar{\Lambda}, \bar{\Omega})$-modules [LO, 6.22], it holds in $\mathcal{K}$.

The map $\Phi: \mathcal{S} \rightarrow \mathcal{K}$ is an additive, faithful imbedding, as explained after (3.5.11); and we therefore identify $\mathcal{S}$ with $\Phi(\mathcal{S})$ for the rest of this proof. In order to apply Proposition 4.1 we need to verify the complementation condition (4.1.1). [One additional technicality needs to be noted. In Proposition $4.1, \mathcal{K}$ is a family of actual modules over a ring. In fact, as noted in the proof of [LO, 6.22], $(\bar{\Lambda}, \bar{\Omega})$ modules can be viewed as actual modules over an artinian lower triangular matrix ring. Having made this observation, we shall have no further occasion to use this way of viewing $(\bar{\Lambda}, \bar{\Omega})$-modules.]

The package deal theorem for $(\bar{\Lambda}, \bar{\Omega})$-modules $[\mathrm{LO}, 6.21]$ states that a $(\bar{\Lambda}, \bar{\Omega})$ module $[X \subseteq Y]$ satisfies $[X \subseteq Y] \cong \Phi(L)$ for some torsionfree $\Lambda$-module $L \in \mathcal{S}$ if and only if:

$$
\left(\rho_{k 1}, \rho_{k 2}, \ldots\right) \in \mathbb{N} \cdot\left(s_{k 1}, s_{k 2}, \ldots\right) \quad(\forall k) .
$$

In other words, every rank ratio $\rho_{k i} / s_{k i}$ is an integer depending only on $k$. Complementation condition (4.1.1) follows immediately from (4.3.1). We call $(\bar{\Lambda}, \bar{\Omega})$ modules in $\mathcal{S}$ packages.

For a $(\bar{\Lambda}, \bar{\Omega})$-module $[X \subseteq Y]$ let $r_{k}[X \subseteq Y]$ be the smallest integer greater than or equal to $\max \left\{\rho_{k 1} / s_{k 1}, \rho_{k 2} / s_{k 2}, \ldots\right\}$, and then define the package $P[X \subseteq Y]$ to be

$$
P[X \subseteq Y]=[X \subseteq Y] \oplus\left(\bigoplus_{k i}\left[\bar{B}_{k i} \subseteq \bar{B}_{k i}\right]^{a_{k i}}\right)
$$

where $a_{k i} / s_{k i}=r_{k}[X \subseteq Y]-\rho_{k i}[X \subseteq Y] / s_{k i}$. This is indeed a package, by (4.3.1). Note that $a_{k i}=0$ if and only if

$$
\rho_{k i} / s_{k i}=\max \left\{\rho_{k 1} / s_{k 1}, \rho_{k 2} / s_{k 2}, \ldots\right\}=r_{k}[X \subseteq Y] \in \mathbb{N} .
$$

Note also that

(4.3.4) If $[X \subseteq Y]$ is an indecomposable $(\bar{\Lambda}, \bar{\Omega})$-module, then $P[X \subseteq Y]$ is an indecomposable package.

This is true since KS holds in mod- $(\bar{\Lambda}, \bar{\Omega})$, and since (by (4.3.1)), the integers $a_{k i}$ give $P[X \subseteq Y]$ the smallest possible ranks allowable for any package containing $[X \subseteq Y]$ as a direct summand.

Now assume that KS holds in $\mathcal{S}$. Let $\bar{B}_{k}$ and $\bar{B}_{k i}$ be as in (3.5.8). By Proposition 4.1 each of the indecomposable packages $\left[\bar{B}_{k} \subseteq \bar{B}_{k}\right]$ has an indicator summand, say $\left[\bar{B}_{k 1} \subseteq \bar{B}_{k 1}\right]$. We claim that conditions (i) and (ii) hold. Let $[X \subset Y]$ be an indecomposable $(\bar{\Lambda}, \bar{\Omega})$-module with proper inclusion, and consider $P[X \subset Y]$, as 
displayed in (4.3.2). Since $\left[\bar{B}_{k 1} \subseteq \bar{B}_{k 1}\right]$ is an indicator summand for $\left[\bar{B}_{k} \subseteq \bar{B}_{k}\right]$, the former cannot be isomorphic to a direct summand of the indecomposable package $P[X \subset Y]$. Therefore $a_{k 1}=0$. Therefore the desired conditions are furnished by (4.3.3).

Conversely, suppose that renumbering has been done is such a way that (i) and (ii) hold. Since each $\left[\bar{B}_{k} \subseteq \bar{B}_{k}\right]=P\left[\bar{B}_{k 1} \subseteq \bar{B}_{k 1}\right]$, it suffices to show that every other indecomposable package has the form $P[X \subset Y]$ for some indecomposable $[X \subset Y]$ with $a_{k 1}=0$. For then, in the terminology of Proposition 4.1, every indecomposable package has an indicator summand, namely $\left[\bar{B}_{k 1} \subseteq \bar{B}_{k 1}\right]$ for $\left[\bar{B}_{k} \subseteq \bar{B}_{k}\right]$, and $[X \subset Y]$ for $P[X \subset Y]$.

So let $P^{\prime}=\left[X^{\prime} \subseteq Y^{\prime}\right]$ be any indecomposable package not of the form $\left[\bar{B}_{k} \subseteq \bar{B}_{k}\right]$. Then $X^{\prime}$ is not an $\bar{\Omega}$-module, and therefore the inclusion $\left[X^{\prime} \subseteq Y^{\prime}\right]$ is proper. It follows that, when $P^{\prime}$ is written as a direct sum of indecomposable $(\bar{\Lambda}, \bar{\Omega})$-modules, some term must have the form $[X \subset Y]$. We claim that $P^{\prime}=P[X \subset Y]$. We have

$$
P^{\prime}=[X \subset Y] \oplus\left(\bigoplus_{j}\left[U_{j} \subset V_{j}\right]\right) \oplus\left(\bigoplus_{k i}\left[\bar{B}_{k i} \subseteq \bar{B}_{k i}\right]^{d_{k i}}\right)
$$

for suitable indecomposable $(\bar{\Lambda}, \bar{\Omega})$-modules $\left[U_{j} \subset V_{j}\right]$ and nonnegative integers $d_{k i}$. To complete the proof of the claim it suffices, by indecomposability of $P^{\prime}$, to prove that $P[X \subset Y] \mid P^{\prime}$. This, in turn, is equivalent to proving that $a_{k i} \leq d_{k i}$ for every $k$ and $i$, as one can see by comparing (4.3.5) with (4.3.2).

As usual, let $\rho_{k i}$ denote the $(k, i)$ entry of the array of ranks of $[X \subset Y]$, and similarly let $\tau_{k i j}$ denote the $(k, i)$ entry of the array of ranks of $\left[U_{j} \subset V_{j}\right]$. Since $P^{\prime}$ is a package its $(k, 1)$ rank ratio equals its $(k, i)$ rank ratio for every $(k, i)$. In other words,

$$
\frac{\rho_{k 1}}{s_{k 1}}+\left(\sum_{j} \frac{\tau_{k 1 j}}{s_{k 1}}\right)+\frac{d_{k 1}}{s_{k 1}}=\frac{\rho_{k i}}{s_{k i}}+\left(\sum_{j} \frac{\tau_{k i j}}{s_{k i}}\right)+\frac{d_{k i}}{s_{k i}} .
$$

Now consider $P[X \subset Y]$. Since we are assuming that conditions (i) and (ii) hold, we have $a_{k 1}=0$ as observed above (4.3.3). Therefore equating the $(k, 1)$ and $(k, i)$ rank ratios of the package $P[X \subset Y]$ yields $\rho_{k 1} / s_{k 1}=\rho_{k i} / s_{k i}+a_{k i} / s_{k i}$. Substituting this value of $\rho_{k 1} / s_{k 1}$ into (4.3.6) enables us to cancel $\rho_{k i} / s_{k i}$ from both sides. Condition (i) implies that each $\tau_{k 1 j} / s_{k 1} \geq \tau_{k i j} / s_{k i}$. Therefore, after replacing the equality in (4.3.6) by $\leq$, we can delete the terms $\tau_{k 1 j} / s_{k 1}$ and $\tau_{k i j} / s_{k i}$. We can also delete the term $d_{k 1} / s_{k 1}$ from the left-hand side. The inequality that results is $a_{k i} \leq d_{k i}$, as desired.

When proving the previous claim we noted that each $a_{k 1}=0$; so the proof of the lemma is now complete.

Remark 4.4. If $\Gamma$ is module-finite over $R$, we can take $\mathcal{S}$ to be the collection of all torsionfree $\Lambda$-modules, and $\Omega=\Gamma$.

\section{Only One Singular Maximal Ideal}

In this section we reduce the KSM and TFKS problems to the case that $Z(\Lambda)$ is a local ring. $R$ is always assumed semilocal. If $\mathcal{M}$ is a set of maximal ideals of $R$, then $R_{\mathcal{M}}, \Lambda_{\mathcal{M}}, \ldots$ denotes the localization that inverts the multiplicatively closed set $R-\bigcup \mathcal{M}$. 
Lemma 5.1. (i) "Centers localize", that is, $Z\left(\Lambda_{S}\right)=Z(\Lambda)_{S}$ for every multiplicatively closed subset $S$ of $R$.

(ii) $\Lambda$ is an indecomposable ring if and only if $Z(\Lambda)$ is.

The proof of statement (i) is a routine exercise whose result we shall use often. Statement (ii) holds because indecomposability of a ring can be stated in terms of central idempotents.

Proposition 5.2. Let $\mathcal{M}$ be a set of maximal ideals of $R, X$ a $\Lambda_{\mathcal{M}}$-module, and $\Omega$ a splitting order $(\Lambda \subseteq \Omega \subseteq \Gamma)$. Then there exists a $\Lambda$-module $M$, unique up to isomorphism, such that

(i) $M_{\mathcal{M}} \cong X$;

(ii) For all maximal ideals $\mathbf{n} \notin \mathcal{M}, M_{\mathbf{n}}$ is a projective $\Omega_{\mathbf{n}}$-module (and therefore the natural map $M_{\mathbf{n}} \rightarrow\left(M_{\mathbf{n}}\right)_{Q}$ is one-to-one); and

(iii) $M_{Q} \cong X_{Q}$.

Moreover, $M$ is an indecomposable $\Lambda$-module if and only if $X$ is an indecomposable $\Lambda_{\mathcal{M}}$-module.

We call $M$ the $\Lambda$-module canonically determined by $X$ (and $\Omega$ ).

Proof. For the first approximation to the desired module $M$, choose the $\Lambda$-module generated by some finite set of $\Lambda_{\mathcal{M}}$-generators of $X$. Then (i) and (iii) hold [see the proof of (3.2.3)].

In order to satisfy (ii), fix $\mathbf{n} \notin \mathcal{M}$. Since $R$ is semilocal, it suffices to show that $M$ can be changed so that $M_{\mathbf{n}}$ is replaced by a projective $\Lambda_{\mathbf{n}}$-module $Y(\mathbf{n})$ without changing any other $M_{\mathbf{m}}$. By the package deal theorem for localizations of modules [LO, 2.9] we can replace $M_{\mathbf{n}}$ by any $\Lambda_{\mathbf{n}}$-module $Y(\mathbf{n})$ such that $Y(\mathbf{n})_{Q} \cong\left(M_{\mathbf{n}}\right)_{Q}$. Now an easy check shows $\Omega_{\mathbf{n}}$ is a splitting $R_{\mathbf{n}}$-order and, of course, $\left(\Lambda_{\mathbf{n}}\right)_{Q}=\left(\Omega_{\mathbf{n}}\right)_{Q}$. So there is a projective $\Omega_{\mathbf{n}}$ module $Y(\mathbf{n})$ (unique up to isomorphism) such that $Y(\mathbf{n})_{Q} \cong\left(M_{\mathbf{n}}\right)_{Q}[\mathrm{LO}, 5.5]$. Thus the desired $M$ exists.

To prove uniqueness of $M$ (up to isomorphism) it suffices, since $R$ is semilocal, to prove uniqueness of every $M_{\mathbf{m}}$. For $\mathbf{m} \in \mathcal{M}$ this is clear; and for other $\mathbf{m}$ it holds by uniqueness of $Y(\mathbf{m})$.

Now let $X$ be indecomposable, and suppose that $M \cong N \oplus N^{\prime}$. By indecomposability of $X \cong M_{\mathcal{M}}$ we have (say) $N_{\mathcal{M}}=0$. We claim that $N=0$. It suffices to show that $N$ is the (unique) $\Lambda$-module canonically determined by 0 . We already have property (i) (with $X=0$ ), and (ii) holds since direct summands of projective modules are again projective. Thus it remains to prove that $N_{Q}=0$. It suffices to prove that $N_{\mathbf{p}}=0$ for every minimal prime $\mathbf{p}$ [LO, 2.3(i)]. Case 1: $\mathbf{p}$ is contained in some $\mathbf{m} \in \mathcal{M}$. Then $N_{\mathbf{p}}=0$ since $N_{\mathcal{M}}=0$. Case 2: $\mathbf{p}$ is contained in no $\mathbf{m} \in \mathcal{M}$. Then $N_{\mathbf{p}} \subseteq M_{\mathbf{p}} \subseteq X_{\mathbf{p}}$, and $X_{\mathbf{p}}=0$ since $R_{\mathbf{m}} \otimes_{R} R_{\mathbf{p}}=0$ when $\mathbf{m}$ does not contain $\mathbf{p}[\mathrm{LO}, 2.3]$.

Conversely, suppose that $M$ is indecomposable, and write $X \cong Y \oplus Y^{\prime}$. Letting $N, N^{\prime}$ be the $\Lambda$-modules canonically determined by $Y, Y^{\prime}$, we obtain that $N \oplus N^{\prime}$ is the $\Lambda$-module canonically determined by $Y \oplus Y^{\prime}$, and therefore $M \cong N \oplus N^{\prime}$. Indecomposability of $M$ yields (say) $N=0$, hence $Y=N_{\mathcal{M}}=0$.

Corollary 5.3. Suppose that $K S$ holds in the class $\mathcal{S}(\Omega)$ of torsionfree $\Lambda$-modules $M$ such that $\Omega M$ is $\Omega$-projective. Then, for every set $\mathcal{M}$ of maximal ideals of $R, K S$ holds in the class $\mathcal{S}\left(\Omega_{\mathcal{M}}\right)$ of torsionfree $\Omega_{\mathcal{M}}$-modules $N$ such that $\Omega_{\mathcal{M}} N$ is $\Omega_{\mathcal{M} \text {-projective. }}$ 
Proof. Let $X \cong \bigoplus_{i} X_{i} \cong \bigoplus_{j} Y_{j}$ be decompositions of a $\Lambda_{\mathcal{M}}$-module in $\mathcal{S}\left(\Omega_{\mathcal{M}}\right)$, with every $X_{i}, Y_{j}$ indecomposable. Let $M, M_{i}, N_{j}$ respectively be the $\Lambda$-modules canonically determined by $X, X_{i}, Y_{j}$, as defined in Proposition 5.2. By that proposition, every $M_{i}, N_{j}$ is indecomposable. Since conditions (i)-(iii) of the proposition are preserved by direct sums, the uniqueness part of the proposition shows that $M \cong \bigoplus_{i} M_{i} \cong \bigoplus_{j} N_{j}$. We can now apply KS in $\mathcal{S}(\Omega)$ to deduce KS in $\mathcal{S}\left(\Omega_{\mathcal{M}}\right)$.

The next lemma is the crux of our reduction to the local case.

Lemma 5.4. Suppose that $\Lambda$ is an indecomposable ring, and $R$ has at least two maximal ideals that are singular with respect to $\Lambda$. Then TFKS fails for $\Lambda$.

Proof. Let $\mathbf{m} \neq \mathbf{n}$ be maximal ideals of $R$ that are singular with respect to $\Lambda$. Then $\mathbf{m}$ and $\mathbf{n}$ contain the kernel of the natural map $r \rightarrow r \cdot 1_{\Lambda}$ of $R \rightarrow \Lambda$, and therefore the natural images of $\mathbf{m}$ and $\mathbf{n}$ in $\Lambda$ are distinct maximal ideals of $R \cdot 1_{\Lambda}$ that are singular with respect to $\Lambda$. Since $Z(\Lambda)$ is integral over $R \cdot 1_{\Lambda}$, we see that $Z(\Lambda)$ has at least two maximal ideals that are singular with respect to $\Lambda$.

We can choose a splitting order $\Omega(\Lambda \subset \Omega \subseteq \Gamma)$ that is large enough so that the inclusions $\Omega_{\mathbf{m}} \supset \Lambda_{\mathbf{m}}$ and $\Omega_{\mathbf{n}} \supset \Lambda_{\mathbf{n}}$ are proper [LO, 5.2].

We first prove the lemma under the additional assumption that $\Lambda$ is a prime ring; that is, $\Gamma=\Gamma_{1}$ in the decomposition (2.1.2). This is stronger than assuming that $\Lambda$ is an indecomposable ring. We may also assume that $R=Z(\Lambda)$, because of the comments in the first paragraph of this proof.

Every $R$-module of finite length is the direct sum of its nonzero localizations at maximal ideals of $R$. Hence, in terms of our Semilocal Notation 3.5, the artinian pair $[\bar{\Lambda} \subset \bar{\Omega}]$ has a decomposition

$$
[\bar{\Lambda} \subset \bar{\Omega}]=\left[\bar{\Lambda}_{\mathbf{m}} \subset \bar{\Omega}_{\mathbf{m}}\right] \oplus\left[\bar{\Lambda}_{\mathbf{n}} \subset \bar{\Omega}_{\mathbf{n}}\right] \oplus \cdots
$$

and therefore:

(5.4.2) There are indecomposable direct summands $[X \subset Y]$ and $\left[X^{\prime} \subset Y^{\prime}\right]$, of $\left[\bar{\Lambda}_{\mathbf{m}} \subset \bar{\Omega}_{\mathbf{m}}\right]$ and $\left[\bar{\Lambda}_{\mathbf{n}} \subset \bar{\Omega}_{\mathbf{n}}\right]$ respectively, whose inclusions are strict.

Now consider the arrays of ranks $\rho[X \subset Y]$ and $\rho\left[X^{\prime} \subset Y^{\prime}\right]$, as defined in (3.5.10). Since $\Gamma=\Gamma_{1}$, hence $\Omega=\Omega_{1}$ in the decomposition (3.5.5), each of these arrays consists of a single row. Moreover every indecomposable projective $\bar{\Omega}_{1}$-module $\bar{B}_{1 i}$ is isomorphic to a direct summand of a unique one of the terms $\bar{\Omega}_{\mathbf{m}}, \bar{\Omega}_{\mathbf{n}}, \ldots$ on the right-hand side of (5.4.1).

It follows that the coordinates containing the nonzero entries of $\rho[X \subset Y]$ are disjoint from those containing the nonzero entries of $\rho\left[X^{\prime} \subset Y^{\prime}\right]$. Therefore the maximum values of the rank ratios $\rho_{1 i}[X \subset Y] / s_{1 i}$ and $\rho_{1 i}\left[X^{\prime} \subset Y^{\prime}\right] / s_{1 i}$ occur for distinct subscripts $i$. It follows from our Fundamental Combinatorial Lemma 4.3(i) that TFKS fails for $\Lambda$.

Now we consider the general case of the lemma: The ring $\Lambda$ is indecomposable but not necessarily prime. We claim that the two singular maximal ideals $\mathbf{m}, \mathbf{n}$ can be chosen to contain a common minimal prime ideal $\mathbf{p}$ of $R$. Again we may assume that $R=Z(\Lambda)$.

Let $\mathbf{m} \neq \mathbf{n}$ be any maximal ideals of $R$ such that $\Lambda_{\mathbf{m}} \neq \Gamma_{\mathbf{m}}$ and $\Lambda_{\mathbf{n}} \neq \Gamma_{\mathbf{n}}$. Since $\Lambda$ is an indecomposable ring, so is its center $R$ [Lemma 5.1], so spec $(R)$ is connected. Hence there is a sequence $\mathbf{m}=\mathbf{m}(0), \mathbf{m}(1), \ldots, \mathbf{m}(t)=\mathbf{n}$ of maximal ideals such that, for each $i$, the maximal ideals $\mathbf{m}(i-1), \mathbf{m}(i)$ contain a common minimal 
prime $\mathbf{p}(i)$. Moreover we can suppose (after perhaps shortening the sequence and renaming $\mathbf{n}$ ) that $\Lambda_{\mathbf{m}(i)}=\Gamma_{\mathbf{m}(i)}$ except for $i=0$ and $i=t$. The claim will be proved if we show that $\mathbf{p}(1)=\ldots=\mathbf{p}(t)$.

For $i \neq 0$ and $i \neq t$ we have $\Lambda_{\mathbf{m}(i)}=\Gamma_{\mathbf{m}(i)}$. Moreover, since centers localize, $R_{\mathbf{m}(i)}=Z(\Lambda)_{\mathbf{m}(i)}=Z\left(\Lambda_{\mathbf{m}(i)}\right)=Z\left(\Gamma_{\mathbf{m}(i)}\right)$. Since $\Gamma$ is a direct sum of prime rings, $Z(\Gamma)$ is a direct sum of integral domains, so each maximal ideal of $Z(\Gamma)$ contains a unique minimal prime. Hence the same is true of $Z(\Gamma)_{\mathbf{m}(i)}=R_{\mathbf{m}(i)}$. This shows that $\mathbf{p}(i)_{\mathbf{m}(i)}=\mathbf{p}(i+1)_{\mathbf{m}(i)}$ and hence $\mathbf{p}(i)=\mathbf{p}(i+1)$, completing the proof of the claim.

We may now assume that $\mathbf{m}$ and $\mathbf{n}$ contain a common minimal prime $\mathbf{p}$ of $R$. Beginning with the decomposition $\Omega=\bigoplus_{k} \Omega_{k}$, let $\Lambda_{k}$ be the projection of $\Lambda$ in $\Omega_{k}$. Since $R$ has no nilpotent elements, the set of minimal primes of $R$ is the set of kernels $\operatorname{ker}\left(R \rightarrow \Omega_{k}\right)$. Renumber the $\Omega_{k}$ so that $\mathbf{p}=\operatorname{ker}\left(R \rightarrow \Omega_{1}\right)$. Then every torsionfree $\Lambda_{1}$-module is a torsionfree $\Lambda$-module. (For this purpose, the most convenient definition of a torsionfree $\Lambda$-modules is "any submodule of a free $\Lambda$ module". See Definitions 1.2.) By the prime case of the lemma, $\Lambda_{1}$ fails to satisfy TFKS [even if the natural image of $R$ in $\Lambda_{1}$ is properly smaller than $Z\left(\Lambda_{1}\right)$ ], and hence so does $\Lambda$.

Lemma 5.5. Let $M$ be a $\Lambda$-module and $\mathbf{n}$ a maximal ideal of $R$. Suppose a $\Lambda_{\mathbf{n}^{-}}$ module $F$ of finite $R_{\mathbf{n}}$-length is a $\Lambda_{\mathbf{n}}$-direct summand of $M_{\mathbf{n}}$. Then $F$ is isomorphic to a $\Lambda$-direct summand of $M$ (and has finite length as an $R$-module).

Proof. First we note that $F$ has finite length as an $R$-module. For the unique simple $R_{\mathbf{n}}$-module $R_{\mathbf{n}} / \mathbf{n}_{\mathbf{n}}$ is isomorphic to $R / \mathbf{n}$, hence any $R_{\mathbf{n}^{-}}$-composition of $F$ is also an $R$-composition series.

By hypothesis there exist $\Lambda_{\mathbf{n}}$-homomorphisms $i^{\prime}: F \rightarrow M_{\mathbf{n}}$ and $\pi^{\prime}: M_{\mathbf{n}} \rightarrow F$ such that $\pi^{\prime} i^{\prime}=1_{F}$. Since $F=F_{\mathbf{n}}$ is a (finitely presented) $R$-module we have $\operatorname{Hom}_{R_{\mathbf{n}}}\left(M_{\mathbf{n}}, F\right)=\operatorname{Hom}_{R}(M, F)_{\mathbf{n}}$. So $i^{\prime}=i / d$ for some $i \in \operatorname{Hom}_{R}(M, F)$ and $d \in R-\mathbf{n}$. Let $\pi: M \rightarrow F$ be the composition of the natural map $M \rightarrow M_{\mathbf{n}}$ with $\pi^{\prime}$. Then $\pi i=d \cdot 1_{F}$. Since multiplication by $d$ is an automorphism of $F$, the proof is complete.

Since centers localize and rings with local centers are indecomposable, our next result reduces the KS problems to the case that $Z(\Lambda)$ is a local ring.

Theorem 5.6. Assume that $\Lambda$ is an indecomposable ring and $\Lambda \neq \Gamma$. Then TFKS (respectively KSM) holds for $\Lambda$ if and only if

(i) There is exactly one maximal ideal $\mathbf{n}$ of $Z(\Lambda)$ such that $\Lambda_{\mathbf{n}} \neq \Gamma_{\mathbf{n}}$; and

(ii) TFKS (respectively KSM) holds for $\Lambda_{\mathbf{n}}$.

Proof. Let $R=Z(\Lambda)$. If TFKS holds for $\Lambda$, then condition (i) holds by Lemma 5.4. Condition (ii) holds in the torsionfree (respectively KSM) case by Lemma 3.2 and the fact that "genus" is the same as "isomorphism class" when $R$ is semilocal.

Conversely, suppose that (i) and (ii) hold, and consider two finite direct sums of $\Lambda$-modules

$$
\bigoplus_{i} M_{i} \cong \bigoplus_{j} N_{j} \quad\left(\text { each } M_{i}, N_{j} \text { indecomposable }\right) .
$$

We do the torsionfree case first. Let $\Omega$ be a splitting order $(\Lambda \subseteq \Omega \subseteq \Gamma)$ such that each $\Omega M_{i}$ and $\Omega N_{j}$ is a projective $\Omega$-module [LO, 5.2]. 
In order to apply hypothesis (ii) we need to show that each $\Lambda_{\mathbf{n}}$-module $\left(M_{i}\right)_{\mathbf{n}}$ and $\left(N_{j}\right)_{\mathbf{n}}$ is indecomposable. In fact we show that $M_{i}$ is the $\Lambda$-module canonically determined by $\left(M_{i}\right)_{\mathbf{n}}$, as defined in Proposition 5.2. (The analog for $N_{j}$ holds similarly.) To see that $\left(M_{i}\right)_{\mathbf{m}}$ is $\Lambda_{\mathbf{m}}$-projective whenever $\mathbf{m} \neq \mathbf{n}$, recall that $\Lambda_{\mathbf{m}}=$ $\Omega_{\mathbf{m}}=\Gamma_{\mathbf{m}}$, and every $\Omega M_{i}$ is $\Omega$-projective. Then every $\left(M_{i}\right)_{\mathbf{m}}$ is a projective $\Omega_{\mathbf{m}}\left(=\Lambda_{\mathbf{m}}\right)$-module. Thus it remains to show that $\left(M_{i}\right)_{Q} \cong\left(\left(M_{i}\right)_{\mathbf{n}}\right)_{Q}$. This follows if we can show that $R_{Q}=\left(R_{\mathbf{n}}\right)_{Q}$. Since $\Lambda$ is an indecomposable ring, so is $R=Z(\Lambda)$ (Lemma 5.1). If we can show that $\mathbf{n}$ contains every minimal prime ideal of $R$, we will have $R_{Q}=\left(R_{\mathbf{n}}\right)_{Q}$.

Since $\Omega$ is a splitting order, it is a direct sum of prime rings. Hence $Z(\Omega)$ is a direct sum of integral domains. It follows that every maximal ideal of $Z(\Omega)$ contains a unique minimal prime, hence the same is true of $Z\left(\Omega_{\mathbf{m}}\right)=Z\left(\Lambda_{\mathbf{m}}\right)=R_{\mathbf{m}}$ if $\mathbf{m} \neq \mathbf{n}$. Thus every maximal ideal $\mathbf{m} \neq \mathbf{n}$ of $R$ contains a unique minimal prime. Therefore, if $\mathbf{n}$ failed to contain some minimal prime, $R$ would decompose into a direct sum of two rings, contradicting indecomposability of $R$.

Now that we have proved that each $M_{i}$ is the $\Lambda$-module canonically determined by $\left(M_{i}\right)_{\mathbf{n}}$ (and the analog of this for each $N_{j}$ ), we have that every $\left(M_{i}\right)_{\mathbf{n}}$ and $\left(N_{j}\right)_{\mathbf{n}}$ is indecomposable. Thus, by TFKS for $\Lambda_{\mathbf{n}}$ the number of $M_{i}$ equals the number of $N_{j}$, and we can renumber these direct summands in such a way that each $\left(M_{i}\right)_{\mathbf{n}} \cong\left(N_{i}\right)_{\mathbf{n}}$. Since the $M_{i}$ and $N_{i}$ are the $\Lambda$-modules canonically determined by $\left(M_{i}\right)_{\mathbf{n}}$ and $\left(N_{i}\right)_{\mathbf{n}}$ respectively, it follows that each $M_{i} \cong N_{i}$; that is, TFKS holds for $\Lambda$.

Now we consider KSM. The proof in this case is a more technical version of the previous one. We assume, first, that no $M_{i}$ nor $N_{j}$ has finite length.

Define the torsion submodule of a $\Lambda$-module $M$ to be $t(M)=\operatorname{ker}\left(M \rightarrow M_{Q}\right)$. This is an $R$-module of finite length, since it is annihilated by an element $d$ of $R-\cup Q$, hence is a (finitely generated) module over the artinian ring $R / R d$.

Now consider two direct sums of $\Lambda$-modules, as in (5.6.1). Let $\mathbf{m}$ be a maximal ideal of $R$ other than $\mathbf{n}$. We claim that each $\left(M_{i}\right)_{\mathbf{m}}$ and $\left(N_{j}\right)_{\mathbf{m}}$ is a projective $\Lambda_{\mathbf{m}}$-module.

The normalization $\Gamma$ of $\Lambda$ is a maximal order over its center, the normalization $\tilde{R}$ of $R$ in $Z\left(\Lambda_{Q}\right)$ [LO, (4.1.2)], and therefore $\Gamma_{\mathbf{m}}$ is a maximal order over $\tilde{R}_{\mathbf{m}}$ [GL '88, 2.7], or [LO, 4.3]. Moreover, torsionfree modules over maximal orders are projective [R $75,(21.4)]$. Since $\Lambda_{\mathbf{m}}=\Gamma_{\mathbf{m}}$, each $P_{i}=\left(M_{i} / t\left(M_{i}\right)\right)_{\mathbf{m}}=\left(M_{i}\right)_{\mathbf{m}} / t\left(M_{i}\right)_{\mathbf{m}}$ is a projective $\Lambda_{\mathbf{m}}$-module, and therefore $\left(M_{i}\right)_{\mathbf{m}} \cong P_{i} \oplus t\left(M_{i}\right)_{\mathbf{m}}$. Since $t\left(M_{i}\right)_{\mathbf{m}}$ has finite $R_{\mathrm{m}}$-length, Lemma 5.5 shows that it is a direct summand of $M_{i}$, of finite length. Since $M_{i}$ is indecomposable, and not of finite length, we have $t\left(M_{i}\right)_{\mathbf{m}}=0$, proving the claim for $\left(M_{i}\right)_{\mathbf{m}}$. The proof for $\left(N_{j}\right)_{\mathbf{m}}$ is the same.

Now let $\Omega$ be a splitting order $(\Lambda \subseteq \Omega \subseteq \Gamma)$, such that the torsionfree modules $\Omega \cdot\left(M_{i} / t\left(M_{i}\right)\right)$ and $\Omega \cdot\left(N_{i} / t\left(N_{i}\right)\right)$ are $\Omega$-projective. As in the torsionfree case, each $M_{i}$ and $N_{j}$ is the $\Lambda$-module canonically determined by its $\mathbf{n}$-localization, and we finish the proof as in the torsionfree case.

It remains to consider the possibility that (say) some $M_{i}$ has finite length as an $R$-module. Then it has finite length as a $\Lambda$-module, and therefore has a local endomorphism ring. Most of the standard proofs of the classical KS theorem for modules of finite length show that $M_{i}$ is isomorphic to some $N_{j}$, and the isomorphism in (5.6.1) still holds when this isomorphic pair of summands are cancelled. After a finite number of such cancellations, the proof is reduced to the "no summands of finite length" case previously considered. 


\section{LOCAL KSM}

In this section we finish the proof of our necessary and sufficient conditions for $\Lambda$ to satisfy KSM, by proving it when $Z(\Lambda)$ is a local ring.

Throughout this section $R$ is semilocal. Recall the nontriviality assumption, in Notation 2.1, that $Z(\Lambda)$ has no artinian ring-direct summands. This implies that $\Lambda$ has no $R$-submodules of finite length and no $\Lambda$-submodules of finite length [GL '89, (1.4.3)].

Notation 6.1 (completions). This section makes extensive use of $J(R)$-adic completions, where $J(R)$ denotes the Jacobson radical of $R$. We denote $J(R)$-adic completion of an $R$-module $M$ by $\hat{M}$ or, of a more complicated expression, by $(\ldots)^{\wedge}$. For an $\hat{R}$-module $X, X_{Q}$ denotes the localization of $X$ with respect to $R-\bigcup Q(R)$ (rather than the analog of this with respect to $\hat{R}$ ).

We remind the reader that some extra care is necessary in the situation that $\Lambda$ is not contained in a maximal $R$-order in $\Lambda_{Q}$, because $\hat{\Lambda}$ can then have nilpotent ideals. The necessary modifications of standard results are worked out in detail in $[\mathrm{LO}, \S \S 3,6]$. We now give a brief review of the needed results and notation.

The $J(R)$-adic completion of $\Lambda$ can be canonically identified with the $J(Z(\Lambda))$ adic completion of $\Lambda$ [LO, 6.2]. This is what we usually mean, in this section, when we say we may assume that $R=Z(\Lambda)$.

Let $\Omega$ be any splitting $R$-order $(\Lambda \subseteq \Omega \subseteq \Gamma)$, and recall the compatible two-sided decompositions $\Gamma=\bigoplus_{k} \Gamma_{k}$ and $\Omega=\bigoplus_{k} \Omega_{k}$ in (3.5.5). We denote the maximal ideals of $\Gamma_{k}$ by $M_{k i}^{\prime}$. The contraction relations

$$
M_{k i}^{\prime} \rightarrow M_{k i}=M_{k i}^{\prime} \cap \Omega_{k} \rightarrow \mathbf{m}_{k i}=M_{k i} \cap Z\left(\Omega_{k}\right)
$$

establish bijections between the sets of maximal ideals of $\Gamma_{k}, \Omega_{k}$ and $Z\left(\Omega_{k}\right)$. We denote the $\mathbf{m}_{k i}$-adic completion of a $Z\left(\Omega_{k}\right)$-module $A$ by $A^{\wedge}(k i)$. Then we have the two-sided decomposition

$$
\hat{\Omega}=\bigoplus_{k i}\left(\Omega_{k}\right)^{\wedge(k i)}
$$

where each $\left(\Omega_{k}\right)^{\wedge(k i)}$ is a matrix-local, hence indecomposable ring. Each ring $\left(\Omega_{k}\right)^{\wedge(k i)}$ has a unique indecomposable projective (left) module $U_{k i}$ [LO, 6.6]. If $B_{k}$ denotes the unique indecomposable projective $\Omega_{k}$-module, we have

$$
\hat{B}_{k} \cong \oplus_{i}\left(U_{k i}\right)^{s_{k i}}
$$

where $s_{k i}$ is the splitting number of $M_{k i}^{\prime}[\mathrm{LO}, 6.6]$.

Let $X$ be a $\hat{\Lambda}$-module such that $X_{Q}$ is $\hat{\Lambda}_{Q}$-projective. Then [LO, (6.14.2)] there is an expression:

$$
X_{Q} \cong \bigoplus_{k i}\left(\left(U_{k i}\right)^{\rho_{k i}}\right)_{Q} .
$$

We call $\rho(X)=\left(\left(\rho_{k i}\right)\right)=\left(\left(\rho_{k i}(X)\right)\right)$ the array of ranks of $X$. Note that this array is not a matrix, since its rows can have different lengths. For a given $X$, the array $\rho(X)$ is the same for all splitting orders $\Omega$, because it can be expressed in terms of $\Gamma$ [LO, (6.14.1)] and $\Gamma$ is unique up to conjugacy when $R$ is semilocal [LO, (4.1.7)]. The purpose of this array of ranks is to allow the statement of the following theorem [LO, 6.15]. 
Package Deal Theorem 6.2 (Completions of orders). Let $X$ be a (f.g.) left $\hat{\Lambda}$ module and $\Omega$ a splitting order $(\Lambda \subseteq \Omega \subseteq \Gamma)$. Then the following conditions are equivalent.

(i) $X \cong \hat{A}$ for some (f.g.) $\Lambda$-module $A$.

(ii) $X_{Q}$ is $\hat{\Lambda}_{Q}$-projective and, for every $k$, the array of ranks $\rho(X)=\left(\left(\rho_{k i}\right)\right)$ satisfies

$$
\left(\rho_{k 1}, \rho_{k 2}, \ldots\right) \in \mathbb{N} \cdot\left(s_{k 1}, s_{k 2}, \ldots\right)
$$

(where $\mathbb{N}$ denotes the nonnegative integers).

(iii) $X_{Q} \cong \hat{P}_{Q}$ for some f.g. projective $\Omega$-module $P$.

Remark 6.3. The array of ranks defined above generalizes the array of ranks defined previously, in connection with torsionfree modules. See [LO, 6.23].

Lemma 6.4. Let $X$ be a $\hat{\Lambda}$-module such that $X_{Q}$ is $\hat{\Lambda}$-projective. Then

$$
\rho(X)=0 \Longleftrightarrow X \text { has finite length. }
$$

Proof. The assertion that $\rho(X)=0$ is equivalent to $X_{Q}=0$, which is equivalent to $d X=0$ for some $d \in R-\bigcup Q$. Since $X$ is a finitely generated $\hat{R}$-module and the ring $\hat{R} / \hat{R} d=(R / R d)^{\wedge}=R / R d$ is artinian, $X$ has finite length as an $\hat{R}$-module, hence as a $\hat{\Lambda}$-module.

Conversely, suppose that $X$ has finite length as a $\hat{\Lambda}$-module. Then [LO, 3.7] shows that $X$ has finite length as an $R$-module and is therefore annihilated by a product of maximal ideals of the semilocal ring $R$, hence by some power of $J(R)$. But $J(R)$ contains elements of $R-\bigcup Q$ by [LO, 1.3] (in view of our assumption that $Z(\Lambda)$ has no artinian direct summands). So, as in the first sentence of the proof, $\rho(X)=0$.

Corollary 6.5. Let $X$ be a $\hat{\Lambda}$-module such that $X_{Q}$ is $\hat{\Lambda}_{Q}$-projective. Suppose:

(i) Row 1 of $\rho(X)$ equals $\left(s_{11}, s_{12}, \ldots\right)$ [ $s_{1 i}$ the splitting number of $M_{1 i}^{\prime}$ ];

(ii) All other rows of $\rho(X)$ equal 0 ; and

(iii) No nonzero direct summand of $X$ has finite length.

Then $X$ is an indecomposable package (i.e. the completion of some indecomposable $\Lambda$-module).

Proof. Conditions (i) and (ii) yield $X=\hat{A}$ for some $\Lambda$-module $A$, by Theorem 6.2. Suppose that $A=B \oplus D$. Then $\rho(\hat{A})=\rho(\hat{B})+\rho(\hat{D})$ (coordinatewise sum) with, say, $\rho(\hat{B}) \neq 0$. Theorem 6.2 [see (6.2.1)] then yields $\rho(\hat{D})=0$. By Lemma $6.4, \hat{D}$ has finite length. Condition (iii) then shows that $\hat{D}=0$, hence $D=0$.

The purpose of the next two lemmas is to build some indecomposable $\hat{\Lambda}$-modules for use in the package deal game.

Lemma 6.6. Let $\Omega$ be a splitting order containing $\Lambda$. Suppose that $Z(\Lambda)$ is a local ring and $\hat{\Omega}$ is not an indecomposable ring, so that at least two terms occur in decomposition (6.1.2). Then there exist orthogonal, primitive idempotents $e \in$ $\left(\hat{\Omega}_{1}\right)^{\wedge}(11)$ and $e^{\prime} \in\left(\hat{\Omega}_{k}\right)^{\wedge}(k i)$, for some $(k, i) \neq(1,1)$, such that the $\hat{\Lambda}$-module $\hat{\Lambda}\left(e+e^{\prime}\right)$ has exactly one maximal submodule. 
Proof. Since $Z(\Lambda)$ is a local ring, $\hat{\Lambda}$ is an indecomposable ring [LO, 6.4].

Let $1=\sum_{j} d_{j}$ where the $d_{j}$ are orthogonal, primitive idempotents of $\hat{\Lambda}$, and consider the 2-sided decomposition (6.1.2) of $\hat{\Omega}$. Renumber the $d_{j}$ so that those with nonzero projection in $\left(\Omega_{1}\right)^{\wedge(11)}$ are $d_{1}, \ldots, d_{u}$, and set $d=\sum_{j=1}^{u} d_{j}$, an idempotent of $\hat{\Lambda}$. If $d \in \hat{\Omega}_{1}$, then $d$ is the identity element of $\hat{\Omega}_{1}$, and hence is a central idempotent of $\hat{\Lambda}$. But this contradicts indecomposability of the ring $\hat{\Lambda}$.

Consequently, some $d_{j}$, when written as a sum of orthogonal primitive idempotents of $\hat{\Omega}$, has at least one summand $e \in\left(\Omega_{1}\right)^{\wedge(11)}$ and at least one summand $e^{\prime} \in\left(\hat{\Omega}_{k i}\right)^{\wedge(k i)}$ with $(k, i) \neq(1,1)$. Since complete rings are semiperfect, the indecomposable projective $\hat{\Lambda}$-module $\hat{\Lambda} d_{j}$ has a unique maximal submodule. Therefore so does its homomorphic image $\hat{\Lambda} d_{j}\left(e+e^{\prime}\right)=\hat{\Lambda}\left(e+e^{\prime}\right)$.

Lemma 6.7. Let $\Omega$ be a splitting order $(\Lambda \subseteq \Omega \subseteq \Gamma)$. Suppose that $\hat{\Omega}$ has orthogonal primitive idempotents $e, e^{\prime}$ such that the $\hat{\Lambda}$-module $\Lambda\left(e+e^{\prime}\right)$ has exactly one maximal submodule and $\hat{\Omega} e \cong U_{11}$, the indecomposable projective $\left(\Omega_{1}\right)^{\wedge(11)}$-module. Then there is an indecomposable $\hat{\Lambda}$-module $V$ such that $V_{Q} \cong\left(U_{11}\right)_{Q}$ but $V ¥ U_{11}$.

Proof. We may assume that $R=Z(\Lambda)$. The module $V$ we construct will be a homomorphic image of $\hat{\Lambda}\left(e+e^{\prime}\right)$. Since this last module has a unique maximal submodule, all of its nonzero homomorphic images are indecomposable. So we can forget about indecomposability.

Let $C \subseteq J(\Lambda) \cap J(\Omega)$ be a conductor ideal for $\Lambda$ and $\Omega$, as in (3.5.3). Then $\hat{C}$ is a common ideal of $\hat{\Lambda}$ and $\hat{\Omega}$ such that $\hat{C}_{Q}=\hat{\Lambda}_{Q}=\hat{\Omega}_{Q}$. [As mentioned in Notation 6.1, $Q$ denotes $Q(R)$.] Moreover, $\hat{C} \subseteq J(\hat{\Omega})[\mathrm{LO}, 3.8]$.

Note that $\hat{\Lambda}\left(e+e^{\prime}\right) \supseteq \hat{C} e^{\prime}$ since $\hat{\Lambda}\left(e+e^{\prime}\right) \supseteq \hat{\Lambda} e^{\prime}\left(e+e^{\prime}\right)=\hat{\Lambda} e^{\prime}$. Let $V=$ $\hat{\Lambda}\left(e+e^{\prime}\right) / \hat{C}^{2} e^{\prime}$.

Since $e, e^{\prime}$ are orthogonal idempotents of $\hat{\Omega}$ they are orthogonal idempotents of $\hat{\Omega}_{Q}=\hat{\Lambda}_{Q}$. Therefore

$$
V_{Q}=\left[\hat{\Lambda}\left(e+e^{\prime}\right) / \hat{C}^{2} e^{\prime}\right]_{Q} \cong \frac{\hat{\Lambda}_{Q} e \oplus \hat{\Lambda}_{Q} e^{\prime}}{\hat{\Lambda}_{Q} e^{\prime}} \cong \hat{\Lambda}_{Q} e \cong\left(U_{11}\right)_{Q}
$$

as desired.

Note that $U_{11} \subseteq \hat{\Omega} \subseteq \hat{\Lambda}_{Q}$. So, to prove that $V \nsubseteq U_{11}$, it suffices to prove that $V$ has a nonzero $\hat{\Lambda}$-submodule whose $Q$-localization is zero. (Recall that we have taken $R=Z(\Lambda)$.) We claim that $T=\hat{C} e^{\prime} / \hat{C}^{2} e^{\prime}$ is such a submodule. We have $T \subseteq V$ since, as already shown, $\hat{C} e^{\prime} \subseteq \hat{\Lambda}\left(e+e^{\prime}\right)$. Since $C_{Q}=\Lambda_{Q}$ we have $T_{Q}=0$; and since $\hat{C} \subseteq J(\hat{\Omega})$ we have $T \neq 0$.

Recall that our proof of the necessary and sufficient conditions for KSM was reduced to the case that $Z(\Lambda)$ is local in Theorem 5.6. The proof of our KSM Theorem 1.1 is completed by the following result.

Theorem 6.8 (Local KSM). Suppose that $Z(\Lambda)$ is a local ring and $\Lambda \neq \Gamma$. Then the following conditions are equivalent.

(i) $\Lambda$ satisfies KSM.

(ii) Primitive idempotents of $\Gamma_{k}$ remain primitive modulo $J\left(\Gamma_{k}\right)$ [and therefore every $\Gamma_{k}$ is matrix-local]. 
(iii) Every $\hat{\Lambda}$-module such that $X_{Q}$ is $\Lambda_{Q}$-projective is the $J(R)$-adic completion of some $\Lambda$-module.

Proof. Let $\Omega$ be a splitting $R$-order $(\Lambda \subseteq \Omega \subseteq \Gamma)$. Since $\Lambda \neq \Gamma$ we can choose $\Omega$ such that $\Lambda \neq \Omega$ [LO, 5.2]. Recall that $s_{k i}$ denotes the splitting number of any maximal ideal $M_{k i}^{\prime}$ of $\Gamma_{k}$, and hence of the corresponding maximal ideal $M_{k i}$ of $\Omega$, by [LO, (6.1.2) and (6.1.5)]. Also, primitive idempotents of $\Gamma_{k}$ remain primitive modulo $M_{k i}^{\prime}$ if and only if $s_{k i}=1$, by (3.5.2). To complete the transition between the terminology used in statement (ii) and the terminology to be used in its proof, we note that when $\Gamma_{k}$ is matrix-local we have $J\left(\Gamma_{k}\right)=M_{k 1}$.

(i) $\Rightarrow$ (ii). We show that if some $\Gamma_{k}$ has more than one maximal ideal $M_{k}^{\prime}$ or some splitting number $s_{k i} \neq 1$, then KSM fails.

Assume first that, say, $\Gamma_{1}$, has at least two maximal ideals. Then, $\Omega_{1}$ has at least two maximal ideals $M_{11}, M_{12}$; and therefore $\hat{\Omega}$ has at least two terms in its 2sided decomposition (6.1.2). Hence, by Lemma 6.6 there are orthogonal, primitive idempotents $e \in\left(\hat{\Omega}_{1}\right)^{\wedge(11)}$ and $e^{\prime} \in\left(\hat{\Omega}_{k}\right)^{\wedge(k i)}$, for some $(k, i) \neq(1,1)$, such that the $\hat{\Lambda}$-module $\hat{\Lambda}\left(e+e^{\prime}\right)$ has exactly one maximal submodule. Then, by Lemma 6.7 there is an indecomposable $\hat{\Lambda}$-module $V$ such that $V_{Q} \cong\left(U_{11}\right)_{Q}$ but $V \neq U_{11}$. A second application of this pair of lemmas yields an indecomposable $\hat{\Lambda}$-module $W$ such that $W_{Q} \cong\left(U_{12}\right)_{Q}$ but $W \neq U_{12}$. Consider the following isomorphic direct sums of $\hat{\Lambda}$-modules, where $Y=\bigoplus_{i \geq 3}\left(U_{1 i}\right)^{s_{1 i}}$, and the right-hand side was formed from the left-hand side by interchanging $W$ and $U_{12}$.

$$
\begin{aligned}
{\left[V^{s_{11}} \oplus W^{s_{12}} \oplus Y\right] \oplus\left[\left(U_{11}\right)^{s_{11}} \oplus\left(U_{12}\right)^{s_{12}} \oplus Y\right] } & \\
& \cong\left[V^{s_{11}} \oplus\left(U_{12}\right)^{s_{12}} \oplus Y\right] \oplus\left[\left(U_{11}\right)^{s_{11}} \oplus W^{s_{12}} \oplus Y\right] .
\end{aligned}
$$

By Corollary 6.5 each bracketed term in (6.8.1) is an indecomposable package. Moreover, since KSM holds for $\hat{\Lambda}$, the four bracketed terms are mutually nonisomorphic $\hat{\Lambda}$-modules. Therefore the $\Lambda$-modules whose completions are these bracketed terms yield a counterexample to KSM for $\Lambda$. We may assume, from now on, that every $\Gamma_{k}$ and $\Omega_{k}$ is matrix-local.

Assume next that, say, $s_{11} \neq 1$. Let $X$ be any $\hat{\Lambda}$-module such that $X_{Q}$ is $\hat{\Lambda}_{Q^{-}}$ projective. Since the $\Omega_{k}$ are matrix-local, each row of $\rho(X)$ consists of the single number $\rho_{k 1}(X)$. We consider three cases.

Case $1 . \Omega$ has at least two summands $\Omega_{k}$. Let $V$ be the same $\hat{\Lambda}$-module that was used in (6.8.1). Then, since $s_{11} \geq 2$, the following direct sums of $\hat{\Lambda}$-modules yield, as above, a counterexample to KSM for $\Lambda$ :

$$
\left[V^{s_{11}}\right] \oplus\left[\left(U_{11}\right)^{s_{11}}\right] \cong\left[V \oplus\left(U_{11}\right)^{\left(s_{11}-1\right)}\right] \oplus\left[V^{\left(s_{11}-1\right)} \oplus U_{11}\right] .
$$

Case 2. $\Omega=\Omega_{1}$ and some primitive idempotent $d$ of $\hat{\Lambda}$ does not remain primitive in $\hat{\Omega}$. Say $d=e+e^{\prime}+f$ where $e, e^{\prime}, f$ are orthogonal idempotents of $\hat{\Omega}$ and $e, e^{\prime}$ are primitive. By completeness of $\hat{\Lambda}$, the indecomposable $\hat{\Lambda}$-module $\hat{\Lambda} d$ has a unique maximal submodule, namely $J(\hat{\Lambda}) d$, hence so does its homomorphic image $\hat{\Lambda}\left(e+e^{\prime}\right)$. Thus Lemma 6.7 again yields a $\hat{\Lambda}$-module $V$ that can be used in (6.8.2) to contradict KSM for $\Lambda$.

Case 3. $\Omega=\Omega_{1}$ and all primitive idempotents of $\hat{\Lambda}$ remain primitive in $\hat{\Omega}$. Since $\Lambda \neq \Omega$ we have $\hat{\Lambda} \neq \hat{\Omega}$, and hence there is a primitive idempotent $e$ in $\hat{\Lambda}$ such that $\hat{\Lambda} e \neq \hat{\Omega} e$. We claim that $\hat{\Lambda} e \neq \hat{\Omega} e$ as $\hat{\Lambda}$-modules. 
Let $C \subseteq J(\Lambda) \cap J(\Omega)$ be a conductor ideal for $\Lambda$ and $\Omega$, as in (3.5.3). Then

$$
\hat{\Lambda} e / \hat{C} e \subset \hat{\Omega} e / \hat{C} e
$$

Since $\Lambda / C$ and $\Omega / C$ are $R$-modules of finite length [LO, 3.7], they are their own completions. Hence the two $\hat{\Lambda}$-modules in (6.8.3) have different finite lengths, and are therefore not isomorphic. On the other hand any $\hat{\Lambda}$-isomorphism $\hat{\Lambda} e \cong \hat{\Omega} e$ would take $\hat{C} e$ onto itself, and therefore induce an isomorphism between the modules in (6.8.3). This completes the proof of the claim.

Since $\rho(\hat{\Lambda} e)=\rho(\hat{\Omega} e)$ but $\hat{\Lambda} e \not \hat{\Omega} e$, we can substitute $V=\hat{\Lambda} e$ into (6.8.2) to get a counterexample to KSM for $\Lambda$ [in fact, to TFKS], thus completing the proof of (i) $\Rightarrow$ (ii).

(ii) $\Rightarrow$ (iii) $\Rightarrow$ (i). This is done in [LO, 6.16], under the slightly more general hypothesis that $R$ is semilocal.

Remark 6.9. Before proceeding to the problem of torsionfree KS, in the next several sections, it may be instructive to look back a bit. In the reduction of the proof of KSM to the semilocal case, only torsionfree modules were needed in order to see that "only one singular maximal ideal" is a necessary condition for KSM. In the reduction to the local case, in the present section, we needed four examples in order to establish our necessary conditions, namely (6.8.1) and Cases 1,2,3 of the proof of Theorem 6.8. All but Case 3 used mixed modules in what looks like an essential way. So we find it surprising that there are very few "exceptional" rings $\Lambda$, with $R$ semilocal, that satisfy TFKS but not KSM.

The analysis of TFKS that follows is more intricate than that of KSM in two ways. More complicated modules are needed in order to obtain the conditions necessary for TFKS; and the exceptional rings require complicated matrix arguments in the proof of sufficiency (because indecomposable torsionfree modules over these rings need not remain indecomposable over $\hat{\Lambda}$ ). However the analysis will be simpler in one way: the complicated machinery of completions can be replaced by the simpler device of looking at modules over artinian pairs: the pair of rings that occur in a conductor square for $\Lambda$ and any convenient splitting order $\Omega$.

\section{Linked AND Inertial Maximal Ideals}

Throughout this section $\Xi \subseteq \Upsilon$ are rings with the same 1. As a prelude to proving the TFKS theorem, we examine the maximal ideals of $\Upsilon$ in terms of their contractions to $\Xi$. In the applications, $\Xi \subseteq \Upsilon$ will be one of the following pairs of rings: $\Lambda \subseteq \Gamma, \Lambda \subseteq \Omega, \bar{\Lambda} \subseteq \bar{\Omega}$ or $\hat{\Lambda} \subseteq \hat{\Omega}$. Recall that $R$ is semilocal in this part of the paper, and $C$ denotes a conductor ideal for $\Lambda$ and $\Omega$, as defined above (3.5.3).

Definitions 7.1. Maximal ideals $M$ and $N$ of $\Upsilon$ will be called $\Xi$-linked if $(M \cap \Xi)+(N \cap \Xi) \subset \Xi$ (strict inclusion). Equivalently, $M$ and $N$ are $\Xi$-linked if and only if their intersections with $\Xi$ are contained in some common maximal ideal of $\Xi$. If $\Xi$ and $\Upsilon$ are commutative noetherian domains of Krull dimension 1, with $\Upsilon$ integral over $\Xi$, then $M$ and $N$ are $\Xi$-linked if and only if they lie over a common maximal ideal of $\Xi$.

We say that maximal ideals $M$ and $N$ of $\Upsilon$ are in the same $\Xi$-component of $\operatorname{maxspec}(\Upsilon)$ if there are maximal ideals of $\Upsilon, M=M_{0}, M_{1}, \ldots, M_{n}=N$, such that $M_{i-1}$ is $\Lambda$-linked to $M_{i}$ for $i=1, \ldots, n$. We call maxspec $(\Upsilon) \Xi$-connected if it has only one $\Xi$-component. 
Proposition 7.2. The following statements are equivalent if $C \subseteq J(\Omega)$.

(i) $Z(\Lambda)$ is a local ring (and therefore $\Lambda$ is an indecomposable ring).

(ii) $\hat{\Lambda}$ is an indecomposable ring.

(iii) $\operatorname{maxspec}(\hat{\Omega})$ is $\hat{\Lambda}$-connected.

(iv) $\operatorname{maxspec}(\Omega)$ is $\Lambda$-connected.

(v) maxspec $(\bar{\Omega})$ is $\bar{\Lambda}$-connected.

Proof. Note that, since $C \subseteq J(\Omega)$, we also have $C \subseteq J(\Lambda)$ [LO, 1.4]. Therefore conditions (iv) and (v) are obviously equivalent. (iii) $\Longleftrightarrow$ (iv). The set of ideals $X$ of $\hat{\Lambda}$ such that $\hat{\Lambda} / X$ has finite length is in one-to-one correspondence with the set of ideals $N$ of $\Lambda$ such that $\Lambda / N$ has finite length, via $N \rightarrow X=\hat{R} N$, by [LO, 3.8], and the analogous fact holds for $\Omega$ and $\hat{\Omega}$. Moreover, if $M$ is a maximal ideal of $\Omega$ then $\Omega / M$ is an $\Omega$-module of finite length, hence an $R$-module of finite length [LO, 3.7]. But then $\Lambda /(M \cap \Lambda) \subseteq \Omega / M$ shows that $\Lambda /(M \cap \Lambda)$ is an $R$-module of finite length, hence a $\Lambda$-module of finite length. Now let $M, N$ be maximal ideals of $\Omega$, and $X=\hat{R} M$ and $Y=\hat{R} N$ the corresponding maximal ideals of $\hat{\Omega}$. We will show that $M$ and $N$ are $\Lambda$-linked if and only if $X$ and $Y$ are $\hat{\Lambda}$-linked.

Suppose $(X \cap \hat{\Lambda})+(Y \cap \hat{\Lambda})=\hat{\Lambda}$. Then $\hat{R}[(M \cap \Lambda)+(N \cap \Lambda)]=\hat{\Lambda}(=\hat{R} \Lambda)$ because flatness of $\hat{R}$ as an $R$-module enables it to distribute over the two intersections [Bo '72, Chap. 1, §2.6]. Therefore $(M \cap \Lambda)+(N \cap \Lambda)=\Lambda$. Conversely, $(M \cap$ $\Lambda)+(N \cap \Lambda)=\Lambda$ yields an expression of the form $x+y=1$ with $x \in M \cap \Lambda$ and $y \in N \cap \Lambda$; and this shows that $(X \cap \hat{\Lambda})+(Y \cap \hat{\Lambda})=\hat{\Lambda}$.

(iii) $\Rightarrow$ (ii). Central idempotents of $\hat{\Lambda}$ remain central in $\hat{\Lambda}_{Q}$; and $\hat{\Lambda}_{Q}=\hat{\Omega}_{Q}$. Therefore central idempotents in $\hat{\Lambda}$ remain central in $\hat{\Omega}$. This shows that every ring decomposition of $\hat{\Lambda}$ induces a compatible ring decomposition of $\hat{\Omega}$. So if $\hat{\Lambda}=$ $\hat{\Lambda}_{1} \oplus \hat{\Lambda}_{2}$ every maximal ideal of $\hat{\Omega}$ contains either $\hat{\Lambda}_{1}$ or $\hat{\Lambda}_{2}$. Clearly, maximal ideals containing $\hat{\Lambda}_{1}$ cannot be in the same $\hat{\Lambda}$-component as maximal ideals containing $\hat{\Lambda}_{2}$. Therefore if $\hat{\Lambda}$ decomposes, maxspec $(\hat{\Omega})$ is not $\hat{\Lambda}$-connected.

(ii) $\Rightarrow$ (iii). This is the nontrivial implication. By $[\mathrm{LO}, 6.5], \hat{\Omega}$ is a direct sum of matrix-local rings. For the purposes of the present proof we write this direct sum in the form $\hat{\Omega}=\bigoplus_{\alpha} \hat{\Omega}_{\alpha}$, and note that the unique maximal ideal of each $\hat{\Omega}_{\alpha}$ equals $J\left(\hat{\Omega}_{\alpha}\right)$.

For every $\alpha$ let $M_{\alpha}=J\left(\hat{\Omega}_{\alpha}\right) \oplus\left(\bigoplus_{\gamma \neq \alpha} \hat{\Omega}_{\gamma}\right)$. Then $\left\{M_{\alpha}\right\}$ is the set of maximal ideals of $\hat{\Omega}$. As an induction hypothesis we assume that the first $m$ coordinate rings $\hat{\Omega}_{1}, \ldots, \hat{\Omega}_{m}$ are numbered in such a way that - given any distinct two $\hat{\Omega}_{\beta} \neq \hat{\Omega}_{\gamma}$ of these $m$ rings - it is possible to get from $M_{\beta}$ to $M_{\gamma}$ by means of a sequence of maximal ideals $M_{\alpha}(1 \leq \alpha \leq m)$ such that consecutive terms of this sequence are $\hat{\Lambda}$-linked. This is vacuously true when $m=1$. And if $\bigoplus_{\alpha=1}^{m} \hat{\Omega}_{\alpha}=\hat{\Omega}$ our implication (ii) $\Rightarrow$ (iii) is proved.

Let $\hat{\Omega}^{\prime}=\bigoplus_{\alpha=1}^{m} \hat{\Omega}_{\alpha}$ and $\hat{\Omega}^{\prime \prime}=\bigoplus_{\alpha>m} \hat{\Omega}_{\alpha}$, so that $\hat{\Omega}=\hat{\Omega}^{\prime} \oplus \hat{\Omega}^{\prime \prime}$. We may assume that $\hat{\Omega}^{\prime \prime} \neq 0$.

Let $\hat{\Lambda}^{\prime}, \hat{\Lambda}^{\prime \prime}$ be the projections of $\hat{\Lambda}$ in $\hat{\Omega}^{\prime}, \hat{\Omega}^{\prime \prime}$ respectively, and note that $\hat{\Lambda}^{\prime}$ and $\hat{\Lambda}^{\prime \prime}$ are nonzero. Then $\hat{\Lambda}$ is a subdirect sum of $\hat{\Lambda}^{\prime} \oplus \hat{\Lambda}^{\prime \prime}$. Therefore [L '81, 3.2] there exist, for some ring $W$, surjective ring homomorphisms $f^{\prime}: \hat{\Lambda}^{\prime} \rightarrow W$ and $f^{\prime \prime}: \hat{\Lambda}^{\prime \prime} \rightarrow W$ such that $\hat{\Lambda}$ equals the pullback

$$
\hat{\Lambda}=\left\{\left(x^{\prime}, x^{\prime \prime}\right) \in \hat{\Lambda}^{\prime} \oplus \hat{\Lambda}^{\prime \prime} \mid f^{\prime}\left(x^{\prime}\right)=f^{\prime \prime}\left(x^{\prime \prime}\right)\right\} .
$$


Since $\hat{\Lambda}$ is indecomposable, it does not equal the direct sum of $\hat{\Lambda}^{\prime}$ and $\hat{\Lambda}^{\prime \prime}$, and therefore $W \neq 0$. So $W$ has a maximal ideal $\bar{A}$. Let $A^{\prime}, A^{\prime \prime}$ be the inverse images of $\bar{A}$ in $\hat{\Lambda}^{\prime}, \hat{\Lambda}^{\prime \prime}$ respectively. Then $A=\left\{\left(a^{\prime}, a^{\prime \prime}\right) \mid f^{\prime}\left(a^{\prime}\right)=f^{\prime \prime}\left(a^{\prime \prime}\right)\right\}$ is a maximal ideal of $\hat{\Lambda}$ (because $\hat{\Lambda} / A \cong W / \bar{A}$ ). By the next lemma (with the present $\hat{R}$ playing the role of $S$ in that lemma) there exist maximal ideals $N^{\prime}, N^{\prime \prime}$ of $\hat{\Omega}^{\prime}, \hat{\Omega}^{\prime \prime}$ respectively such that $N^{\prime} \cap \hat{\Lambda}^{\prime} \subseteq A^{\prime}$ and $N^{\prime \prime} \cap \hat{\Lambda}^{\prime \prime} \subseteq A^{\prime \prime}$.

We have $N^{\prime} \oplus\left(\bigoplus_{\alpha>m} \hat{\Omega}_{\alpha}\right)=M_{\beta}$ for some $\beta \leq m$ and $\left(\bigoplus_{\alpha=1}^{m} \hat{\Omega}_{\alpha}\right) \oplus N^{\prime \prime}=M_{\gamma}$ for some $\gamma>m$. Moreover, $M_{\beta}$ is $\hat{\Lambda}$-linked to $M_{\gamma}$ since the intersections $M_{\beta} \cap \hat{\Lambda}$ and $M_{\gamma} \cap \hat{\Lambda}$ are contained in $A$. Therefore renumbering so that $\gamma=m+1$ completes the induction.

(i) $\Longleftrightarrow$ (ii). This is proved in [LO, 6.4].

Lemma 7.3 (Weak lying-over lemma). Let $\Xi, \Upsilon$ be module-finite $S$-algebras such that $\Xi \subseteq \Upsilon \subseteq \Xi_{Q(S)}$, where $S$ is any semilocal commutative noetherian ring of dimension 1 and $\Xi_{Q(S)}$ denotes the localization of $\Xi$ at the union of the finite number of minimal primes of $S$. Then for any maximal ideal $A$ of $\Xi$ there is a maximal ideal $N$ of $\Upsilon$ such that $N \cap \Xi \subseteq A$.

Proof. We can assume that $S$ has no artinian ring-direct summands. As in the proof of [LO, 1.4], we can find a common ideal $C$ contained in $J(\Xi)$ and $J(\Upsilon)$, such that $\Xi / C$ and $\Upsilon / C$ are artinian rings. Then, working $\bmod C$, we can assume that $\Xi$ and $\Upsilon$ are artinian rings.

Since $J(\Upsilon) \cap \Xi$ is a nilpotent ideal of $\Xi$, we have $J(\Upsilon) \cap \Xi \subseteq J(\Xi) \subseteq A$. Let $N_{1}, \ldots, N_{k}$ be the maximal ideals of $\Upsilon$. Then the product

$$
\begin{aligned}
\left(N_{1} \cap \Xi\right) \cdots\left(N_{k} \cap \Xi\right) & \subseteq\left(N_{1} \cap \Xi\right) \cap \cdots \cap\left(N_{k} \cap \Xi\right) \\
& =J(\Upsilon) \cap \Xi \\
& \subseteq A .
\end{aligned}
$$

Since maximal ideals are prime, we have $N_{i} \cap \Xi \subseteq A$ for some $N_{i}$, as desired.

Example 7.4. In Proposition 7.2, one cannot add an additional, equivalent statement (i) $)^{\prime} \bar{\Lambda}$ is an indecomposable ring. [The details of this also provide a simple example of a central idempotent of $\bar{\Lambda}$ that cannot be lifted to a central idempotent of $\hat{\Lambda}$.]

Let $\Omega$ be a maximal order that is a domain with local center, and suppose that the unique maximal ideal $M$ of $\Omega$ has splitting number 2. Setting $\bar{\Omega}=\Omega / M$ we have $\bar{\Omega}=M_{2}(\Delta)$ for some division ring $\Delta$. Let $\bar{\Lambda}$ be the subring of $\bar{\Omega}$ consisting of all diagonal matrices, and then let $\Lambda$ be the pullback of conductor square (3.5.4). Note that $\bar{\Lambda}$ is the direct sum of two division rings.

On the other hand, we claim that $\hat{\Lambda}$ is an indecomposable ring. As mentioned in the proof of (ii) $\Rightarrow$ (i), in Proposition 7.2, central idempotents of $\hat{\Lambda}$ remain central in $\hat{\Omega}$. Therefore it suffices to prove that $\hat{\Omega}$ is an indecomposable ring. This is done in [LO, Lemma 6.5].

We call a maximal ideal $M$ of $\Upsilon$ completely inertial over $\Xi$ if, for every $k \geq 1$, the canonical monomorphism $\Xi /\left(M^{k} \cap \Xi\right) \longrightarrow \Upsilon / M^{k}$ is a surjection, hence an isomorphism. In particular, $M \cap \Xi$ is a maximal ideal of $\Xi$. An equivalent definition of "completely inertial" is that $\Xi+M^{k}=\Upsilon$ for all $k \geq 1$.

Proposition 7.5. Let $C$ be any conductor ideal for $\Lambda$ and $\Omega$, and let $M$ be a maximal ideal of $\Omega$ containing $C$. Then the following statements are equivalent. 
(i) $M$ is completely inertial over $\Lambda$.

(ii) $\bar{M}$ is completely inertial over $\bar{\Lambda}$.

(iii) $\Lambda+M^{k}=\Omega$ for some $k$ such that $\bar{M}^{k}=\bar{M}^{k+1}$.

(iv) There is a surjective $\bar{\Lambda}$-homomorphism $\bar{\Lambda} \rightarrow\left(\bar{B}_{M}\right)^{u}$, where $\bar{B}_{M}$ is the unique indecomposable projective left $\bar{\Omega}$-module such that $\bar{M} \bar{B}_{M} \neq \bar{B}_{M}$, and $u$ is the multiplicity of $\bar{B}_{M}$ as a direct summand of $\bar{\Omega} \bar{\Omega}$.

Proof. (i) $\Longleftrightarrow$ (ii) $\Longleftrightarrow$ (iii). Since $\bar{\Omega}$ is an artinian ring, there exists a positive integer $k$ such that $\bar{M}^{k}=\bar{M}^{k+1}$ (hence $\left.=\bar{M}^{k+2}=\cdots\right)$. Therefore $M^{h}+C=$ $M^{k}+C$ for all positive integers $h \geq k$. Now suppose that (iii) holds. Then for every positive integer $h \geq k$ we have

$$
\Lambda+M^{h}+C=\Lambda+M^{k}+C=\Omega .
$$

Since $\Lambda \supseteq C$ we can delete $C$ in the expressions above, showing that $\Lambda+M^{h}=\Omega$ for all $h \geq k$, hence for all $h$. Thus (i) holds. The implication (i) $\Rightarrow$ (ii) is obvious; and (ii) $\Rightarrow$ (iii) is easy if - as in the proof of (iii) $\Rightarrow$ (i) - one makes use of $C$ to pass from $\bar{\Lambda}$ to $\Lambda$, and then deletes $C$ at the end because it is contained in $\Lambda$.

(ii) $\Longleftrightarrow$ (iv). Since $\Omega$ is a splitting order, $\bar{\Omega}$ is a direct sum of artinian matrixlocal rings $[\mathrm{LO},(6.18 .1)]$. Artinian matrix-local rings have only one simple left module, hence only one indecomposable projective left module. Therefore we have decompositions

$$
\bar{\Omega}=\bar{\Omega}^{\prime} \oplus \bar{\Omega}^{\prime \prime}, \quad \bar{M}=J\left(\bar{\Omega}^{\prime}\right) \oplus \bar{\Omega}^{\prime \prime}, \quad \bar{\Omega}^{\prime} \cong\left(\bar{B}_{M}\right)^{u}
$$

where $\bar{\Omega}^{\prime}$ is a matrix-local ring, and the first two decompositions are 2-sided decompositions. Letting $k$ be the index of nilpotence of $J\left(\bar{\Omega}^{\prime}\right)$ yields $\bar{M}^{k}=\bar{M}^{k+1}=\bar{\Omega}^{\prime \prime}$ and therefore $\bar{\Omega}^{\prime} \cong \bar{\Omega} / \bar{M}^{k}$. Therefore, in the statement of (iv), we can replace $\left(\bar{B}_{M}\right)^{u}$ by $\bar{\Omega} / \bar{M}^{k}$. If (ii) holds it is clear that $\bar{\Lambda}$ maps onto $\bar{\Omega} / \bar{M}^{k}$; that is, (iv) holds.

Conversely, if (iv) holds, then $\bar{\Lambda}$ can be mapped onto the $\bar{\Lambda}$-module $\bar{\Omega} / \bar{M}^{k}$. Since $\bar{M}$ is a 2 -sided ideal of $\bar{\Omega}$, the kernel of any such map contains $\bar{M}^{k} \cap \bar{\Lambda}$, and therefore the composition length of the $\bar{\Lambda}$-module $\bar{\Lambda} /\left(\bar{M}^{k} \cap \bar{\Lambda}\right)$ is equal to or greater than the $\bar{\Lambda}$-length of $\bar{\Omega} / \bar{M}^{k}$. It follows that the natural imbedding $\bar{\Lambda} /\left(\bar{M}^{k} \cap \bar{\Lambda}\right) \rightarrow \bar{\Omega} / \bar{M}^{k}$ is a surjection. Therefore (iii), hence (ii), holds.

Lemma 7.6. Suppose that every maximal ideal of every splitting order $\Omega(\Lambda \subseteq$ $\Omega \subseteq \Gamma)$ is completely inertial over $\Lambda$. Then $\Gamma$ is module-finite over $R$ and every such $\Omega$ equals $\Gamma$.

Proof. Let $\Lambda \subseteq \Omega^{\prime \prime} \subseteq \Omega \subseteq \Gamma$, with $\Omega^{\prime \prime}, \Omega$ splitting orders. Note that every maximal ideal of $\Omega$ is completely inertial over $\Omega^{\prime \prime}$. Therefore we can assume that $\Lambda$ is itself a splitting order. Our next goal is to prove that $\Lambda=\Omega$.

Choose a conductor ideal $C$ for $\Lambda$ and $\Omega$ that is contained in $J(\Lambda) \cap J(\Omega)$. It suffices to prove that $\bar{\Lambda}=\bar{\Omega}$. Note [Proposition 7.5] that every maximal ideal of $\bar{\Omega}=\Omega / C$ is completely inertial over $\bar{\Lambda}=\Lambda / C$. Now, $\bar{\Omega}$ is a direct sum of artinian matrix-local rings, by [LO, (6.18.1)]. This also holds for $\bar{\Lambda}$ since we are assuming that $\Lambda$ is a splitting order. Moreover, the maximal ideals of $\bar{\Lambda}$ are contractions of those of $\bar{\Omega}$, since the maximal ideals of all splitting orders are the contractions of those of $\Gamma[\mathrm{LO}, 5.3]$. Therefore set-theoretic inclusion provides a one-to-one correspondence between the matrix-local summands of $\bar{\Lambda}$ and those of $\bar{\Omega}$; and so we can suppose that $\bar{\Lambda}$ and $\bar{\Omega}$ are artinian matrix-local rings. In this situation the maximal ideal of $\bar{\Omega}$ equals $J(\bar{\Omega})$. The natural map $\bar{\Lambda} \rightarrow \bar{\Omega} / J(\bar{\Omega})^{k}$ is a surjection for 
every $k$; and choosing $k$ to be the index of nilpotence of $J(\bar{\Omega})$ shows that $\bar{\Lambda}=\bar{\Omega}$, as desired.

Finally, we show (still assuming that $\Lambda$ is a splitting order) that $\Lambda=\Gamma$. If not, then there is a splitting order $\Omega$ containing $\Lambda$ and some element of $\Gamma-\Lambda$ [LO, 5.2]. But this contradicts the equality $\Lambda=\Omega$ proved above.

\section{Three Indecomposable $(\bar{\Lambda}, \bar{\Omega})$-Modules}

The primary objective of this section is to construct three indecomposable modules over the artinian pair $(\bar{\Lambda}, \bar{\Omega})$, for use when playing the package deal game in the proof of the TFKS theorem in the next section. We make extensive use of the definitions of " $\Lambda$-linked" and "completely inertial" maximal ideals, introduced in the previous section.

Notation 8.1. Throughout this section $R$ remains semilocal and $\Omega$ is a splitting $R$-order $(\Lambda \subseteq \Omega \subseteq \Gamma)$; and $\bar{\Lambda}=\Lambda / C$ and $\bar{\Omega}=\Omega / C$ are computed with respect to a conductor ideal $C$ such that $C \subseteq J(\Lambda) \cap J(\Omega)$.

The artinian ring $\bar{\Omega}$ is a direct sum of matrix-local rings, $\bar{\Omega}=\bigoplus_{k i} \bar{\Omega}_{k i}$ (3.5.9), [LO, (6.18.1)]. In our applications of the results of the present section we need to index the indecomposable one- and two-sided summands of $\bar{\Omega}$ by the maximal ideals of $\Omega$.

Let $M$ be a maximal ideal of $\Omega$ and $\bar{M}$ its image in $\bar{\Omega}$. Then let $\bar{\Omega}_{M}$ be the unique $\bar{\Omega}_{k i}$ such that the projection of $\bar{M}$ in $\bar{\Omega}_{k i}$ is a maximal ideal of $\bar{\Omega}_{k i}$. The matrix-local ring $\bar{\Omega}_{M}$ has a unique projective (left) module that we call $\bar{B}_{M}$. (It is called $\bar{B}_{k i}$ in [LO, 6.19]). As a left module, $\bar{\Omega}_{M}$ is the direct sum of some number $u(M)$ of copies of $\bar{B}_{M}$. Thus we have:

$$
\bar{\Omega}=\bigoplus_{M \in \operatorname{maxspec}(\Omega)} \bar{\Omega}_{M} \quad\left[\bar{\Omega}_{M} \cong\left(\bar{B}_{M}\right)^{u(M)}\right] .
$$

We observe that, for maximal ideals $M, N$ of $\Omega$ :

$$
M \bar{B}_{N}=\bar{B}_{N} \quad \Longleftrightarrow \quad M \neq N .
$$

This holds since the projection of the maximal ideal $\bar{M}$ in the matrix-local ring $\bar{\Omega}_{M}$ equals $J\left(\bar{\Omega}_{M}\right)$, while the projection of $\bar{M}$ in every other $\bar{\Omega}_{N}$ is the whole ring $\bar{\Omega}_{N}$.

Lemma 8.2. Let $N$ and $M$ be maximal ideals of $\Omega$ that are $\Lambda$-linked, and such that $N$ is completely inertial over $\Lambda$. Then:

(i) $u(M) \geq u(N)$.

(ii) Suppose that $u(M)=u(N)$. Then $\bar{N} \cap \bar{\Lambda}$ is the unique maximal ideal of $\bar{\Lambda}$ containing $\bar{M} \cap \bar{\Lambda}$.

Proof. For any artinian ring $A$, let $e(A)$ denote the number of terms that result when one expresses $1_{A}$ as a sum of orthogonal primitive idempotents. If $A^{\prime}$ is an artinian subring of $A$ we have $e\left(A^{\prime}\right) \leq e(A)$. Since idempotents can be lifted modulo nil ideals, and $J(A)$ contains no nonzero idempotents, we have $e(A / J(A))=e(A)$. In fact, $e(A)$ is the composition length of $A / J(A)$.

For any surjective ring homomorphism $A \rightarrow B$, we have $e(A) \geq e(B)$, since the given homomorphism induces a surjective ring homomorphism $A / J(A) \rightarrow B / J(B)$. Moreover, $e(A)=e(B)$ implies that $A$ and $B$ become isomorphic modulo their radicals. 
Proof of (i). It follows from (8.1.2) and (8.1.1) that the multiplicity of $\bar{B}_{M}$ in the decomposition of $\bar{\Omega}$ equals $e(\bar{\Omega} / \bar{M})$, and a similar statement holds for $N$. Therefore we can restate the conclusion of the statement (i) in the form $e(\bar{\Omega} / \bar{M}) \geq e(\bar{\Omega} / \bar{N})$. We prove this by establishing the following chain of statements:

$$
e(\bar{\Omega} / \bar{M}) \geq e(\bar{\Lambda} /(\bar{M} \cap \bar{\Lambda})) \geq e(\bar{\Lambda} /(\bar{N} \cap \bar{\Lambda}))=e(\bar{\Omega} / \bar{N}) .
$$

Since $N$ is completely inertial over $\Lambda$, its image $\bar{N}$ is completely inertial over $\bar{\Lambda}$; and this proves the equality at the end of the chain. The inequality at the beginning of the chain holds since $\bar{\Omega} / \bar{M} \supseteq \bar{\Lambda} /(\bar{M} \cap \bar{\Lambda})$.

Since $\bar{M}$ and $\bar{N}$ are $\bar{\Lambda}$-linked, the ideals $\bar{M} \cap \bar{\Lambda}$ and $\bar{N} \cap \bar{\Lambda}$ are contained in some common maximal ideal of $\bar{\Lambda}$. But, since $\bar{N}$ is completely inertial over $\bar{\Lambda}, N \cap \bar{\Lambda}$ is itself a maximal ideal of $\bar{\Lambda}$. Consequently $\bar{M} \cap \bar{\Lambda} \subseteq \bar{N} \cap \bar{\Lambda}$; and so there is a surjective ring homomorphism $\bar{\Lambda} /(\bar{M} \cap \bar{\Lambda}) \rightarrow \bar{\Lambda} /(\bar{N} \cap \bar{\Lambda})$. This yields the inequality in the middle of the chain.

Proof of (ii). In the previous paragraph we showed that $\bar{N} \cap \bar{\Lambda}$ is a maximal ideal of $\bar{\Lambda}$ and contains $\bar{M} \cap \bar{\Lambda}$; and the surjection near the end of the previous paragraph induces a surjection of those rings modulo their Jacobson radicals. It therefore suffices to show that this last surjection is an isomorphism.

The first and last terms in (8.2.1) equal $u(M)$ and $u(N)$, respectively, and are equal by the hypothesis of statement (ii). Therefore the middle two terms are equal; and this establishes the desired isomorphism.

Notation 8.3. Recall, from Notation 3.5, that a (left) module over the artinian $\operatorname{pair}(\bar{\Lambda}, \bar{\Omega})$ is an inclusion of (f.g.) modules $[X \subseteq Y]$, where $Y$ is a projective $\bar{\Omega}$ module and $X$ is a $\bar{\Lambda}$-submodule such that $\bar{\Omega} X=Y$. Since $Y$ is $\bar{\Omega}$-projective, there are (unique) non-negative integers ("ranks") $\rho(M)(M \in \operatorname{maxspec}(\Omega))$ such that

$$
Y \cong \bigoplus_{M}\left(\bar{B}_{M}\right)^{\rho(M)} .
$$

Lemma 8.4. If $\bar{\Lambda}=X \oplus X^{\prime}$ as $\bar{\Lambda}$-modules, then $\bar{\Omega}=\bar{\Omega} X \oplus \bar{\Omega} X^{\prime}$ as $\bar{\Omega}$-modules.

Proof. We have $X=\bar{\Lambda} e$ and $X^{\prime}=\bar{\Lambda}(1-e)$ for some $e=e^{2} \in \bar{\Lambda}$. Therefore $\bar{\Omega} X=\bar{\Omega} e$ and $\bar{\Omega} X^{\prime}=\bar{\Omega}(1-e)$.

Lemma 8.5. Let $\left[P \subseteq Y \oplus Y^{\prime}\right]$ be a $(\bar{\Lambda}, \bar{\Omega})$-module with ${ }_{\bar{\Lambda}} P$ indecomposable projective and $Y \neq 0$. Then $\left[\pi_{Y}(P) \subseteq Y\right]$ is indecomposable, where $\pi_{Y}$ is the projection map to $Y$.

Proof. Note that $\bar{\Omega} \cdot \pi_{Y}(P)=Y$, so $\left[\pi_{Y}(P) \subseteq Y\right]$ is a $(\bar{\Lambda}, \bar{\Omega})$-module. Since $P$ has exactly one maximal submodule, every nonzero homomorphic image of $P$, in particular $\pi_{Y}(P)$, is indecomposable. Consequently, the $(\bar{\Lambda}, \bar{\Omega})$-module $\left[\pi_{Y}(P) \subseteq\right.$ $Y]$ is also indecomposable.

The next three propositions construct our promised indecomposable $(\bar{\Lambda}, \bar{\Omega})$ modules.

Proposition 8.6. Let $M$ and $N$ be distinct maximal ideals of $\Omega$ which are $\Lambda$-linked. Then there is an indecomposable $(\bar{\Lambda}, \bar{\Omega})$-module $\left[X \subset \bar{B}_{M} \oplus \bar{B}_{N}\right]$ (strict inclusion) such that $X$ has exactly one maximal submodule. 
Proof. Strictness of the inclusion is immediate, once the rest of the proposition is proved, because $\left[\bar{B}_{M} \oplus \bar{B}_{N} \subseteq \bar{B}_{M} \oplus \bar{B}_{N}\right]$ is obviously decomposable. By combining terms we can rewrite (8.1.1) in the form

$$
\bar{\Omega}=\bar{\Omega}_{M} \oplus \bar{\Omega}_{N} \oplus \bar{\Omega}_{C}
$$

It suffices, by Lemmas 8.4 and 8.5, to show that $\bar{\Lambda} \bar{\Lambda}$ has an indecomposable summand with non-zero projection in both $\bar{\Omega}_{M}$ and $\bar{\Omega}_{N}$. So suppose not.

Then every indecomposable summand of $\overline{ }_{\Lambda} \bar{\Lambda}$ has nonzero projection in at most one of $\bar{\Omega}_{M}, \bar{\Omega}_{N}$. Consequently, we can write $\overline{\bar{\Lambda}} \bar{\Lambda}=X_{M} \oplus X_{N}$, where $X_{M} \subseteq \bar{\Omega}_{M} \oplus \bar{\Omega}_{C}$ and $X_{N} \subseteq \bar{\Omega}_{N} \oplus \bar{\Omega}_{C}$. By (8.1.2), $\bar{\Omega}_{M} \oplus \bar{\Omega}_{C}=\bar{N} \bar{\Omega}_{M} \oplus \bar{N} \bar{\Omega}_{C} \subseteq \bar{N}$. Therefore $X_{M} \subseteq \bar{N}$. Similarly, $X_{N} \subseteq \bar{M}$. So

$$
\bar{\Lambda}=X_{M} \oplus X_{N} \subseteq(\bar{N} \cap \bar{\Lambda})+(\bar{M} \cap \bar{\Lambda}) .
$$

Since $\bar{M}$ and $\bar{N}$ are $\bar{\Lambda}$-linked, the right-hand side, above, is properly contained in $\bar{\Lambda}$; and this contradiction completes the proof.

Proposition 8.7. Let $M$ be a maximal ideal of $\Omega$ such that $\bar{M}$ is not completely inertial over $\bar{\Lambda}$. Then there is an indecomposable $(\bar{\Lambda}, \bar{\Omega})$-module $\left[X \subset \bar{B}_{M}\right]$ or $\left[X \subset\left(\bar{B}_{M}\right)^{2}\right]$ (proper inclusion) such that $X$ has exactly one maximal submodule.

Proof. By combining terms we can rewrite (8.1.1) in the form

$$
\bar{\Omega}=\bar{\Omega}_{M} \oplus \bar{\Omega}_{C} \quad\left(\bar{\Omega}_{M} \cong \bar{B}_{M}^{u}\right)
$$

Let $e$ be the central idempotent of $\bar{\Omega}$ such that $\bar{\Omega}_{M}=\bar{\Omega} e$. Then $\bar{\Lambda} e \neq \bar{\Omega} e$, by Proposition 7.5 (ii) and (iv).

Consider the $(\bar{\Lambda}, \bar{\Omega})$-module $[\bar{\Lambda} e \subset \bar{\Omega} e]$, and note that its structure as a $(\bar{\Lambda}, \bar{\Omega})$ module is the same as its structure as a $(\bar{\Lambda} e, \bar{\Omega} e)$-module. Lemma 8.4 yields an indecomposable $(\bar{\Lambda} e, \bar{\Omega} e)$-module, so $(\bar{\Lambda}, \bar{\Omega})$-module, of the form $[X \subset Y]$, where $X$ is an indecomposable direct summand of $\bar{\Lambda} e$, and therefore has exactly one maximal submodule. Since $Y$ is a direct summand of $\bar{\Omega} e \cong \bar{B}_{M}^{u}$, we have $Y=\bar{B}_{M}^{s}$ for some $s$. If $s=1$ or 2 we are done. Otherwise projection into the first two coordinates yields the indecomposable $(\bar{\Lambda}, \bar{\Omega})$-module $\left[\pi(X) \subseteq \bar{B}_{M}^{2}\right]$, by Lemma 8.5. The inclusion in this last $(\bar{\Lambda}, \bar{\Omega})$-module must be strict, since $\left[\bar{B}_{M}^{2} \subseteq \bar{B}_{M}^{2}\right]$ obviously decomposes.

Remarks 8.8 (Projective covers). We review the properties of projective covers of (finitely generated) modules over artinian rings, in terminology needed for the next proof. Let $A$ be a left artinian ring. In this context, a projective cover of an $A$-module $U$ can be defined to be a surjective $A$-module homomorphism $f: P \rightarrow U$ such that $\operatorname{ker}(f) \subseteq J(A) \cdot P$. Sometimes one also calls the module $P$ a projective cover of $U$. See [Ba '60] for the following facts (in somewhat different terminology).

(i) Every f.g. $A$-module $U$ has a projective cover. (ii) (Uniqueness of the projective cover). If $f^{\prime}: P^{\prime} \rightarrow U$ is another projective cover of $U$, then there is an $A$ module isomorphism $\theta: P \cong P^{\prime}$ such that $f^{\prime} \theta=f$. (iii) If $g: Q \rightarrow U$ is an $A$-module surjection, with $Q$ projective, then there is a decomposition $Q=Q^{\prime} \oplus Q_{0}$ such that the restriction of $g$ to $Q^{\prime}$ is a projective cover and $g\left(Q_{0}\right)=0$. (iv) If $f_{i}: P_{i} \rightarrow U_{i}$ is a projective cover, for $i=1,2, \ldots, n$, then so is $\bigoplus_{i} f_{i}: \bigoplus_{i} P_{i} \rightarrow \bigoplus_{i} U_{i}$.

Remarks 8.9 (Lifting idempotents). Let $f: A \rightarrow \bar{A}$ be any surjective ring homomorphism ( $A$ still left artinian). Then any orthogonal idempotents $\bar{e}_{1}, \ldots, \bar{e}_{n} \in \bar{A}$ can be lifted to orthogonal idempotents $e_{1}, \ldots, e_{n} \in A$ such that $f\left(e_{i}\right)=\bar{e}_{i}$. 
Note that, in the previous paragraph, we did not assume that $\operatorname{ker}(f) \subseteq J(A)$. Since we shall need this slightly sharper-than-usual form of the theorem, and it seems to be missing from many standard texts, we briefly sketch its proof. After introducing one more idempotent, if necessary, we can assume that $1_{\bar{A}}=\sum_{i} \bar{e}_{i}$. This yields a decomposition $\bar{A}=\bigoplus_{i} \bar{A}_{\bar{e}}$ which can be viewed as a decomposition of the $A$-module $\bar{A}$. For each $i$, let $P_{i} \rightarrow \bar{A} \bar{e}_{i}$ be a projective cover of the $A$ module $\bar{A} \bar{e}_{i}$. Then $P=\bigoplus_{i} P_{i} \rightarrow \bar{A}=\bigoplus_{i} \bar{A} \bar{e}_{i}$ is a projective cover, by (iv) above. The natural homomorphism $A \rightarrow \bar{A}$ is also surjection. Therefore, by properties of the projective cover, we have $P$ isormorphic to a direct summand of $A$; and so we may assume that $A=P \oplus Q$ for some complement $Q \subseteq \operatorname{ker}(f)$. Write $1_{A}=e_{1}+\ldots+e_{n}+e^{\prime}$ with each $e_{i} \in P_{i}$ and $e^{\prime} \in Q$. Then the $e_{i}$ are orthogonal idempotents of $A$. Moreover, $1_{\bar{A}}=f\left(1_{A}\right)=\sum_{i} f\left(e_{i}\right)$, with each $f\left(e_{i}\right) \in \bar{A} \bar{e}_{i}$. Uniqueness of expressions in direct sums therefore implies that each $f\left(e_{i}\right)=\bar{e}_{i}$.

We shall also need the following consequence of the above material.

Lemma 8.10. Let $f: A \rightarrow \bar{A}$ be a surjective ring homomorphism, with $A$ left artinian, and let $d, d^{\prime}$ be primitive idempotents of $A$.

(i) If $f(d)$ is nonzero, then it is a primitive idempotent of $\bar{A}$.

(ii) If $\bar{A} f(d) \cong \bar{A} f\left(d^{\prime}\right) \neq 0$ as $\bar{A}$-modules, then $A d \cong A d^{\prime}$ as $A$-modules.

Proof. (i). Since $d$ is a primitive idempotent of the artinian ring $A$, the $A$-module $A d$ has a unique maximal submodule, namely $J(A) d$. Therefore every nonzero homomorphic image of $A d$ is indecomposable. Statement (i) follows, since indecomposability of $\bar{A} f(d)$ implies that the idempotent $f(d)$ is primitive.

(ii). Since $A d$ is an indecomposable $A$-module and $\bar{A} f(d) \neq 0$, it follows from property (iii) of projective covers that the map $f: A d \rightarrow \bar{A} f(d)$ is a projective cover. Statement (ii) now follows from uniqueness of projective covers.

Proposition 8.11. Suppose that $\Omega$ has maximal ideals $N, M$ such that:

(i) $N$ and $M$ are $\Lambda$-linked;

(ii) $N$ is completely inertial over $\bar{\Lambda}$ but $M$ is not; and

(iii) $u(N)=u(M)$. (See (8.1.1). $)$

Then there is an indecomposable $(\bar{\Lambda}, \bar{\Omega})$-module $\left[Z \subset \bar{B}_{N}^{2} \oplus \bar{B}_{M}\right]$ with proper inclusion.

Proof. This is the most intricate of our three constructions. By combining terms we can rewrite (8.1.1) in the form

$$
\bar{\Omega}=\bar{\Omega} e_{N} \oplus \bar{\Omega} e_{M} \oplus \bar{\Omega}_{C} \quad\left(\bar{\Omega} e_{N} \cong \bar{B}_{N}^{u}, \bar{\Omega} e_{M} \cong \bar{B}_{M}^{u}\right)
$$

where $e_{N}$ and $e_{M}$ are central idempotents of $\bar{\Omega}, u=u(M)=u(N)$, and neither $\bar{B}_{N}$ nor $\bar{B}_{M}$ is isomorphic to a direct summand of $\bar{\Omega}_{C}$. Note that $\bar{N} \cap \bar{\Lambda}$ is a maximal ideal of $\bar{\Lambda}$, by (ii).

Claim 1. There is a $\bar{\Lambda}$-module decomposition

$$
\bar{\Lambda} \cong(\bar{\Lambda} d)^{u} \oplus P
$$

where $u$ is as in (8.11.1) and $d$ is a primitive idempotent of $\bar{\Lambda}$ that remains primitive modulo $\bar{N} \cap \bar{\Lambda}$. To see this, note first that, since $\bar{N}$ is completely inertial over $\bar{\Lambda}$, we have $\Lambda /(\bar{N} \cap \bar{\Lambda}) \cong \bar{\Omega} / \bar{N}$ as rings. This shows that the simple artinian ring $\bar{A}=\bar{\Lambda} /(\bar{N} \cap \bar{\Lambda})$ is the direct sum of $u$ mutually isomorphic simple modules. This, together with (8.11.1), yields $u$ orthogonal primitive idempotents $\bar{d}_{i}$ of $\bar{A}$. Since 
the artinian ring $\bar{A}$ is simple, we have $\bar{A} \bar{d}_{i} \cong \bar{A}_{j}$ for every $i, j$. These idempotents can be lifted to $u$ orthogonal idempotents $d_{i}$ of $\bar{\Lambda}$, by Remarks 8.9. Note that any expression of the form $d_{i}=a+b$, where $a$ and $b$ are primitive idempotents of $\bar{\Lambda}$, yields $f(a)=0$ or $f(b)=0$ (because $\bar{d}_{i}$ is a primitive idempotent). Therefore, after writing each $d_{i}$ as a sum of orthogonal primitive idempotents of $\bar{\Lambda}$, discarding some terms, and changing notation, we can assume that each $d_{i}$ is a primitive idempotent. Then $\bar{\Lambda} d_{i} \cong \bar{\Lambda} d_{i}$ for every $i, j$, by Lemma 8.10(ii), and this completes the proof of the claim.

Claim 2. We have

$$
\bar{\Omega} d \cong \bar{B}_{N} \oplus \bar{B}_{M} \oplus V
$$

where neither $\bar{B}_{M}$ nor $\bar{B}_{N}$ is isomorphic to a direct summand of $V$.

First we show that $\bar{B}_{N}$ is isomorphic to a direct summand of $\bar{\Omega} d$. By (8.1.2) and (8.1.1) it suffices to show that $d$ remains nonzero in $\bar{\Omega} / \bar{N}$. But $\bar{\Lambda} /(\bar{N} \cap \bar{\Lambda}) \subseteq \Omega / \bar{N}$, and part of Claim 1 was that $d$ remains nonzero in $\bar{\Lambda} /(\bar{N} \cap \bar{\Lambda})$.

Similarly, to show that $\bar{B}_{M}$ is isomorphic to a direct summand of $\bar{\Omega} d$ it suffices to show that $d$ remains nonzero in $\bar{\Lambda} /(\bar{M} \cap \bar{\Lambda})$. Since $\bar{M}$ and $\bar{N}$ are $\bar{\Lambda}$-linked, $\bar{M} \cap \bar{\Lambda}$ and $\bar{N} \cap \bar{\Lambda}$ are contained in a common maximal ideal of $\bar{\Lambda}$. But, since $\bar{N}$ is completely inertial over $\bar{\Lambda}, \bar{N} \cap \bar{\Lambda}$ is a maximal ideal of $\bar{\Lambda}$. Hence $\bar{M} \cap \bar{\Lambda} \subseteq \bar{N} \cap \bar{\Lambda}$. Since $d$ remains nonzero modulo the larger of these ideals, it also remains nonzero modulo the smaller, as desired.

It therefore remains to be shown that neither $\bar{B}_{N}$ nor $\bar{B}_{M}$ is isomorphic to a direct summand of the complementary summand $V$ in (8.11.3). To do this, tensor expression (8.11.2) over $\bar{\Lambda}$ with $\bar{\Omega}$ and substitute (8.11.3) into the resulting expression, getting

$$
\bar{\Omega} \cong\left(\bar{B}_{N} \oplus \bar{B}_{M} \oplus V\right)^{u} \oplus \bar{\Omega} P .
$$

Since the multiplicity of each of $\bar{B}_{N}$ and $\bar{B}_{M}$ as a direct summand of $\bar{\Omega}$ is $u$, as displayed in (8.11.1), we see that $V$ is as claimed, and complete the proof of Claim 2. It also follows from this multiplicity argument that $\bar{B}_{M}$ is not isomorphic to a direct summand of $\bar{\Omega} P$, and therefore

$$
e_{M}(\bar{\Omega} P)=0 .
$$

For the rest of this proof we consider the isomorphism in (8.11.3) to be equality. Since $\bar{\Lambda} d \subseteq \bar{\Omega} d$ we therefore have $e_{M} \bar{\Lambda} d \subseteq \bar{B}_{M}$. This inclusion must be proper. For otherwise we would have $\left(e_{M} \bar{\Lambda} d\right)^{u}=\bar{B}_{M}^{u}$, and so there would be a $\bar{\Lambda}$-module surjection of $\bar{\Lambda}$ onto $\left(\bar{B}_{M}\right)^{u}$. It would then follow from Proposition 7.5 that $M$ is completely inertial over $\Lambda$, a contradiction. Letting $W=\bar{\Lambda} d$, we now have:

(8.11.6) $W=\bar{\Lambda} d \subset \bar{B}_{N} \oplus \bar{B}_{M} \oplus V, \quad \bar{\Omega} W=\bar{B}_{N} \oplus \bar{B}_{M} \oplus V, \quad$ and $e_{M} W \subset \bar{B}_{M}$.

In particular, $\left[W \subset \bar{B}_{N} \oplus \bar{B}_{M} \oplus V\right]$ is a $(\bar{\Lambda}, \bar{\Omega})$-module, and is indecomposable because $W$ is an indecomposable $\bar{\Lambda}$-module. This is the starting point for our construction of the $(\bar{\Lambda}, \bar{\Omega})$-module described in the statement of the proposition.

Claim 3. There is a nonzero $\bar{\Lambda}$-homomorphism $\bar{\theta}: W \rightarrow B^{\prime}:=\bar{B}_{M} / e_{M} W$. Since $B^{\prime} \neq 0$ by (8.11.6), there is a nonzero homomorphism $\bar{\theta}: \bar{\Lambda} \rightarrow B^{\prime}$. It therefore suffices to show that the $\bar{\Lambda}$-module $P$ in (8.11.2) satisfies $\operatorname{Hom}_{\bar{\Lambda}}\left(P, B^{\prime}\right)=0$. Since $P$ is a direct sum of indecomposable modules of the form $\bar{\Lambda} c$ (with $c=c^{2}$ ) that are not isomorphic to $W$, it suffices to show that $\operatorname{Hom}_{\bar{\Lambda}}\left(\bar{\Lambda} c, B^{\prime}\right)=0$. Since $\bar{\Lambda} c$ is projective, any nonzero homomorphism $\bar{\eta}: \bar{\Lambda} c \rightarrow B^{\prime}$ lifts to a nonzero homomorphism 
$\eta: \bar{\Lambda} c \rightarrow \bar{B}_{M}$. Thus there exists $x \in \bar{\Lambda}$ such that $0 \neq \eta(x c)=x c \eta(c) \in \bar{B}_{M}$. Since multiplication by the central idempotent $e_{M}$ equals the identity on $\bar{B}_{M}$, we have $0 \neq e_{M} x c \in e_{M} P$. This contradiction of (8.11.5) proves Claim 3.

Let $\theta: W \rightarrow \bar{B}_{M}$ be a lift of the map $\bar{\theta}$ in Claim 3, and define a $\bar{\Lambda}$-homomorphism $\alpha: W^{2} \rightarrow \bar{B}_{N}^{2} \oplus \bar{B}_{M}$ by

$$
\alpha\left(w_{1}, w_{2}\right)=\left(e_{N} w_{1}, e_{N} w_{2}, e_{M} w_{1}+\theta\left(w_{2}\right)\right) .
$$

Let $Z=\operatorname{im}(\alpha)$. We claim that $L=\left[Z \subset \bar{B}_{N}^{2} \oplus \bar{B}_{M}\right]$ is the desired indecomposable $(\bar{\Lambda}, \bar{\Omega})$-module.

To see that $L$ is a $(\bar{\Lambda}, \bar{\Omega})$-module we must show that $\bar{\Omega} Z=\bar{B}_{N}^{2} \oplus \bar{B}_{M}$. We have $\bar{\Omega} W \supseteq e_{N} \bar{\Omega} W \oplus e_{M} \bar{\Omega} W=\bar{B}_{N} \oplus \bar{B}_{M}$ and $e_{N} \bar{\Omega} W=\bar{B}_{N}$ by (8.11.6). Let $(x, y, z) \in \bar{B}_{N}^{2} \oplus \bar{B}_{M}$. The second equality in the previous sentence shows that we can find an element of the form $(0, y, ?)$ in $\bar{\Omega} \cdot \alpha(0, W)$. The first equality in that sentence then shows that we can add an appropriate element of $\bar{\Omega} \cdot \alpha(W, 0)$ to this to get $(x, y, z)$.

Claim 4. There is no surjective $\bar{\Lambda}$-module map: $e_{M} W \rightarrow e_{M} Z$. Note that there is a $\bar{\Lambda}$-module surjection: $Z \rightarrow e_{M} W+\theta(W) \supset e_{M} W$, obtained from (8.11.7) by mapping $Z$ to the third coordinate of $\alpha\left(W^{2}\right)$. The inclusion is proper by Claim 3 . Since multiplication by $e_{M}$ is the identity map on $\bar{B}_{M}$, there is therefore a surjective $\bar{\Lambda}$-homomorphism of $e_{M} Z$ onto a module properly containing $e_{M} W$. Hence, if Claim 4 were false, there would be a $\bar{\Lambda}$-surjection of $e_{M} W$ onto a module properly containing $e_{M} W$, which is impossible since $W$ has finite length.

Claim 5. The $\bar{\Lambda}$-module map $\alpha: W^{2} \rightarrow Z=\operatorname{im}(\alpha)$ induced by (8.11.7) is a projective cover. If not, then by property (iii) of projective covers we could write $W^{2}$ as the direct sum of two nonzero modules, one of which $\alpha$ maps onto $Z$ (and the other of which $\alpha$ maps to zero). By KS for modules of finite length, it would therefore be possible to map $W$ onto $Z$, contrary to Claim 4 .

Finally, we show that $L$ is indecomposable. Any nontrivial decomposition must have the form

$$
L=\left[Z \subset \bar{B}_{N}^{2} \oplus \bar{B}_{M}\right]=\left[Z_{1} \subseteq Y \oplus \bar{B}_{M}\right] \oplus\left[Z_{2} \subseteq Y^{\prime}\right] \quad\left(Y \oplus Y^{\prime} \cong \bar{B}_{N}^{2}\right)
$$

Each of $Z_{1}$ and $Z_{2}$ has a projective cover $P_{i} \rightarrow Z_{i}$. The direct sum $P \rightarrow Z$ of these projective covers is a projective cover of $Z$, and therefore $P \cong W^{2}$ by Claim 5 and uniqueness of the projective cover. Therefore each $P_{i} \cong W$; and therefore there exists a $\bar{\Lambda}$-module surjection: $W \rightarrow Z_{1}$, and hence a surjection: $e_{M} W \rightarrow e_{M} Z_{1}$. Since $e_{M} Y^{\prime} \subseteq e_{M} \bar{B}_{N}^{2}=0$, multiplying decomposition (8.11.8) by $e_{M}$ shows that $e_{M} Z=e_{M} Z_{1}$. Therefore $e_{M} W$ can be mapped onto $e_{M} Z$. This contradiction of Claim 4 completes the proof.

\section{Necessary Conditions for Local TFKS}

Notation 9.1. The main result of this section, Theorem 9.6, completes the proof of our necessary conditions for TFKS by proving them when $Z(\Lambda)$ is local. (But see $\S 11$ for the explicit description of the second exceptional class of rings satisfying TFKS.)

Recall that $\Gamma$ denotes a normalization of $\Lambda$, as in [LO, $\S 4$, unless otherwise specified. Recall also that a uniserial module is a module whose lattice of submodules is totally ordered by inclusion. As usual $R$ remains semilocal. 
Lemma 9.2. Suppose $\Gamma$ is matrix-local with maximal ideal $M$, and $\Gamma$ is modulefinite over $R$. Let $U \neq 0$ be a uniserial left $\Gamma$-module of finite length $\lambda$. Then statements (i) and (ii) are equivalent.

(i) Every $\Gamma$-generator of $U$ is a $\Lambda$-generator. (That is, every element of $U$ that generates $U$ as a $\Gamma$-module also generates $U$ as a $\Lambda$-module.)

(ii) The unique simple left $\Gamma$-module $S=U / M U$ remains simple as a $\Lambda$-module and, if $\lambda \geq 2, \Gamma(M \cap \Lambda) \Gamma=M$.

When these conditions hold, $\Lambda$ is matrix-local with maximal ideal $J(\Lambda)=M \cap \Lambda$.

Proof. We claim that if $S$, the simple $\Gamma$-module, remains simple as a $\Lambda$-module then $M \cap \Lambda$ is a maximal ideal of $\Lambda$. Since the ring $\Gamma / M$ is simple artinian with simple left module $S$, we have $\Gamma / M \cong S^{n}$ as $\Gamma$-modules, for some $n$. Therefore $\operatorname{ann}_{\Gamma} S=\operatorname{ann}_{\Gamma} \Gamma / M=M$ (left annihilators); and so we have $\operatorname{ann}_{\Lambda} S=M \cap \Lambda$. Therefore if the faithful module $S$ over the artinian ring $\Lambda /(M \cap \Lambda)$ is simple, then the ring $\Lambda /(M \cap \Lambda)$ is simple, as claimed.

Note that, since $\Gamma$ is matrix-local with maximal ideal $M=J(\Gamma)$, the unique $\Gamma$-composition series of $U$ is $U \supset M U \supseteq M^{2} U \supseteq \cdots$ (and all composition factors are $\cong S)$.

(i) $\Rightarrow$ (ii). The generator condition in (i) passes down to all homomorphic images of $U$, since all proper $\Gamma$-submodules of $U$ are contained in the unique maximal $\Gamma$ submodule of $U$. The simple $\Gamma$-module $S$ is a homomorphic image of $U$ and is generated by each of its nonzero elements. Therefore, by (i), the $\Lambda$-module $S$ is generated by each of its nonzero elements, and is therefore simple. It follows from the first paragraph of this proof that $M \cap \Lambda$ is a maximal ideal of $\Lambda$, as desired.

In proving the second statement of (ii) we may assume that $\lambda \geq 2$. Note that the generators of the $\Gamma$-module $U / M^{2} U$ of length 2 are the elements not in its unique maximal $\Gamma$-submodule $M U / M^{2} U$. Every nonzero ideal of the matrix-local maximal order $\Gamma$ is a power of $M$. Therefore, if $\Gamma(M \cap \Lambda) \Gamma=M$ does not hold we have $\Gamma(M \cap \Lambda) \Gamma \subseteq M^{2}$. Therefore the module $U / M^{2} U$ over the artinian ring $\Lambda /(M \cap \Lambda)$ (a simple ring, by the previous paragraph), is semisimple; hence its submodule $M U / M^{2} U$ is a direct summand of it. Any complement of this direct summand consists of $\Gamma$-generators that are not $\Lambda$-generators, contradicting the fact that the generator condition in (i) passes down to $U / M^{2} U$.

(ii) $\Rightarrow$ (i). Since the composition factors of ${ }_{\Gamma} U$ all equal $S$, and since $S$ is assumed $\Lambda$-simple, $U$ also has composition length $\lambda$ as a $\Lambda$-module. To complete the proof of (i) it suffices to show that the unique $\Gamma$-composition series of $U$ is also its unique $\Lambda$ composition series. For then the set of generators of $U$, over either ring, is $U-M U$. We prove this by induction on $\lambda$.

For $\lambda=1$ there is nothing further to prove, since simple modules are uniserial. For $\lambda=2, U$ is either $\Lambda$-semisimple (necessarily the direct sum of two copies of $S)$ or $\Lambda$-uniserial. If the first possibility holds, then $(M \cap \Lambda) U=0$ and therefore $M U=\Gamma(M \cap \Lambda) \Gamma U=0$; so we reach the contradiction that $\lambda=1$.

Now assume that $\lambda \geq 3$. Let $U=U_{\lambda} \supset U_{\lambda-1} \supset \cdots \supset U_{1} \supset U_{0}=0$ be the unique $\Gamma$-composition series of $U$, and assume that we have proved the claim for all uniserial left $\Gamma$-modules of length $<\lambda$.

Let $A$ be any proper $\Lambda$-submodule of $U$. Then by our induction hypothesis, applied to $U / U_{1}$, we have $A+U_{1}=U_{i}$ for some $i \geq 1$. If $i<\lambda$, then the induction hypothesis, applied to $U_{i}$, shows that $A$ equals some $U_{j}$, as desired. Therefore we may assume that $i=\lambda$; that is, $A+U_{1}=U$. We show that this leads to a 
contradiction. We have $A \neq U_{\lambda-1}$ since $A+U_{1}=U$. In fact, $A \nsupseteq U_{\lambda-1}$ because $U_{\lambda-1}$ is a maximal $\Lambda$-submodule of $U$ and $A$ is a proper submodule of $U$. Moreover, $U_{\lambda-1}=\left(A \cap U_{\lambda-1}\right)+U_{1}$ since $A+U_{1}=U$. Therefore $U_{\lambda-1}$ is the sum of two properly smaller $\Lambda$-submodules, and hence is not $\Lambda$-uniserial. Since the composition length of $U_{\lambda-1}$ over $\Gamma$ is $\lambda-1$, this contradicts our induction hypothesis, and completes the proof of (i).

To prove the supplementary statement we need to show that $M \cap \Lambda$ is the only maximal ideal of $\Lambda$. Since $M \cap \Lambda$ is a maximal ideal of $\Lambda$ and $M$ is the unique maximal ideal of $\Gamma$, this follows from the weak lying-over Lemma 7.3.

Definition 9.3. Let $f, g: \Gamma \rightarrow \Psi$ be ring homomorphisms. The equalizer eq $(\Gamma, f, g)$ is defined to be

$$
\mathrm{eq}(\Gamma, f, g)=\{x \in \Gamma \mid f(x)=g(x)\}
$$

Lemma 9.4. Suppose that $\Gamma$ is module-finite over $R$ and has exactly two maximal ideals $M, N$, both of which are completely inertial over $\Lambda$, and that $Z(\Lambda)$ is a local ring. Then $\Lambda$ is an equalizer $\Lambda=\mathrm{eq}(\Gamma, f, g)$ for some surjective $f, g$ and some positive integer $h$ such that $\operatorname{ker}(f)=M^{h}$ and $\operatorname{ker}(g)=N^{h}$.

Proof. Since $\Gamma$ is module-finite over $R$, there is a conductor ideal $C$ for $\Lambda$ and $\Gamma$, as defined above (3.5.3), and $\Lambda$ is the pullback of its conductor square, as in (3.5.4):

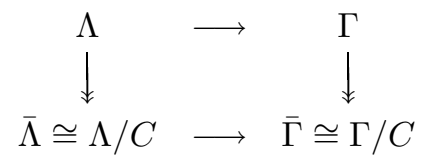

We claim that $C \subseteq J(\Gamma)(=M \cap N)$. Consider the $J(R)$-adic completions $\hat{\Lambda} \subseteq \hat{\Gamma}$ and their common ideal $\hat{C}$. Note that $Z(\hat{\Lambda})=Z(\Lambda)^{\wedge}[\mathrm{LO}, 6.3]$, and therefore $Z(\hat{\Lambda})$ is a local ring. We may therefore suppose that $\Lambda$ and $\Gamma$ are themselves complete (see [LO, 3.8] for more details). Since the classical complete, maximal order $\Gamma$ has exactly two maximal ideals, it is the direct sum of two maximal orders in simple algebras $\Gamma=\Gamma_{M} \oplus \Gamma_{N}$, and $M=M^{\prime} \oplus \Gamma_{N}$ where $M^{\prime}$ is the unique maximal ideal of $\Gamma_{M}$. Now suppose that $C \nsubseteq M \cap N$. Then (say) $C \nsubseteq M$. Since $C$ is the direct sum of its projections in $\Gamma_{M}$ and $\Gamma_{N}$ we conclude that its projection $\Gamma_{M} C$ is not contained in the unique maximal ideal $M^{\prime}$ of $\Gamma_{M}$, and therefore $\Gamma_{M} C=\Gamma_{M}$. Since $\Gamma_{M} C \subseteq C$ we conclude that $C$ contains the identity element $e$ of $\Gamma_{M}$. But $C \subseteq \Lambda$, and therefore $e \in \Lambda$. Therefore $\Lambda$ contains two orthogonal central idempotents $e$ and $1-e$, contradicting the fact that $Z(\Lambda)$ is local and proving the claim. (We now drop the extra, completeness assumption used in this paragraph.)

In the rest of this proof we choose $C$ to be the largest possible conductor for $\Lambda$ and $\Gamma$. Consequently, $\bar{\Lambda}$ and $\bar{\Gamma}$ have no common nonzero ideal.

Now $\Gamma$ is a splitting order since it is module-finite over $R$. So we can apply [LO, (6.18.1)] with $\Gamma$ in place of $\Omega$ to conclude that $\bar{\Gamma}$ is a direct sum of matrix-local rings, and the bottom line of conductor square (9.4.1) has the form

$$
\bar{\Lambda} \rightarrow \bar{\Gamma}=\bar{\Gamma}_{M} \oplus \bar{\Gamma}_{N}
$$

where $\bar{\Gamma}_{M}$ and $\bar{\Gamma}_{N}$ are artinian matrix-local rings. The maximal ideals of $\bar{\Gamma}$ are then $\bar{M}=J\left(\bar{\Gamma}_{M}\right) \oplus \bar{\Gamma}_{N}$ and $\bar{N}=\bar{\Gamma}_{M} \oplus J\left(\bar{\Gamma}_{N}\right)$. Let $\pi_{M}$ and $\pi_{N}$ be the coordinate projection maps in (9.4.2), and $i: \bar{\Lambda} \rightarrow \bar{\Gamma}$ the inclusion map. We claim that the compositions $\pi_{M} i$ and $\pi_{N} i$ are isomorphisms. 
To see that they are surjections, let $k$ be any integer greater than or equal to the indices of nilpotence of $J\left(\bar{\Gamma}_{M}\right)$ and $J\left(\bar{\Gamma}_{N}\right)$. Since $\bar{M}$ is completely inertial over $\bar{\Lambda}$ we have $\bar{\Lambda}+\bar{M}^{k}=\bar{\Gamma}$. Since $\bar{M}^{k}=\bar{\Gamma}_{N}$ we see that $\pi_{M} i$ is a surjection. Similar reasoning applies to $\pi_{N} i$.

Since $\pi_{N} i: \bar{\Lambda} \rightarrow \bar{\Gamma}_{N}$ is a surjection, the ideal $\operatorname{ker}\left(\pi_{M} i\right)$ of $\bar{\Lambda}$ is also an ideal of $\bar{\Gamma}$, and therefore (by our choice of conductor $C$ ) equals zero. Similarly $\operatorname{ker}\left(\pi_{N} i\right)=0$, completing the proof of the claim.

Let $\nu$ be the natural surjection $\Gamma \rightarrow \bar{\Gamma}$. Then $f=\left(\pi_{M} i\right)^{-1} \pi_{M} \nu$ and $g=$ $\left(\pi_{N} i\right)^{-1} \pi_{N} \nu$ are surjections $\Gamma \rightarrow \bar{\Lambda}$; and since $\Lambda$ is the pullback of its conductor square (9.4.1) we have $\Lambda=\mathrm{eq}(\Gamma, f, g)$, as stated in the lemma.

Thus it remains to be shown that $\operatorname{ker}(f)=M^{h}$ and $\operatorname{ker}(g)=N^{h}$ for some $h$. By the multiplicative ideal theory of maximal orders [R '75, (22.10)], every ideal contained in $M$ but not in $N$ equals some unique power of $M$. Therefore $\operatorname{ker}(f)=M^{a}$, and similarly $\operatorname{ker}(g)=N^{b}$ for positive integers $a, b$. Moreover, in the present situation, $a$ and $b$ are the indices of nilpotence of $J(\bar{\Lambda})$, and therefore $a=b$.

Lemma 9.5. Suppose that $\Gamma$ is a maximal $R$-order in the simple artinian ring $\Gamma_{Q}$, and has exactly two maximal ideals $M, N$. Let $n$ be the integer such that $\Gamma \cong M_{n}(\Delta)$ for some integral domain $\Delta$ (see $[\mathrm{LO},(4.1 .8)])$, and let $\Lambda=\mathrm{eq}(\Gamma, f, g)$ for some $f, g$ where $\operatorname{ker}(f)=M^{h}$ and $\operatorname{ker}(g)=N^{h}$ for some surjective $f, g$ and positive $h$. Then

(i) $\Lambda$ is a matrix-local ring with maximal ideal $J(\Lambda)=M \cap \Lambda=N \cap \Lambda$.

(ii) $\Lambda \cong M_{n}(D)$ for some $D \subset \Delta$.

(iii) $\Delta$ has exactly two maximal ideals $M^{\prime}, N^{\prime}$, and $D=\mathrm{eq}\left(\Delta, f^{\prime}, g^{\prime}\right)$ for some surjective $f^{\prime}, g^{\prime}$ where $\operatorname{ker}\left(f^{\prime}\right)=\left(M^{\prime}\right)^{h}$ and $\operatorname{ker}\left(g^{\prime}\right)=\left(N^{\prime}\right)^{h}$.

Proof. (i). The ideal $C=M^{h} \cap N^{h}$ is the largest conductor ideal for $\Lambda$ and $\Gamma$, and yields a conductor square of the form (9.4.1). The bottom line of this square has the form (9.4.2), both induced maps $\bar{\Lambda} \rightarrow \bar{\Gamma}_{M}$ and $\bar{\Lambda} \rightarrow \bar{\Gamma}_{N}$ are isomorphisms, and we may regard each of $f$ and $g$ as mapping $\Gamma$ onto $\bar{\Lambda}$. Note that $C \subseteq J(\Gamma)$. Therefore, by (3.5.3), $C \subseteq J(\Lambda)$. Statement (i) now follows easily by working modulo $C$.

(ii) Recall that a ring $\Upsilon$ is an $n \times n$ matrix ring if and only if $\Upsilon$ contains a set of $n \times n$ matrix units; that is, elements $e_{i j}(i, j=1,2, \ldots, n)$ such that $e_{i j} e_{j k}=e_{i k}$, $e_{i j} e_{k l}=0$ when $j \neq k$, and $\sum_{i} e_{i i}=1$. Let $e_{i j}$ be the standard $(i, j)$-matrix unit of $\Gamma$, that is, the matrix with 1 in entry $(i, j)$ and zero elsewhere. If every $f\left(e_{i j}\right)=g\left(e_{i j}\right)$, then $e_{i j} \in \Lambda$, and (ii) is obvious. Our proof consists of finding matrix units $d_{i j} \in \Gamma$ with these desired properties. First we observe:

(9.5.1) Suppose that there is an expression $1=\sum_{i=1}^{n} d_{i i}$ where the $d_{i i}$ are orthogonal idempotents in some ring $\Upsilon$, and that $\Upsilon d_{i i} \cong \Upsilon d_{j j}$ as $\Upsilon$-modules, for every $i, j$. Then the $d_{i i}$ are part of a set of $n \times n$ matrix units $d_{i j}$ of $\Upsilon$.

To obtain the off-diagonal matrix units $d_{i j}(i \neq j)$, let them be elements, right multiplications by which yield appropriate isomorphisms $\Upsilon d_{i i} \cong \Upsilon d_{j j}$.

Next let $\psi: \Gamma \rightarrow \Psi$ be any surjective ring homomorphism. We claim that every unit $\bar{u}$ in $\Psi$ can be lifted, via $\psi$, to a unit of $\Gamma$. Let $x$ be any element of $\Gamma$ such that $g(x)=\bar{u}$. Then, since $\bar{u}$ is a unit, there is an expression $a x+b k=1$ with $k \in \operatorname{ker}(\psi)$ and $a, b \in \Gamma$. Since $\Gamma$ is a module-finite algebra over the semilocal $\operatorname{ring} R, \Gamma$ has 1 in its stable range (see, e.g., [W '80, 2.2]) and therefore there exists $c \in \Gamma$ such that $x+c k=u$ is a unit in $\Gamma$. This $u$ clearly lifts $\bar{u}$. 
Now return to the standard matrix units $e_{i j} \in \Gamma$. The elements $f\left(e_{i j}\right)$ form a set of $n \times n$ matrix units of $\bar{\Lambda}$, and so do the elements $g\left(e_{i j}\right)$. This yields two $\bar{\Lambda}$-module decompositions

$$
\bar{\Lambda}=\bigoplus_{i} \bar{\Lambda} f\left(e_{i i}\right)=\bigoplus_{i} \bar{\Lambda} g\left(e_{i i}\right)
$$

in which $\bar{\Lambda} f\left(e_{i i}\right) \cong \bar{\Lambda} f\left(e_{j j}\right)$ for every $i$ and $j$, and an analogous statement holds with $g$ in place of $f$. Since the ring $\bar{\Lambda}$ is artinian we have $\bar{\Lambda} f\left(e_{i i}\right) \cong \bar{\Lambda} g\left(e_{i i}\right)$ for all $i$. The direct sum of $n$ such isomorphisms is an automorphism of the left $\bar{\Lambda}$ module $\bar{\Lambda}$, and therefore equals right multiplication by some unit $\bar{u}$ of $\bar{\Lambda}$. Therefore $\bar{u}^{-1} f\left(e_{i i}\right) \bar{u}=g\left(e_{i i}\right)$. [Projection to the $i^{\text {th }}$ summand in the two decompositions equals right multiplication by $f\left(e_{i i}\right)$ and $g\left(e_{i i}\right)$ respectively. The first projection can be transformed into the second by conjugation with the isomorphism that transforms one decomposition to the other.]

Since $\operatorname{ker}(f)$ and $\operatorname{ker}(g)$ are comaximal, the ring homomorphism $(f, g): \Gamma \rightarrow \bar{\Lambda}^{2}$ is surjective. Therefore the unit $(1, \bar{u}) \in \bar{\Lambda}^{2}$ can be lifted, via $(f, g)$, to a unit $u \in \Gamma$. Then the elements $d_{i i}=u e_{i i} u^{-1}$ are a set of $n$ orthogonal idempotents of $\Gamma$ that belong to $\Lambda=\mathrm{eq}(\Gamma, f, g)$, and whose sum equals 1 . Also, $\Lambda=\bigoplus_{i} \Lambda d_{i i}$.

We claim that $\Lambda d_{i i} \cong \Lambda d_{j j}$ for every $i$ and $j$. By statement (i), the map $\Lambda \rightarrow \bar{\Lambda}$ is a projective cover [see Remarks 8.8], and therefore so is each restricted map $\Lambda d_{i i} \rightarrow \bar{\Lambda} f\left(d_{i i}\right)$. We have $\bar{\Lambda} f\left(d_{i i}\right) \cong \bar{\Lambda} f\left(d_{j j}\right)$ for every $i, j$ since the $f\left(d_{i i}\right)$ are part of a set of $n \times n$ matrix units of $\bar{\Lambda}$. Therefore, by uniqueness of the projective cover, the $\Lambda$-isomorphisms $\bar{\Lambda} f\left(d_{i i}\right) \cong \bar{\Lambda} f\left(d_{j j}\right)$ can be lifted to $\Lambda$-isomorphisms $\Lambda d_{i i} \cong \Lambda d_{j j}$, as claimed.

It follows from (9.5.1) that the orthogonal idempotents $d_{i i} \in \Lambda$ are part of a set of $n \times n$ matrix units $d_{i j}$ of $\Lambda$. These $d_{i j}$ are therefore a set of $n \times n$ matrix units of any ring containing $\Lambda$; in particular, of $\Gamma$. Therefore $\Lambda \cong M_{n}(D)$ and $\Gamma \cong M_{n}\left(\Delta^{\prime}\right)$ where $D=d_{11} \Lambda d_{11} \subseteq \Delta^{\prime}=d_{11} \Gamma d_{11}$. Therefore (ii) is proved as soon as we show that $\Delta^{\prime} \cong \Delta$. But the ring $\Delta$ such that $\Gamma \cong M_{n}(\Delta)$ is unique up to isomorphism since it is the ring of endomorphisms of the unique indecomposable projective left $\Gamma$-module - unique since $\Gamma$ is a maximal $R$-order and $R$ is semilocal. See [LO, (4.1.8)].

(iii). This follows easily from (ii).

Theorem 9.6 (Local Necessity). Suppose $\Lambda$ satisfies TFKS, Z $(\Lambda)$ is a local ring, and $\Lambda \neq \Gamma$. Then one of the following conditions must be satisfied:

(i) Each $\Gamma_{k}$ is matrix-local and its maximal ideal has splitting number 1 (i.e. $\Lambda$ satisfies KSM).

(ii) $\Gamma$ is module-finite over $R ; \Lambda=M_{n}\left(\Lambda^{\prime}\right)$ and $\Gamma=M_{n}\left(\Gamma^{\prime}\right)$ for some $n$, where $\Lambda^{\prime} \subset \Gamma^{\prime}$ and $\Gamma^{\prime}$ is a PID; and one of the following situations holds.

(a) $\Gamma^{\prime}$ has exactly two maximal ideals $M, N$, and $\Gamma^{\prime} / M$ and $\Gamma^{\prime} / N$ are division rings. Moreover, $\Lambda^{\prime}$ is an equalizer $\Lambda^{\prime}=\mathrm{eq}\left(\Gamma^{\prime}, f, g\right)$ for some surjective $f, g$ such that $\operatorname{ker}(f)=M^{h}$ and $\operatorname{ker}(g)=N^{h}$ for some $h \geq 1$.

(b) (abstract version). $\Gamma^{\prime}$ has exactly one maximal ideal $M$, and $M$ has splitting number 2; $\Gamma^{\prime}\left(M \cap \Lambda^{\prime}\right) \Gamma^{\prime}=M$ and the simple left $\Gamma^{\prime}$-module remains simple as a $\Lambda^{\prime}$-module.

The following additional properties of the exceptional rings in (ii)(a) and (ii)(b) are proved in subsection 9.9. 
Supplement 9.7 (to Local Necessity Theorem 9.6). Let $\Gamma^{\prime}$ be a maximal $R$-order in the simple artinian ring $\left(\Gamma^{\prime}\right)_{Q}$.

(a) If $\Lambda^{\prime}$ is as in paragraph (ii)(a) of Theorem 9.6, then $J\left(\Lambda^{\prime}\right)=M \cap \Lambda^{\prime}=N^{\prime} \cap \Lambda$.

(b) If $\Lambda^{\prime}$ is as in paragraph (ii)(b) of Theorem 9.6, then $J\left(\Lambda^{\prime}\right)=M \cap \Lambda^{\prime}$.

In both situations $\Lambda^{\prime}$ and $Z\left(\Lambda^{\prime}\right)$ are local rings.

Proof 9.8 (of Theorem 9.6). This proof makes extensive use of the material on modules over artinian pairs and ranks introduced in in (3.5.4)-(3.5.11) and [LO, 6.18ff]. In particular, $\Omega$ denotes an arbitrarily chosen splitting order $(\Lambda \subseteq \Omega \subseteq \Gamma)$ unless a special choice of $\Omega$ is indicated, and $C$ denotes a conductor ideal for $\Lambda$ and $\Omega$ such that $C \subseteq J(\Lambda) \cap J(\Omega)$, as in (3.5.3). Since $Z(\Lambda)$ is local, maxspec $(\Gamma)$ is $\Lambda$-connected [Proposition 7.2]. Recall that the set of maximal ideals of $\Gamma$ is in one-to-one correspondence with that of $\Omega$ via contraction [LO, 5.3].

Our proof requires one additional bit of notation. Consider the decomposition $\Omega=\bigoplus_{k} \Omega_{k}$, and let $M_{k i}$ be a maximal ideal of $\Omega_{k}$ for some $k$. We define the maximal ideal of $\Omega$ associated with $M_{k i}$ to be

$$
M^{k i}=M_{k i} \oplus\left(\bigoplus_{h \neq k} \Omega_{h}\right) .
$$

In addition to supposing that $\Lambda$ satisfies TFKS and $\Lambda \neq \Gamma$, we may also suppose that $\Lambda$ does not satisfy condition (i) of Theorem 9.6, and then we must show that $\Lambda$ is one of the two exceptional types of rings described in the theorem. We break the proof into two cases, according to whether or not $\Gamma$ is matrix-local.

Case 1. $\Gamma$, hence $\Omega$, has at least two maximal ideals. We show that the situation in statement (ii)(a) of the theorem holds.

Recall the decomposition $\bar{\Omega}_{k}=\bigoplus_{i} \bar{\Omega}_{k i}$ in (3.5.9) We invoke Fundamental Combinatorial Lemma 4.3, with $\mathcal{S}$ the collection of all torsionfree $\Lambda$-modules $L$ such that $\Omega L$ is $\Omega$-projective. The lemma states that, for each $k$, the $\bar{\Omega}_{k i}$ can be renumbered in such a way that the following conditions hold for all indecomposable $(\bar{\Lambda}, \bar{\Omega})$-modules $\left[X \subseteq \bigoplus_{k i}\left(\bar{B}_{k i}\right)^{\rho_{k i}}\right]$ with strict inclusion.

(9.8.2) (a) $\rho_{k 1} / s_{k 1}=\max \left\{\rho_{k 1} / s_{k 1}, \rho_{k 2} / s_{k 2}, \ldots\right\}$ for every $k$; and

(b) Every $\rho_{k 1} / s_{k 1}$ is an integer.

Here $s_{k i}$ denotes the splitting number of the maximal ideal $M_{k i}^{\prime}$ of $\Gamma_{k}$ corresponding to the maximal ideal $M_{k i}$ of $\Omega_{k}$. We refer to these properties by saying that $(k, 1)$ is the $k$-indicator pair.

Claim 1. $s_{k 1}=1$ for every indicator pair $(k, 1)$. Choose $k$. Since maxspec $(\Omega)$ is $\Lambda$-connected, $M^{k 1}$ is $\Lambda$-linked to some $M^{h j} \neq M^{k 1}$. Hence, by Proposition 8.6, there is an indecomposable $(\bar{\Lambda}, \bar{\Omega})$-module of the form $\left[X \subset \bar{B}_{k 1} \oplus \bar{B}_{h j}\right]$. This yields the rank ratio $\rho_{k 1} / s_{k 1}=1 / s_{k 1}$, which must be an integer since $(k, 1)$ is an indicator pair. Hence $s_{k 1}=1$.

Claim 2. Some $\Omega_{k}$ has at least two maximal ideals. Suppose not. Since the sets of maximal ideals of $\Gamma$ and $\Omega$ are in one-to-one correspondence via contraction, the same is true of every $\Gamma_{k}$ and $\Omega_{k}$. Therefore, if every $\Omega_{k}$ were matrix-local, the same would be true of every $\Gamma_{k}$, and hence every pair $(k, i)$ would be the indicator pair $(k, 1)$. This, together with Claim 1 , yields the contradiction that we are in situation (i) of the statement of the theorem.

Now renumber the $\Omega_{k}$, if necessary, so that $\Omega_{1}$ has at least two maximal ideals $M_{11}, M_{12}$. 
Claim 3. $M^{12}$ is completely inertial over $\Lambda$. Otherwise, Proposition 8.7 yields an indecomposable $(\bar{\Lambda}, \bar{\Omega})$-module of the form $\left[X \subset \bar{B}_{12}\right]$ or $\left[X \subset \bar{B}_{12}^{2}\right]$. But then $\rho_{11} / s_{11}=0<\rho_{12} / s_{12}$, contradicting the maximum property of indicator pairs.

Claim 4. $M^{12}$ is $\Lambda$-linked to $M^{11}$ and to no other maximal ideal. As maxspec $(\Omega)$ is $\Lambda$-connected, $M^{12}$ must be linked to some $M^{h j}$. Proposition 8.6 then yields an indecomposable $(\bar{\Lambda}, \bar{\Omega})$-module of the form $\left[X \subset \bar{B}_{h j} \oplus \bar{B}_{12}\right]$. Since $\bar{B}_{12}$ occurs, the maximum property of indicator pairs requires $\bar{B}_{11}$ to occur also. Therefore $(h, j)=(1,1)$.

Claim 5. $s_{12}=1$. Each $\Omega_{k}$ is a full $n_{k} \times n_{k}$ matrix ring over an integral domain, by $[\mathrm{LO},(5.1 .2)]$, and the multiplicity $u_{k i}$ of $\bar{B}_{k i}$ as a direct summand of $\bar{\Omega}_{k}$ is $n_{k} s_{k i}$ by $[\mathrm{LO}, 6.19(\mathrm{ii})]$. Therefore Lemma 8.2 shows that $n_{1} s_{11} \geq n_{1} s_{12}$. Since $s_{11}=1$ this yields $s_{12}=1$, as desired. It also yields $u_{11}=u_{12}$.

Claim 6. $M^{11}$ is completely inertial over $\Lambda$. If not, then Proposition 8.11 yields an indecomposable $(\bar{\Lambda}, \bar{\Omega})$-module of the form $\left[Z \subset \bar{B}_{12}^{2} \oplus \bar{B}_{11}\right]$. For this module, we have

$$
\rho_{11} / s_{11}=1 / 1<2 / 1=\rho_{12} / s_{12},
$$

contradicting the maximum property of the indicator pair $(1,1)$.

Claim 7. $M^{11}$ is $\Lambda$-linked to no maximal ideal other than $M^{12}$. Suppose that $M^{11}$ is linked to $M^{h j}$. Then we have

$$
\left(M^{11} \cap \Lambda\right)+\left(M^{h j} \cap \Lambda\right) \subset \Lambda .
$$

Since $M^{11}$ is completely inertial over $\Lambda, M^{11} \cap \Lambda$ is a maximal ideal of $\Lambda$. Similarly, $M^{12} \cap \Lambda$ is a maximal ideal of $\Lambda$. Therefore the linkage of $M^{11}$ with $M^{12}$ shows that $M^{11} \cap \Lambda=M^{12} \cap \Lambda$; and so $M^{11}$ can be replaced by $M^{12}$ in (9.8.3). Unless $(h, j)=(1,2)$, statement $(9.8 .3)$ then contradicts the fact that $M^{12}$ is linked to no maximal ideal other than $M^{11}$ [Claim 4].

Thus $M^{11}$ and $M^{12}$ are linked to each other and to no other maximal ideals. Since $\operatorname{maxspec}(\Omega)$ is $\Lambda$-connected, this shows that $\operatorname{maxspec}(\Omega)=\left\{M^{11}, M^{12}\right\}$; thus it consists of precisely two maximal ideals, both are completely inertial over $\Lambda$, and $s_{11}=s_{12}=1$. Moreover $\Omega=\Omega_{1}$ (otherwise some $M^{k i}$ would have a subscript $k \neq 1$ ), and therefore each $M^{1 i}=M_{1 i}$.

Since every maximal ideal of every splitting order $\Omega$ is completely inertial over $\Lambda$, we conclude $\Gamma$ is module-finite over $R$ and $\Omega=\Gamma$ [Lemma 7.6].

Lemma 9.4 now yields a description of $\Lambda$ as an equalizer, and then Lemma 9.5 shows that $\Lambda=M_{n}\left(\Lambda^{\prime}\right)$ and $\Gamma=M_{n}\left(\Gamma^{\prime}\right)$ with $\Lambda^{\prime}$ and $\Gamma^{\prime}$ as in statement (ii)(a) of the theorem, provided that we verify that $\Gamma^{\prime} / M$ and $\Gamma^{\prime} / N$ are division rings. Since each of $M_{11}$ and $M_{12}$ has splitting number 1 , the same is true of the corresponding maximal ideals $M$ and $N$ of $\Gamma^{\prime}$. Since $\Gamma^{\prime}$ is an integral domain, this shows that $\Gamma^{\prime} / M$ and $\Gamma^{\prime} / N$ are division rings, as desired. This completes the proof of Case 1 .

Case 2: $\Gamma$ (hence $\Omega$ ) is matrix-local. We show that situation (ii)(b) holds. Since $\Gamma$ and $\Omega$ are matrix-local, we have $\Gamma=\Gamma_{1}$ and $\Omega=\Omega_{1}$. Let $M^{\prime}=M_{11}^{\prime}$, the maximal ideal of $\Gamma$. Then the maximal ideal of $\Omega$ is $M=M^{\prime} \cap \Omega$, also called $M_{11}$ in our general notation scheme. In Case 2 there is only one splitting number, $s=s_{11}$, and the array of ranks $\left(\left(\rho_{k i}\right)\right)$ of any $(\bar{\Lambda}, \bar{\Omega})$-module $[X \subseteq Y]$ reduces to the single number $\rho=\rho_{11}$. Moreover, every conductor ideal for $\Lambda$ and $\Omega$ is contained 
in $M=J(\Omega)$ and therefore in $J(\Lambda)$ [see (3.5.3)]. The part of the Fundamental Combinatorial Lemma quoted in (9.8.2) reduces to the much simpler statement:

(9.8.4) $\rho / s$ is an integer for every indecomposable $(\bar{\Lambda}, \bar{\Omega})$-module $[X \subset Y]$ with proper inclusion.

Moreover, $s \geq 2$ because we are not in situation (i) of the theorem.

Claim 1. If there exists a splitting order $\Omega^{\prime \prime}$ such that $\Omega^{\prime \prime} \subset \Omega$, then there is an indecomposable $(\bar{\Lambda}, \bar{\Omega})$-module of the form $[X \subset \bar{B}]$, where $\bar{B}=\bar{B}_{11}$ is the unique indecomposable projective left $\bar{\Omega}$-module.

By [LO, 6.19(ii)] we have $\bar{\Omega} \cong \bar{B}^{n s}$, as left $\bar{\Omega}$-modules, where $n$ is the integer such that $\Omega$ is an $n \times n$ matrix ring over an integral domain. Note that this multiplicity $n s$ is the same for all splitting orders $\Omega$.

Any conductor ideal $C$ for $\Lambda$ and $\Omega$ is also a conductor ideal for $\Lambda$ and $\Omega^{\prime \prime}$. We write $\bar{\Omega}^{\prime \prime}=\Omega^{\prime \prime} / C$, a matrix-local ring since the maximal ideals of any splitting order are the contractions of the maximal ideals of $\Gamma$. By the previous paragraph, we have $\bar{\Omega}^{\prime \prime} \cong\left(\bar{B}^{\prime \prime}\right)^{n s}$ for some $\bar{B}^{\prime \prime}$. Since the multiplicity $n s$ is the same for $\bar{\Omega}^{\prime \prime}$ as for $\bar{\Omega}$, and since $\bar{\Omega}^{\prime \prime} \subset \bar{\Omega}$ (proper inclusion), Claim 1 follows easily with $X=\bar{B}^{\prime \prime}$.

Claim 2. $\Gamma$ is module-finite over $R$. Suppose not. Then there exist two distinct splitting orders $\Omega^{\prime \prime} \subset \Omega$ containing $\Lambda$. To see this, let $\Omega^{\prime \prime}$ be any splitting order $\left(\Lambda \subseteq \Omega^{\prime \prime} \subseteq \Gamma\right)$. Since $\Gamma$ is not module-finite over $R$ there is an element $\gamma \in \Gamma-\Omega^{\prime \prime}$. Then, by [LO, 5.2], there is a splitting order $\Omega \subseteq \Gamma$ containing $\Omega^{\prime \prime}$ and $\gamma$. But then the indecomposable $(\bar{\Lambda}, \bar{\Omega})$-module $[X \subset \bar{B}]$ constructed in Claim 1 yields rank ratio $\rho / s=1 / s$, which is not an integer since $s \geq 2$; and this contradicts our combinatorial lemma (9.8.4), proving Claim 2.

Now that we know that $\Gamma$ is module-finite over $R$, and hence $\Gamma$ is a splitting order for $\Lambda$, we may take $\Gamma=\Omega$.

Claim 3. The maximal ideal $M$ of $\Omega$ is not completely inertial over $\Lambda$. For since $M=J(\Omega)$, we have $\bar{M}^{k}=0$ for some $k$, and hence $M^{k} \subseteq C \subseteq \Lambda$. But then $\Lambda+M^{k}=\Omega$ would imply $\Lambda=\Omega$, a contradiction.

Since $M$ is not completely inertial over $\Lambda$, Proposition 8.7 yields an indecomposable $(\bar{\Lambda}, \bar{\Omega})$-module of the form $[X \subset \bar{B}]$ or $\left[X \subset \bar{B}^{2}\right]$. The corresponding rank ratios $\rho_{11} / s$ are $1 / s$ and $2 / s$ respectively. Since this must be an integer and $s \geq 2$, we have $s=2$ as stated in the theorem.

Claim 4. Every $\Omega$-generator of $\bar{B}$ is a $\Lambda$-generator. Suppose not, and let $x$ be an $\Omega$-generator of $\bar{B}$ that is not a $\Lambda$-generator. Then the $(\bar{\Lambda}, \bar{\Omega})$-module $[\Lambda x \subset \Omega x]=$ $[\Lambda x \subset \bar{B}]$ is indecomposable and has rank ratio $1 / s=1 / 2 \notin \mathbb{Z}$, again contrary to our combinatorial lemma.

We now begin proving the assertions in statement (ii)(b) of the theorem. But we prove them about $\Lambda$ and $\Gamma$ rather than $\Lambda^{\prime}$ and $\Gamma^{\prime}$, because we do not prove that $\Lambda$ is an $n \times n$ matrix ring until the end of the proof. We already have that $\Gamma$ has exactly one maximal ideal and its splitting number is 2 .

The unique indecomposable projective $\bar{\Omega}$-module $\bar{B}$ has a unique maximal submodule $A$, and therefore the generator property in Claim 4 passes down to the unique simple $\bar{\Omega}$-module $\bar{B} / A \cong S$. It follows that every nonzero element of $S$ is a $\Lambda$-generator, and therefore $S$ remains simple as a $\Lambda$-module, as stated in the theorem.

Next we show that $\Gamma(M \cap \Lambda) \Gamma=M$. Since $\Omega=\Gamma$, a maximal order with local center, $\Omega$ is a principal ideal ring [LO, (4.1.8)]. Therefore $\bar{\Omega}$ is an artinian principal 
ideal ring; and hence every indecomposable $\bar{\Omega}$-module is uniserial [Ja ' 43 , Theorems $43,45]$. In particular, $\bar{B}$ is uniserial. Lemma 9.2 now yields the claimed property of $M$ when $\lambda$, the length of $\bar{B}$, is $\geq 2$.

Assume now that $\lambda=1$. Then the unique indecomposable projective left $\bar{\Omega}$ module $\bar{B}$ is simple. This shows that the artinian ring $\bar{\Omega}$ is a simple ring. It follows that the conductor ideal $C$ equals $M$, and therefore $M \subseteq \Lambda$. The desired relation $\Gamma(M \cap \Lambda) \Gamma=M$ is obvious, in this situation.

Since $\Gamma=\Gamma_{1}$ we have $\Gamma=M_{n}\left(\Gamma^{\prime}\right)$ for some PID $\Gamma^{\prime}[\mathrm{LO},(4.1 .8)]$. We now show that $\Lambda=M_{n}\left(\Lambda^{\prime}\right)$ for some $\Lambda^{\prime} \subset \Gamma^{\prime}$, as claimed in the theorem. It suffices to show:

(9.8.5) (i) Every primitive idempotent of $\Lambda$ remains primitive in $\Omega$; and (ii) All primitive idempotents of $\Lambda$ generate isomorphic left ideals of $\Lambda$.

For then, by (9.5.1), $\Lambda$ contains a set of $n \times n$ matrix units $\left\{d_{i j}\right\}$, and is therefore isomorphic to a full $n \times n$ matrix ring with entries in $d_{11} \Lambda d_{11}$. Moreover, these elements $d_{i j}$ form a set of $n \times n$ matrix units in the larger ring $\Gamma$, and therefore $\Gamma$ is isomorphic to a full $n \times n$ matrix ring with entries in the ring $d_{11} \Gamma d_{11}$ containing $d_{11} \Lambda d_{11}$. Thus it suffices to prove that $d_{11} \Gamma d_{11}$ is a PID (and hence can serve as a new $\Gamma^{\prime}$ ). This follows from the fact that $d_{11} \Gamma d_{11}$ is Morita-equivalent to $\Gamma$, hence to $\Gamma^{\prime}$.

Let $d$ be a primitive idempotent of $\Lambda$, and suppose that $d$ is not primitive in $\Omega$. Then $d$ is the sum of at least two orthogonal, primitive idempotents of $\Omega$. Since the splitting number $s$ equals 2, the image $\bar{d}$ of $d$ in $\bar{\Omega}$ is therefore the sum of at least four primitive idempotents of $\bar{\Omega}$. Say $\bar{d}=\sum_{i} \bar{e}_{i}$.

The $(\bar{\Lambda}, \bar{\Omega})$-module $L=[\bar{\Lambda} \bar{d} \subset \bar{\Omega} \bar{d}]$ cannot be indecomposable. For if it were, the $\bar{\Lambda}$-module $\bar{\Lambda} \bar{d}$ would be indecomposable [Lemma 8.4], and therefore the $(\bar{\Lambda}, \bar{\Omega})$ module $L^{\prime}=\left[\pi(\bar{\Lambda} \bar{d}) \subseteq \bigoplus_{i=1}^{3} \bar{\Omega}_{\bar{e}}\right]$ (where $\pi$ is the projection map to $\bigoplus_{i=1}^{3} \bar{\Omega} \bar{e}_{i}$ ) would also be indecomposable [Lemma 8.5]. Note that indecomposability forces the inclusion in $L^{\prime}$ to be strict. But the rank ratio associated with $L^{\prime}$ is $\rho / s=3 / 2$, contradicting the combinatorial lemma, which requires this ratio to be an integer.

At this point it is important to distinguish between indecomposable modules and indecomposable packages. Call a $(\bar{\Lambda}, \bar{\Omega})$-module $[X \subseteq Y]$ a package if there is a torsionfree $\Lambda$-module $A$ such that $\Omega A$ is $\Omega$-projective and $[X \subseteq Y] \cong[A / C A \subseteq$ $\Omega A / C A]$; and call a package indecomposable if it is not the direct sum of two nonzero packages, and $X \neq 0$. Since $\Lambda d$ is an indecomposable projective $\Lambda$-module, the $(\bar{\Lambda}, \bar{\Omega})$-module $L$ at the beginning of the previous paragraph is an indecomposable package; but it is not an indecomposable $(\bar{\Lambda}, \bar{\Omega})$-module, as we have shown.

Since $s=2$, the Package Deal Theorem for $(\bar{\Lambda}, \bar{\Omega})$-modules [LO, 6.21] shows that a $(\bar{\Lambda}, \bar{\Omega})$-module $[X \subseteq Y]$ is a package if and only if its rank $\rho=\rho_{11}$, is an even number. Now consider any proper decomposition $L=V \oplus W$ of the decomposable $(\bar{\Lambda}, \bar{\Omega})$-module $L$. Since $L$ is a package its rank is even. The rank of $V$ cannot be even, because then the rank of $W$ would also be even, contradicting indecomposability of $L$ as a package. Therefore $V$ has odd rank. But then the rank ratio $\rho(V) / s=\rho_{11} / 2$ is not an integer, contradicting the combinatorial lemma. This contradiction completes the proof of (9.8.5)(i).

Before proving (9.8.5)(ii) we first prove

Claim $\alpha$. All primitive idempotents of $\bar{\Lambda}$ generate isomorphic left ideals of $\bar{\Lambda}$. By liftability of idempotents modulo the radical in artinian rings, it suffices to prove this for $\bar{\Lambda}$ modulo its radical. Since $\Lambda$ is matrix-local, so is $\bar{\Lambda}$; and so $\bar{\Lambda}$ becomes simple modulo its radical. The truth of Claim $\alpha$ is now clear. 
Claim $\beta$. If some primitive idempotent $\bar{d}$ of $\bar{\Lambda}$ becomes the sum of (say) $n$ orthogonal idempotents in $\bar{\Omega}$, then the same is true of all primitive idempotents of $\bar{\Lambda}$. This holds since all primitive idempotents $\bar{d}$ of $\bar{\Lambda}$ generate isomorphic left ideals $\bar{\Lambda} \bar{d}$ of $\bar{\Lambda}$, and since $\bar{\Omega} \bar{d} \cong \bar{\Omega} \otimes_{\bar{\Lambda}} \bar{\Lambda} \bar{d}$.

The following commutative diagram displays a primitive idempotent $d \in \Lambda$, and the facts that it remains primitive in $\Omega$ and then splits into the sum of two orthogonal primitive idempotents in $\bar{\Omega}$. We examine what happens in $\bar{\Lambda}$.

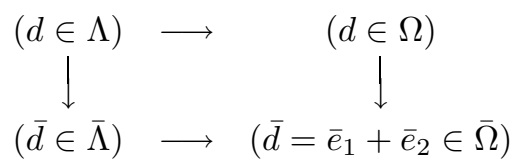

Commutativity of the diagram (and KS for $\bar{\Omega}$-modules) shows that $\bar{d}$ either is primitive in $\bar{\Lambda}$ or else is the sum of two primitive idempotents in $\bar{\Lambda}$. We consider these situations separately.

(a) $\bar{d}$ is a sum of two orthogonal idempotents in $\bar{\Lambda}$. Say $\bar{d}=\bar{c}_{1}+\bar{c}_{2}$. Then $\bar{c}_{1}$ remains primitive in $\bar{\Omega}$ (otherwise $d$ splits into the direct sum of more than two orthogonal idempotents in $\bar{\Omega}$ ). Therefore, by Claim $\beta$, every primitive idempotent of $\bar{\Lambda}$, remains primitive in $\bar{\Omega}$.

(b) $\bar{d}$ is primitive in $\bar{\Lambda}$. Then $\bar{d}$, hence every primitive idempotent of $\bar{\Lambda}$, splits into a sum of two orthogonal primitive idempotents in $\bar{\Omega}$.

Since the situations described in the preceding two paragraphs are mutually exclusive, we conclude that either situation (a) holds for all $d \in \Lambda$ or else situation (b) holds for all $d$. In either case Claim $\alpha$ shows that $\bar{\Lambda} \bar{d} \cong \bar{\Lambda} \bar{d}^{\prime}$ for all primitive idempotents $d, d^{\prime}$ in $\Lambda$. Since such isomorphisms lift modulo ideals contained in the radical [by uniqueness of the projective cover, 8.8] we get $\Lambda d \cong \Lambda d^{\prime}$; and this concludes the proof of (9.8.5)(ii), hence of Case 2.

Proof 9.9 (of Supplement 9.7). (a). The statement about $J\left(\Lambda^{\prime}\right)$ is part of Lemma 9.5. Hence $\Lambda^{\prime} / J\left(\Lambda^{\prime}\right)=\Lambda^{\prime} /\left(M \cap \Lambda^{\prime}\right)$, which, by the definition of "equalizer", is isomorphic to $\Gamma^{\prime} / M$, a division ring by hypothesis. Hence $\Lambda^{\prime}$ is local. Since $\Lambda^{\prime}$ is integral over $Z\left(\Lambda^{\prime}\right), M \cap Z\left(\Lambda^{\prime}\right)$ is a maximal ideal of $Z\left(\Lambda^{\prime}\right)$. If $Z\left(\Lambda^{\prime}\right)$ had another maximal ideal, the inclusion $Z\left(\Lambda^{\prime}\right) \subset \Lambda^{\prime}$ (together with the fact that $\Lambda^{\prime}$ is local) would yield a contradiction of the lying-over theorem for finite centralizing extensions of noetherian rings [MR '87, 10.2.9].

(b). This is more complicated than the proof of (a). Since we have no need for the original $\Lambda$ and $\Gamma$, we can simplify the notation by writing $\Lambda$ and $\Gamma$ in place of $\Lambda^{\prime}$ and $\Gamma^{\prime}$ respectively. As in our standard notation, let $C$ denote a conductor ideal for $\Lambda$ and $\Gamma$, and note that $C$ is necessarily contained in the unique maximal ideal $M=J(\Gamma)$. Therefore, by (3.5.3), $C \subseteq J(\Lambda)$.

The ideal $J(\Gamma)$ of $\Gamma$ is the annihilator in $\Gamma$ of the unique simple $\Gamma$-module $S$; and therefore the annihilator of $S$ in $\Lambda$ is $J(\Gamma) \cap \Lambda$. But then the artinian ring $\Lambda /(J(\Gamma) \cap \Lambda)$ has $S$ as a faithful simple module, and is therefore a simple ring. In other words, $J(\Gamma) \cap \Lambda=M \cap \Lambda$ is a maximal ideal of $\Lambda$, and hence $J(\Gamma) \cap \Lambda \supseteq J(\Lambda)$. The opposite inclusion follows easily by reducing modulo $C$, because the radical $J(\Gamma) / C$ of the artinian ring $\Gamma / C$ is nilpotent. Thus $J(\Lambda)=M \cap \Lambda$ as desired.

We still need to show that $\Lambda$ and $Z(\Lambda)$ are local rings. Consider $\Lambda$ first. Since $J(\Lambda)$ is a maximal ideal, $\Lambda$ is matrix-local. As usual, let $\bar{\Lambda}=\Lambda / C$ and $\bar{\Gamma}=\Gamma / C$. Since $C \subseteq J(\Lambda)$ it suffices to show that $\bar{\Lambda}$ is local; and since $\bar{\Lambda}$ is artinian, it suffices 
to show that $\bar{\Lambda}$ has no nontrivial idempotents. So let $1=\sum_{i=1}^{s} d_{i}$ where the $d_{i}$ are primitive orthogonal idempotents of $\bar{\Lambda}$. We want to prove that $s=1$. We have

$$
\bar{\Gamma}=\bigoplus_{i} \bar{\Gamma} d_{i}
$$

Since $M=J(\Gamma)$ has splitting number 2 , we have $s \leq 2$, so we may assume (in order to find a contradiction) $s=2$ and the $d_{i}$ remain primitive in $\bar{\Gamma}$. Since $\Gamma$ is PID $[\mathrm{LO},(4.1 .8)], \bar{\Gamma}$ is an artinian principal ideal ring, and hence every indecomposable projective $\bar{\Gamma}$-module is uniserial [Ja ' 43 , Theorems 43,45]. Therefore, by hypothesis (ii)(b) of Theorem 9.6, the generator property in Lemma 9.2 shows that each $\bar{\Gamma} d_{i}=$ $\bar{\Lambda} d_{i}$. But then (9.9.1) implies that $\bar{\Gamma}=\bar{\Lambda}$, contradicting the hypothesis $\Gamma \neq \Lambda$. Thus $\bar{\Lambda}$ is a local ring, and hence so is $\bar{\Lambda}$.

As at the end of the proof of statement (a), this proves that $Z(\Lambda)$ is also local.

\section{Sufficient Conditions for Local TFKS}

In this section we prove that the rings $\Lambda$ described in statement (ii) of Local Necessity Theorem 9.6 actually satisfy TFKS.

Notation 10.1. Throughout this section $R=Z(\Lambda)$ is local, the normalization $\Gamma$ of $\Lambda$ is module finite over $R$, and $\Gamma \neq \Lambda$. In this situation $\Gamma$ becomes a splitting order, in fact, a maximal order. Consequently all of our standard notation that applies to an arbitrary splitting order $\Omega$ can now be applied to $\Gamma$. In particular, there exist conductor ideals $C$ for $\Lambda$ and $\Gamma$, and $\Lambda$ is the pullback of its conductor square with respect to $C$, as displayed in (3.5.4).

To prove our sufficiency theorem we convert the description of the structure of an arbitrary $(\bar{\Lambda}, \bar{\Gamma})$-module to a matrix problem, and then solve enough of this problem to prove the theorem. We now describe our notation for the matrix problem, using our version of a procedure of Green and Reiner [GR '78].

We define a $(\bar{\Lambda}, \bar{\Gamma})$-map $f: W \rightarrow Y$ to be a $\bar{\Lambda}$-homomorphism, where

(10.1.1) $W$ is a projective $\bar{\Lambda}$-module, $Y$ is a projective $\bar{\Gamma}$-module, $\operatorname{ker}(f) \subseteq J(\bar{\Lambda}) \cdot W$, and $\bar{\Gamma}(W f)=Y$.

We will consistently write such maps on the right, in this discussion. Note that every such $f$ yields a natural $(\bar{\Lambda}, \bar{\Gamma})$-module, namely $[W f \subseteq Y]$. We call this the $(\bar{\Lambda}, \bar{\Gamma})$-module represented by $f$. Conversely, let $[X \subseteq Y]$ be a $(\bar{\Lambda}, \bar{\Gamma})$-module, and let $f: W \rightarrow X$ be a $\bar{\Lambda}$-projective cover of $X$ [Remarks 8.8]. Then we can view $f$ as a $(\bar{\Lambda}, \bar{\Gamma})$-map $W \rightarrow Y$.

We call $(\bar{\Lambda}, \bar{\Gamma})$-maps $f, g: W \rightarrow Y$ equivalent, and write $f \sim g$, if $g=\phi f \theta$ for automorphisms $\phi \in \operatorname{Aut}_{\bar{\Lambda}}(W)$ and $\theta \in \operatorname{Aut}_{\bar{\Gamma}}(Y)$. Since the map $W \rightarrow W f$ is a projective cover, it follows from uniqueness of the projective cover [8.8] that

$$
f \sim g \Longleftrightarrow[W f \subseteq Y] \cong[W g \subseteq Y] .
$$

If $W=\bigoplus_{i} W_{i}$ and $Y=\bigoplus_{j} Y_{j}$ are $\bar{\Lambda}$ and $\bar{\Gamma}$-module decompositions of $W$ and $Y$ respectively, then we can regard any $(\bar{\Lambda}, \bar{\Gamma})$-map $f: W \rightarrow Y$ as a "matrix" $\left(\left(f_{i j}\right)\right)$ where each $f_{i j}$ is a $\bar{\Lambda}$-homomorphism $W_{i} \rightarrow Y_{j}$.

By uniqueness of projective covers, the $(\bar{\Lambda}, \bar{\Gamma})$-module represented by a nonzero $f$ is indecomposable if and only if there are no decompositions $W=W_{1} \oplus W_{2}$ and $Y=Y_{1} \oplus Y_{2}$ with respect to which the matrix of $f$ becomes a $2 \times 2$ diagonal matrix with both $f_{11} \neq 0$ and $f_{22} \neq 0$. 
We now consider the situations in (ii)(a) and (ii)(b) of the Local Necesssity Theorem separately, beginning with the easier one. In both situations, it suffices, by Morita equivalence, to show that $\Lambda^{\prime}$ satisfies TFKS. Therefore we may simplify the notation by supposing that $\Lambda=\Lambda^{\prime}$ and $\Gamma=\Gamma^{\prime}$. In particular, $\Lambda$ and $\Gamma$ are integral domains, and $\Gamma$ is a PID.

10.2. Situation (ii)(a). In this subsection we suppose that

(10.2.1) $\Gamma$ has exactly two maximal ideals $M, N$, both of them have splitting number 1 , and $\Lambda$ is an equalizer $\Lambda=\mathrm{eq}(\Gamma, f, g)$ for some surjective $f, g$ and some $h \geq 1$ such that $\operatorname{ker}(f)=M^{h}$ and $\operatorname{ker}(g)=N^{h}$.

Let $\bar{\Lambda}=f(\Gamma)=g(\Gamma)$. Since the ideals $M^{h}$ and $N^{h}$ are comaximal, the map $[f, g]: \Gamma \rightarrow \bar{\Lambda} \oplus \bar{\Lambda}$ is a surjection, and therefore $f$ and $g$ map the equalizer $\Lambda$ onto $\bar{\Lambda}$. Setting $C=\operatorname{ker}(f) \cap \operatorname{ker}(g)$ we have $\Gamma / C \cong \bar{\Lambda} \oplus \bar{\Lambda}$. With this notation, $\Lambda$ becomes the pullback of the conductor square displayed in (9.4.1), and the bottom line of this square is the diagonal imbedding

$$
\bar{\Lambda} \rightarrow \bar{\Gamma}=\bar{\Lambda} \oplus \bar{\Lambda} .
$$

The ideal $C=\operatorname{ker}(f) \cap \operatorname{ker}(g)$ is the maximal conductor ideal for $\Lambda$ and $\Gamma$, by the definition of "equalizer", and $J(\Gamma) \supseteq C$. We also have $J(\Lambda)=M \cap \Lambda=N \cap \Lambda$ by Lemma 9.5.

$\bar{\Lambda}$ is a local ring, by Supplement 9.7. In particular, $\bar{\Lambda}$ has no nontrivial idempotents and (as in the commutative case) all projective $\bar{\Lambda}$-modules are free. Since $\Gamma$ is a maximal $R$-order in a division ring and $R$ is semilocal, $\Gamma$ is a (left and right) principal ideal domain $[\mathrm{LO},(4.1 .8)]$. Therefore, for every matrix $f$ over the homomorphic image $\bar{\Lambda}$ of $\Gamma$, there exist invertible matrices $a, b$ over $\bar{\Lambda}$ such that $a$ f $b$ is a diagonal matrix [Ja '43, Theorem 16].

The indecomposable projective left $\bar{\Gamma}$-modules are $\bar{B}_{M}=(\bar{\Lambda}, 0)$ and $\bar{B}_{N}=(0, \bar{\Lambda})$. The crux of our proof is the following fact:

(10.2.3) Every indecomposable $(\bar{\Lambda}, \bar{\Gamma})$-module not isomorphic to $\left[\bar{B}_{M} \subseteq \bar{B}_{M}\right]$ or $\left[\bar{B}_{N} \subseteq \bar{B}_{N}\right]$ is of the form $\left[X \subset \bar{B}_{M} \oplus \bar{B}_{N}\right]$.

To prove this, let $f: W \rightarrow Y$ be any $(\bar{\Lambda}, \bar{\Gamma})$-map that represents an indecomposable $(\bar{\Lambda}, \bar{\Gamma})$-module. Since $W$ and $Y$ are projective modules over $\bar{\Lambda}$ and $\bar{\Gamma}$ respectively, we can write $W=\bar{\Lambda}^{k}$ and $Y=\bar{B}_{M}^{u} \oplus \bar{B}_{N}^{v}$ for suitable integers $k, u, v$. Since every left $\bar{\Lambda}$-endomorphism of $\bar{\Lambda}$ equals right multiplication by a unique element of $\bar{\Lambda}$, the map $f$ equals right multiplication by a $k \times(u+v)$ matrix of elements of $\bar{\Lambda}$. It is convenient to partition this matrix $f$ into a pair of matrices $f=\left[f_{M} \mid f_{N}\right]$ where $f_{M}$ has size $k \times u$ and $f_{N}$ has size $k \times v$.

By the discussion preceding (10.1.2) an arbitrary $(\bar{\Lambda}, \bar{\Gamma})$-map equivalent to $f$ can be obtained from $f$ by means of the following matrix operations: (i) simultaneously left multiply $f_{M}$ and $f_{N}$ by an invertible matrix over $\bar{\Lambda}$; and (ii) (independently) right-multiply $f_{M}$ and $f_{N}$ by invertible matrices over $\bar{\Lambda}$ (since $\bar{\Gamma}=\bar{\Lambda} \oplus \bar{\Lambda}$ ). We now simplify the form of $f$ by applying these operations.

Choose invertible matrices $a, b$ such that $a f_{M} b$ is a diagonal matrix. Since $\bar{\Gamma}(W f)=Y=\bar{\Lambda}^{u} \oplus \bar{\Lambda}^{v}$, and $\bar{\Gamma}=\bar{\Lambda} \oplus \bar{\Lambda}$ (ring-direct sum, where the first $\bar{\Lambda}$ acts on $\bar{\Lambda}^{u}$ and the second $\bar{\Lambda}$ acts on $\bar{\Lambda}^{v}$ ), the rows of $f_{M}$ generate $\bar{\Lambda}^{u}$ as a left $\bar{\Lambda}$-module. Therefore the same is true of the diagonal matrix $a f b$; and its diagonal entries are therefore units of $\bar{\Lambda}$. After right-multiplying $b$ by an appropriate diagonal matrix 
with units on its diagonal we therefore have

$$
\left[f_{M} \mid f_{N}\right] \sim\left[\begin{array}{c|c}
I_{u} & f_{1} \\
0 & f_{2}
\end{array}\right]
$$

where the column consisting of $f_{1}$ and $f_{2}$ equals $a f_{N}$.

Now choose invertible matrices $a, b$ such that $a f_{2} b$ is a diagonal matrix. Do this in such a way that all units occurring on the main diagonal equal 1 and occur before any nonunits occur. This yields

$$
\left[f_{M} \mid f_{N}\right] \sim\left[\begin{array}{c|cc}
I_{u} & f_{3}^{\prime} & f_{3} \\
0 & I_{r} & 0 \\
0 & 0 & f_{4}
\end{array}\right]
$$

where $\left[f_{3}^{\prime} f_{3}\right]=f_{1} b$ and the diagonal matrix $f_{4}$ has its entries in $J(\bar{\Lambda})$. By adding suitable multiples of the rows containing $I_{r}$ to the rows above it, transform $f_{3}^{\prime}$ to zero. (This can be accomplished by simultaneously left-multiplying both halves of the matrix by an appropriate invertible matrix.)

Note that if $r \geq 1$ (that is, if $I_{r}$ actually occurs) we now have a direct summand of the $(\bar{\Lambda}, \bar{\Gamma})$-module determined by $f$. This summand is isomorphic to $\left[\bar{B}_{N} \subseteq \bar{B}_{N}\right]^{r}$. Therefore, by our indecomposability assumption, the $(\bar{\Lambda}, \bar{\Gamma})$-module represented by $f$ is $\left[\bar{B}_{N} \subseteq \bar{B}_{N}\right]$, one of the indecomposables listed in statement (10.2.3). We can therefore suppose, from now on, that $r=0$. In other words:

$$
\left[f_{M} \mid f_{N}\right] \sim\left[\begin{array}{c|c}
I_{u} & f_{3} \\
0 & f_{4}
\end{array}\right] .
$$

Now choose invertible matrices $a, b$ such that $a f_{3} b$ is a diagonal matrix, where all units appearing on the main diagonal equal 1 . In order to replace $f_{3}$ by $a f_{3} b$ we must also replace $f_{4}$ by $f_{4} b$ and $I_{u}$ by $a I_{u}$. However, $a I_{u}$ can be transformed back to $I_{u}$ by right-multiplying the first $u$ columns by $a^{-1}$.

Since the rows of $f_{N}$ generate $\bar{\Lambda}^{v}$, and the entries of $f_{4}$ (and hence of $f_{4} b$ ) lie in $J(\bar{\Lambda})$, we see that the rows of the diagonal matrix $a f_{3} b$ generate $\bar{\Lambda}^{v}$. Therefore $a f_{3} b$ consists of $I_{v}$ followed by rows of zeros. We now have:

$$
\left[f_{M} \mid f_{N}\right] \sim\left[\begin{array}{cc|c}
I_{v} & 0 & I_{v} \\
0 & I_{u-v} & 0 \\
0 & 0 & f_{4} b
\end{array}\right] .
$$

If $u-v \neq 0$, then the indecomposable $(\bar{\Lambda}, \bar{\Gamma})$-module determined by $f$ has $\left[\bar{B}_{M} \subseteq\right.$ $\left.\bar{B}_{M}\right]$ as a direct summand and is therefore isomorphic to $\left[\bar{B}_{M} \subseteq \bar{B}_{M}\right]$, one of the indecomposable modules described in (10.2.3). Therefore we can assume, for the rest of the proof, that $u=v$; that is, the blocks of rows and columns containing $I_{u-v}$ do not actually occur.

Next we delete the factor $b$ by right-multiplying both sides of the matrix by $b^{-1}$ and then left-multiplying the rows containing $I_{v}$ (in both halves of the matrix) by $b$.

Since all entries of the diagonal matrix $f_{4}$ lie in $J(\bar{\Lambda})$, the matrix $f$ is now a direct sum of matrices of the form

$$
\left[\begin{array}{l|l}
1 & 1 \\
0 & j
\end{array}\right]
$$


with $j \in J(\bar{\Lambda})$. By indecomposability, $f$ is therefore a single matrix of this form, and therefore yields a $(\bar{\Lambda}, \bar{\Gamma})$-module of the form $\left[X \subset \bar{B}_{M} \oplus \bar{B}_{N}\right]$. (The inclusion must be strict because of indecomposability.) This completes the proof of statement (10.2.3).

Finally, we show that TFKS holds for $\Lambda$ by applying our Fundamental Combinatorial Lemma 4.3. Since $\Gamma$ is a maximal $R$-order, the class of torsionfree $\Lambda$ modules $L$ such that $\Gamma L$ is $\Gamma$-projective coincides with the class of all torsionfree $\Lambda$-modules. In statement (10.2.3) we showed that, for all indecomposable $(\bar{\Lambda}, \bar{\Gamma})$ modules $[X \subset Y]$ in which the inclusion is strict, the array of ranks of $[X \subset Y]$ is the single row $\rho=(1,1)$. Since both $M=M_{11}$ and $N=M_{12}$ have splitting number 1 , this array of ranks is also the array of rank ratios. Therefore both the maximum and integrality properties required by the combinatorial lemma are satisfied in a trivial way; and this shows that TFKS holds for $\Lambda$.

10.3. Situation (ii)(b). Recall that, as mentioned just before subsection 10.2, we may assume that $\Lambda$ and $\Gamma$ are integral domains, with $\Gamma$ a PID. As usual, fix a conductor ideal $C \subseteq J(\Lambda) \cap J(\Gamma)$ and set $\bar{\Lambda}=\Lambda / C$ and $\bar{\Gamma}=\Gamma / C$. Throughout the present subsection we assume, in adddition to the notation in 10.1, that:

(10.3.1) $\Gamma$ is matrix-local and its maximal ideal $J(\Gamma)=M=M_{11}$ has splitting number $s=s_{11}=2$. Moreover, the unique simple left $\Gamma$-module $S$ remains simple as a $\Lambda$-module, $\Lambda$ is a local domain with maximal ideal $J(\Lambda)=M \cap \Lambda$, and $\Gamma(M \cap \Lambda) \Gamma=M$.

We claim: These properties of $\Lambda$ are left-right symmetric (i.e. the simple right $\Gamma$-module remains $\Lambda$-simple). To prove this, let $E=\Gamma / J(\Gamma)$, a simple artinian ring since $\Gamma$ is matrix-local, and let $D$ be the natural image of $\Lambda$ in $E$. Then apply Lemma 10.4, below.

Before considering TFKS we discuss some details of the structure of $\bar{\Lambda}$ and $\bar{\Gamma}$. Since $\Gamma$ is a matrix-local PID, $\bar{\Gamma}$ is a matrix-local artinian principal ideal ring. It follows that $\bar{\Gamma}$ is a full matrix ring over an artinian valuation ring $A$, that is, over an artinian ring $A$ whose one-sided ideals are all 2 -sided and are totally ordered by inclusion [Ja '43, Theorems 37, 38, 39]. (Alternatively, pass to the completion and use [R '75, Theorem 17.3].) Since $s=2$ and $\Gamma$ is an integral domain, we have $\bar{\Gamma}=M_{2}(A)$.

The unique indecomposable projective left $\bar{\Gamma}$-module $\bar{B}$ is uniserial, since $A$ is a valuation ring. Therefore, Lemma 9.2 establishes the following "generator property" of $\bar{B}$ : Every $\bar{\Gamma}$-generator of $\bar{B}$ is a $\bar{\Lambda}$-generator. Since $\bar{B} \cong \bar{\Gamma}\left[\begin{array}{l}1 \\ 0\end{array}\right]$ we have:

$$
\bar{\Lambda}\left[\begin{array}{l}
1 \\
0
\end{array}\right]=\bar{\Gamma}\left[\begin{array}{l}
1 \\
0
\end{array}\right] \text {. }
$$

The local subring ring $\bar{\Lambda}$ of $\bar{\Gamma}=M_{2}(A)$ has no nontrivial idempotents. On the other hand, we claim that $\bar{\Lambda}$ is a "large part" of $\Gamma$ in the following sense. For every pair of elements $x, y \in A, \bar{\Lambda}$ contains matrices of the following four forms:

$$
\left[\begin{array}{ll}
x & y \\
* & *
\end{array}\right], \quad\left[\begin{array}{ll}
* & * \\
x & y
\end{array}\right], \quad\left[\begin{array}{ll}
x & * \\
y & *
\end{array}\right], \quad\left[\begin{array}{ll}
* & x \\
* & y
\end{array}\right] .
$$

The matrices of the third form displayed in (10.3.3) occur in $\Lambda$ because of (10.3.2). The fourth form is similarly obtained. The matrices of the first two forms occur because, as proved above, our hypotheses on $\Lambda$ and $\Gamma$ are left-right symmetric. In 
other words, start with the indecomposable projective right $\bar{\Gamma}$-module $\left[\begin{array}{ll}1 & 0\end{array}\right] \bar{\Gamma}$ and repeat the proof given at the beginning of this paragraph.

Next we claim that, if $x, y \in A$ and at least one of them is a unit, then there is a unit $U \in \bar{\Lambda}$ such that

$$
U\left[\begin{array}{l}
x \\
y
\end{array}\right]=\left[\begin{array}{l}
1 \\
0
\end{array}\right]
$$

Since $x$ or $y$ is a unit, the matrix $\left[\begin{array}{l}x \\ y\end{array}\right]$ is a $\bar{\Gamma}$-generator of $\bar{\Gamma}\left[\begin{array}{l}1 \\ 0\end{array}\right]$, hence a $\bar{\Lambda}$-generator. Therefore there is some element $U \in \bar{\Lambda}$ such that (10.3.4) holds. Similarly there is an element $V \in \bar{\Lambda}$ such that $V\left[\begin{array}{l}1 \\ 0\end{array}\right]=\left[\begin{array}{l}x \\ y\end{array}\right]$. We still need to show that $U$ is a unit. By (10.3.1) $\bar{\Lambda}$ is a local ring with maximal ideal $J(\bar{\Gamma}) \cap \bar{\Lambda}$, so if $U$ is a non-unit we must have $U V \in J(\bar{\Gamma})$. Then $\bar{\Gamma}\left[\begin{array}{l}1 \\ 0\end{array}\right]=\bar{\Gamma} U V\left[\begin{array}{l}1 \\ 0\end{array}\right] \subseteq J(\bar{\Gamma})\left[\begin{array}{l}1 \\ 0\end{array}\right]$, a contradiction, and the claim is established.

We prove TFKS in situation (ii)(b) by invoking Fundamental Combinatorial Lemma 4.3, with $\Omega=\Gamma$ and $C$ any conductor ideal contained in $J(\Gamma) \bigcap J(\Lambda)$. Since $\Gamma$ is matrix-local, the array of ranks $\left(\left(\rho_{k i}\right)\right)$ of an arbitrary $(\bar{\Lambda}, \bar{\Gamma})$-module $[X \subseteq Y]$ becomes the single number $\rho=\rho_{11}$; and therefore $Y \cong \bar{B}^{\rho}$. What must be proved therefore takes a very simple form: For every indecomposable $(\bar{\Lambda}, \bar{\Gamma})$ module $\left[X \subset \bar{B}^{\rho}\right]$ with strict inclusion, the rank ratio $\rho / s=\rho / 2$ is an integer. It therefore suffices to show: Every $(\bar{\Lambda}, \bar{\Gamma})$-module of the form $L=\left[X \subset \bar{B}^{2 r+1}\right]$ is decomposable.

We claim that $r>0$. For otherwise, by the definition of a $(\bar{\Lambda}, \bar{\Gamma})$-module, we have $\bar{\Gamma} X=\bar{B}$. Since $\bar{B}$ is a uniserial $\Gamma$-module, $X$ therefore contains a $\bar{\Gamma}$-generator of $\bar{B}$. Our "generator condition" then implies that $X=\bar{B}$; and this contradiction of strictness of the inclusion $X \subset \bar{B}$ proves the claim.

Let $f: W \rightarrow \bar{B}^{2 r+1}$ be a $(\bar{\Lambda}, \bar{\Gamma})$-map that represents $\left[X \subset \bar{B}^{2 r+1}\right]$. Since the artinian ring $\bar{\Lambda}$ has no nontrivial idempotents, the projective $\bar{\Lambda}$-module $W$ is free. Say $W=\bar{\Lambda}^{m}$. Thus $f$, viewed as a matrix, is an element of $\operatorname{Hom}_{\bar{\Lambda}}(\bar{\Lambda}, \bar{B})_{m \times(2 r+1)}$, the set of $m \times(2 r+1)$ matrices with entries in $\operatorname{Hom}_{\bar{\Lambda}}(\bar{\Lambda}, \bar{B})$. We will show that

$$
f \sim \operatorname{diag}(g, h) \quad \text { with } g \in \operatorname{Hom}_{\bar{\Lambda}}(\bar{\Lambda}, \bar{B})_{(m-1) \times 2 r} \text { and } h \in \operatorname{Hom}_{\bar{\Lambda}}(\bar{\Lambda}, \bar{B}) .
$$

We can take $\bar{B}=A_{2 \times 1}$. Every $\theta \in \operatorname{Hom}_{\bar{\Lambda}}(\bar{\Lambda}, \bar{B})$ equals right multiplication by some unique element $\mu \in \bar{B}$; namely by $\mu=\theta(1)$. Therefore we have

$$
f \in \operatorname{Hom}_{\bar{\Lambda}}(\bar{\Lambda}, \bar{B})_{m \times(2 r+1)}=A_{2 m \times(2 r+1)}
$$

where the equal sign identifies $f_{i j} \in \operatorname{Hom}_{\bar{\Lambda}}(\bar{\Lambda}, \bar{B})$ with $f_{i j}(1) \in A_{2 \times 1}$.

The general form of a matrix equivalent to $f$ is $U f V$ where $U, V$ are automorphisms of the left $\bar{\Lambda}$-modules $\bar{\Lambda}^{m}, \bar{B}^{2 r+1}$ respectively. It is crucial, in the matrix reduction that follows, to understand the action of these automorphisms on $f$.

The automorphism $U$ is a square matrix of elements of $\bar{\Lambda}$. Each entry of $U$ is an element of $\bar{\Lambda} \subseteq A_{2 \times 2}$ and acts on the appropriate entries $f_{i j} \in A_{2 \times 1}$ by ordinary matrix multiplication. Similarly, each product $f_{i j} v$, where $v$ is an entry of $V$, is the ordinary matrix product $f_{i j} v \in A_{2 \times 1} A=A_{2 \times 1}$. We view $f$ as an element of $A_{2 m \times(2 r+1)}$, in this way, for the rest of the matrix reduction.

Note that the equivalence class of $f$ is unchanged by arbitrary elementary column operations, as well as by arbitrary elementary row-pair operations; that is:

(10.3.7) (a) Interchange some row-pair with some other row-pair;

(b) Left multiply a row-pair by a unit of $\bar{\Lambda}$;

(c) Add a left $\bar{\Lambda}$-multiple of some row-pair to a different row-pair. 
Note that whenever we refer to a row-pair, say rows $i$ and $i+1$, the number $i$ is always odd.

We have $\bar{\Gamma}(W f)=\bar{B}^{2 r+1}$, by (10.1.1); that is, the row-pairs of $f$ generate $\bar{B}^{2 r+1}=A_{2 \times(2 r+1)}$ as a left $\bar{\Gamma}$-module. Since $A$ is a local ring, this implies that $f$ has a unit in every column. A row-pair interchange therefore allows us to put a unit into one of the first two entries of column 1. The matrix is now of the form

$$
\left[\begin{array}{ll}
x & * \\
y & * \\
* & *
\end{array}\right] .
$$

After left-multiplying the first row-pair by the unit $U$ in (10.3.4) we get $\left[\begin{array}{l}x \\ y\end{array}\right]=\left[\begin{array}{l}1 \\ 0\end{array}\right]$.

By the "generator condition" we have $\bar{\Lambda}\left[\begin{array}{l}1 \\ 0\end{array}\right]=A_{2 \times 1}$. Therefore we can row-sweep the first column; that is, add appropriate left $\bar{\Lambda}$-multiples of the first row-pair to other row-pairs so that all entries in the first column below the first entry become zero. We can also column-sweep the first row. Our matrix now takes the form:

$$
f \sim\left[\begin{array}{ccccc}
1 & 0 & 0 & \cdots & 0 \\
0 & z & * & \cdots & * \\
0 & & & & \\
\vdots & & & f_{1} & \\
0 & & & &
\end{array}\right]
$$

for some $z \in A$ and $f_{1} \in A_{(2 m-2) \times 2 r}$. If $z$ is a unit we can right-multiply column 2 by $z^{-1}$ and obtain $z=1$.

Suppose that $z$ is not a unit. Then there is a unit in the first column of $f_{1}$. Then adding some left $\bar{\Gamma}$-multiple of some row-pair to the first row-pair will change $\left[\begin{array}{l}0 \\ z\end{array}\right]$ to $\left[\begin{array}{l}0 \\ 1\end{array}\right]$; and so by our "generator condition" some left $\bar{\Lambda}$-multiple of the lower row-pair can be used instead of the $\bar{\Gamma}$-multiple. Thus, in either case, we now have:

$$
f \sim\left[\begin{array}{ccc}
1 & 0 & \\
0 & 1 & * \\
* & & f_{2}
\end{array}\right] .
$$

Column-sweeping from left to right now yields:

$$
f \sim\left[\begin{array}{ccc}
1 & 0 & \\
0 & 1 & 0 \\
* & \hat{f}
\end{array}\right] .
$$

Since $f$ has an even number of rows and an odd number of columns, the same is true of $\hat{f}$. We can therefore iterate the above procedure until we get the upper triangular form

$$
f \sim\left[\begin{array}{cccccc}
1 & 0 & \cdots & 0 & 0 & 0 \\
0 & 1 & \cdots & 0 & 0 & 0 \\
\vdots & \vdots & \ddots & \vdots & \vdots & \vdots \\
* & * & \cdots & 1 & 0 & 0 \\
* & * & \cdots & 0 & 1 & 0 \\
* & * & \cdots & * & * & \hat{f}
\end{array}\right]
$$

where $\hat{f}$ is a column vector which contains a unit of $A$ and has an even number of rows. Once again we may use row-pair operations to put this column into the form $[1,0, \ldots, 0]^{\operatorname{tr}}(\operatorname{tr}=$ transpose $)$. For clarity in what follows, the following display 
shows the form of the resulting matrix in the particular situation $f \in A_{10 \times 7}$ (empty spaces denote zeros).

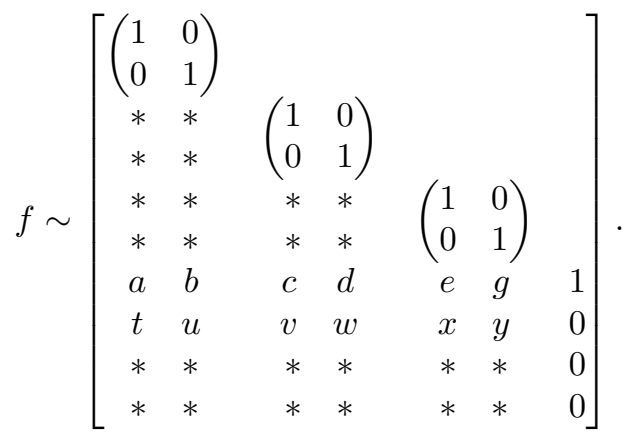

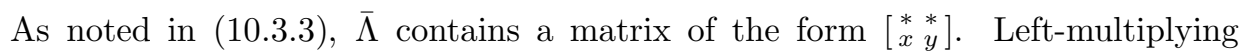
the third row-pair by $\left[\begin{array}{ll}* & * \\ x & y\end{array}\right]$ and subtracting the result from the row-pair below it changes $x$ and $y$ to zero. Similarly, we sweep downward with the second row-pair and change (the new) $v$ and $w$ to zero; and then sweep with the first row-pair to change $t$ and $u$ to zero. After this we sweep leftward with the last column, changing $a, b, c, d, e, g$ to zero.

A final interchange of row-pairs puts the row-pair we have just simplified at the bottom of the matrix. Our matrix now has the block-diagonal form

$$
f \sim\left[\begin{array}{cc}
h & 0 \\
0 & {\left[\begin{array}{l}
1 \\
0
\end{array}\right]}
\end{array}\right]
$$

and the proof is therefore complete.

Lemma 10.4. Let $D \subseteq E$ be an inclusion of artinian rings (with the same 1) such that $E$ is module-finite over $K=Z(D)$. Suppose further that $E$ is a simple ring with simple left module ${ }_{E} S$ and simple right module $T_{E}$. Then ${ }_{D} S$ is simple $\Leftrightarrow T_{D}$ is simple.

Proof. If either ${ }_{D} S$ or $T_{D}$ is simple, then the artinian $\operatorname{ring} D$, having a faithful simple module, is a simple ring. The simple left and right $D$-modules have the same (finite) dimension as $K$ vector spaces, and so do $S$ and $T$, the simple left and right $E$-modules. Consequently, if either ${ }_{D} S$ or $T_{D}$ is simple, then all four of the above $K$-dimensions are equal and both ${ }_{D} S$ and $T_{D}$ must be simple.

\section{LOCAL TFKS: EXPLICIT VERSION}

The purpose of this section is to convert the abstract description of the second exceptional class of $R$-orders $\Lambda$ satisfying TFKS, given in Local Necessity Theorem 9.6(ii)(b) [and in Theorem 1.3(c3)], to an explicit description. We use this to construct two examples, the second of which is not contained in Jacobinski's classification.

Notation 11.1. In this section we assume that $\Gamma$ is a PID that is module-finite over $R$. Hence $\Lambda$ is also an integral domain and $\Gamma$ is a maximal $R$-order in the division ring $\Lambda_{Q}=\Gamma_{Q}$. By Morita equivalence, this is no loss of generality since the most general exceptional rings $\Lambda$ in the previous paragraph are full matrix rings over integral domains. We also assume the following. 
(11.1.1) $\Gamma / J(\Gamma) \cong M_{2}(\Delta)$ for some division ring $\Delta$. In particular, the ring $\Gamma$ is matrix-local and $M=J(\Gamma)$ is its unique maximal ideal.

Note that (11.1.1) implies that $Z(\Gamma)$ is a local ring [LO, (4.1.6)], and then so is $Z(\Lambda)$ since $Z(\Gamma)$ is integral over $Z(\Lambda)$.

Our next theorem shows that the following properties of $\Lambda$ are equivalent in the presence of (11.1.1).

(11.1.2) (abstract description). $\Gamma(M \cap \Lambda) \Gamma=M$, and the simple left $\Gamma$-module remains simple as a $\Lambda$-module.

(11.1.3) (explicit description). $\Lambda$ contains an element of $M-M^{2}, \Delta$ has a divisionring extension $D$ of left (hence right) dimension 2 , and $\Lambda /(M \cap \Lambda) \cong D$ (as $R$-algebras).

Property (11.1.2) together with (11.1.1) is a restatement of Theorem 9.6(ii)(b).

Before proceding, we show why the left dimension of $D$ over $\Delta$ equals its right dimension in (11.1.3). Since $\Delta$ is a module-finite $R$-algebra, $\Delta$ is finite dimensional over $Z(\Delta)$, and therefore the left dimension of $D$ over $\Delta$ equals the quotient $Z(\Delta)-\operatorname{dim}(D) / Z(\Delta)-\operatorname{dim}(\Delta)$, a left-right symmetric expression.

Theorem 11.2. Let $\Gamma$ be a maximal $R$-order in a division ring ( $R$ semilocal) and suppose $\Gamma$ satisfies (11.1.1). Further suppose that $\Lambda$ is an $R$-order such that $\Lambda_{Q}=$ $\Gamma_{Q}$. Then (11.1.2) holds $\Longleftrightarrow(11.1 .3)$ holds.

Proof. $(\Leftarrow)$ Since we have (11.1.1), $\Gamma$ is matrix-local and its maximal ideal $J(\Gamma)$ has splitting number 2. Every (2-sided) ideal of any maximal order is a product of maximal ideals ([LO, 4.1.2], together with [R ' $75,(22.10)])$. Therefore every ideal of $\Gamma$ is a power of $J(\Gamma)$. In particular, this is true of $\Gamma(J(\Gamma) \cap \Lambda) \Gamma$. Since, by hypothesis, $\Lambda$ contains an element of $J(\Gamma)-J(\Gamma)^{2}$, we therefore have $\Gamma(J(\Gamma) \cap \Lambda) \Gamma=J(\Gamma)$, as desired.

Next we show that the simple left $\Gamma$-module $S$ remains simple as a $\Lambda$-module. We have $M_{2}(\Delta) \cong S^{2}$ as left $\Gamma$-modules. Since $M_{2}(\Delta)$ has dimension 2 as a left $D$ vector space, we see that $S$ has dimension 1 as a left $D$-vector space, a restatement of what we want to prove.

$(\Rightarrow)$ Let $D$ be the image of $\Lambda$ in $M_{2}(\Delta)$. Thus $D$ is an $R$-module of finite length [LO, 1.6(iii)] and therefore an artinian ring. The faithful simple left $M_{2}(\Delta)$-module $S$ is also a faithful $D$-module, and remains simple as a $D$-module by hypothesis. Since the artinian ring $D$ has a faithful simple module, $D$ is a simple ring. On the other hand, $\Lambda$ is a local ring, as shown in 9.7; and therefore its simple artinian homomorphic image $D$ is a division ring.

We now claim that there is an $R$-algebra monomorphism $\Delta \hookrightarrow D$. Consider the simple $\Gamma$-module $S=M_{2}(\Delta) \cdot\left[\begin{array}{l}1 \\ 0\end{array}\right]$. By hypotheses, this remains simple as a $\Lambda$ module, and therefore $D\left[\begin{array}{l}1 \\ 0\end{array}\right]=\Lambda\left[\begin{array}{l}1 \\ 0\end{array}\right]=S$. It follows that, for all elements $x, y \in \Delta$ there is an element $d \in D$ whose first column is $\left[\begin{array}{l}x \\ y\end{array}\right]$. In fact, there is a unique such element $d$ because the difference between any distinct two such elements would have first column zero, and would be an invertible matrix since $D$ is a division ring. For $\delta \in \Delta$ let $\phi(\delta)$ be the unique element of $D$ whose first column equals $\left[\begin{array}{l}\delta \\ 0\end{array}\right]$. This $\phi$ is clearly the desired $R$-algebra monomorphism.

The monomorphism $\phi: \Delta \hookrightarrow D$ is not a surjection since $D$ contains elements whose first column has the form $\left[\begin{array}{l}0 \\ y\end{array}\right]$ with $y \neq 0$. Therefore the dimension of $D$ over $\Delta$ equals 2 . 
To complete the proof it suffices to show that $\Lambda$ contains an element of $J(\Gamma)-$ $J(\Gamma)^{2}$. This follows from the hypothesis that $\Gamma(J(\Gamma) \cap \Lambda) \Gamma=J(\Gamma)$, together with the fact that all ideals of the matrix-local PID $\Gamma$ are powers of its unique maximal ideal $J(\Gamma)$.

The next lemma provides two maximal orders that can be used to construct rings conforming to explicit description (11.1.3). The first of these has a numbertheoretic origin, and the second has a ring-theoretic origin (but, in characteristic $p$, it becomes an order in a global field).

Lemma 11.3. (i) Let $\Upsilon$ be the maximal $\mathbb{Z}$-order in the division algebra of rational quaternions that contains $i, j, k$. Then, for any odd prime number $p$, the maximal $\mathbb{Z}_{p}$-algebra $\Gamma=\Upsilon_{p}$ satisfies condition (11.1.1), with $\Delta=\mathbb{Z} / p \mathbb{Z}$, and $\Delta$ has a field extension $D$ of dimension 2.

(ii) Let $\Upsilon=\Delta[x, \sigma]$, the skew polynomial ring over any field $\Delta$ such that $\Delta$ has an automorphism $\sigma$ of order 2 and a division ring extension $D$ of dimension 2, and let $G$ be the fixed field of $\sigma$. Then the center of $\Upsilon$ is the commutative polynomial ring $G\left[x^{2}\right]$, and for a suitable irreducible polynomial $f \in G\left[x^{2}\right]$ the maximal $G\left[x^{2}\right]_{f}$-order $\Gamma=\Upsilon_{f}$ satisfies condition (11.1.1), with $\Gamma / J(\Gamma)=$ $M_{2}(\Delta)$. (The subscript $f$ denotes the localization that inverts all irreducible polynomials in $G\left[x^{2}\right]$ except $f$.)

Proof. In situation (i) it is well-known that $\Upsilon / p \Upsilon \cong M_{2}(\Delta)$, with $\Delta=\mathbb{Z} / p \mathbb{Z}$. Thus $\Delta$ has a field extension $D$ of dimension 2 . The rest of statement (i) follows from the fact that the set of maximal ideals of any classical maximal order is in one-to-one correspondence with the set of maximal ideals of its center via contraction [LO, (4.1.6)].

In situation (ii) there exists a $G\left[x^{2}\right]$-algebra homomorphism of $\Upsilon$ onto $M_{2}(\Delta)$ [L '92, (3.2)]. The proof is completed, as above, by the correspondence of maximal ideals of maximal orders with those of their center.

The following is the simplest example of the type of $\operatorname{ring} \Lambda$ we are discussing.

Example 11.4. Choose any $\Gamma$ and $\Delta$ as in (11.1.1), where $\Delta$ has a division ring extension $D$ of left dimension 2 [for example, the rings mentioned in Lemma 11.3] and let $\nu$ be the natural surjection of $\Gamma$ onto $\Gamma / J(\Gamma)=M_{2}(\Delta)$. Choose any $R$ algebra embedding $D \subset M_{2}(\Delta)$. Then the pullback

$$
\Lambda=\{\lambda \in \Gamma \mid \nu(\lambda) \in D\}
$$

satisfies explicit condition (11.1.3), and therefore, by Theorem 11.2, satisfies the equivalent abstract condition (11.1.2). Note that, in this example, the largest conductor ideal for for $\Lambda$ and $\Gamma$ is $J(\Gamma)=J(\Lambda)$.

Note also that it is not always possible to choose $D$ to contain the set of diagonal matrices the form $\operatorname{diag}(\delta, \delta), \delta \in \Delta$. For example, take $\Gamma$ as in Lemma 11.3(ii), with $\Delta$ the complex numbers and $D$ the real quaternions. Then $D$ has no central subfield isomorphic to $\Delta$.

Example 11.5. Let $n$ be any positive integer. Then there is a ring $\Lambda$, as in Theorem 11.2, such that the largest conductor ideal for $\Lambda$ and $\Gamma$ is $J(\Gamma)^{n}$.

To construct such a $\Lambda$, let $\Gamma$ and $\Delta$ be as in Lemma 11.3(i) and choose a prime number $p$ such that -1 does not have a square root in $\Delta$, that is, $p \equiv 3(\bmod 4)$. Since $p$ is odd, we have $\Upsilon / p \Upsilon \cong M_{2}(\Delta)$ where $\Delta \cong \mathbb{Z} / p \mathbb{Z}$, and $J(\Gamma)=p \Gamma$ is the 
unique maximal ideal of $\Gamma$. We conclude from this that $\Gamma / J(\Gamma)^{n} \cong M_{n}\left(\mathbb{Z} / p^{n} \mathbb{Z}\right)$. Let $\nu$ be a ring homomorphism of $\Gamma$ onto $M_{n}\left(\mathbb{Z} / p^{n} \mathbb{Z}\right)$ with kernel $J(\Gamma)^{n}$, and set

$$
\Lambda=\left\{\lambda \in \Gamma \mid \nu(\lambda)=\left[\begin{array}{cc}
a & -b \\
b & a
\end{array}\right] \text { with } a, b \in \mathbb{Z} / p^{n} \mathbb{Z}\right\} .
$$

We prove that $\Lambda$ has the desired properties by checking the conditions in (11.1.1) and (11.1.3).

We already have (11.1.1) with $\Delta=\mathbb{Z} / p \mathbb{Z}$. Let $D$ be the set of matrices of the form $\left[\begin{array}{cc}x & y \\ -y & x\end{array}\right]$ in $M_{2}(\Delta)$. Since -1 has no square root in $\Delta, D$ is a field of dimension 2 over $\Delta$. Then $\Lambda /(J(\Gamma) \cap \Lambda) \cong D$ (as $\mathbb{Z}$-algebras). The only thing remaining to be checked is that $\Lambda$ contains an element $\gamma \in J(\Gamma)-J(\Gamma)^{2}$. We can take $\gamma$ to be any element of $\Gamma$ such that $\nu(\gamma)=\left[\begin{array}{cc}\pi & 0 \\ 0 & \pi\end{array}\right]$ where $\pi=p+p^{n} \mathbb{Z} \in \mathbb{Z} / p^{n} \mathbb{Z}$.

The orders $\Lambda$ in this last example are missing from Jacobinski's classification [J '74, Theorem 1(ii)] when $n>1$ because, in his terminology, $\Lambda$ is not a $\sigma$-order. See his definition of a sigma order on page 170. If $\Lambda$ were a $\sigma$-order, it would contain $J(\Gamma)$. To see that this does not hold, let $\gamma^{\prime}$ be an element of $\Gamma$ such that $\nu\left(\gamma^{\prime}\right)=\left[\begin{array}{ll}\pi & 0 \\ 0 & 0\end{array}\right]$, where $\pi=p+p^{n} \mathbb{Z}$. Then $\gamma^{\prime} \in J(\Gamma)$. But $\gamma^{\prime} \notin \Lambda$, as one sees from (11.5.1).

Acknowledgements. We wish to thank Roger Wiegand and the Referee for many, many helpful suggestions, criticisms, and corrections.

\section{REFERENCES}

[Ba '60] H. Bass, "Finitistic dimension and a homological generalization of semiprimary rings", Trans. Amer. Math. Soc. 95 (1960), 466-488. MR 28:1212

[Bo '72] N. Bourbaki, Commutative Algebra, Hermann, Paris, 1972. MR 50:12997

[CR '81] C. W. Curtis and I. Reiner, Methods of Representation Theory, with Applications to Finite Groups and Orders, vol. I, Wiley-Interscience, New York, 1981. MR 82i:20001

[E '73] E. G. Evans, Jr., "Krull-Schmidt and cancellation over local rings", Pac. J. Math., 46 (1973), 115-121. MR 48:2170

[GR '78] E. L. Green and I. Reiner, "Integral representations and diagrams", Mich. Math. J., 25 (1978), 53-84. MR 80g:16032

[Gr '65] A. Grothendieck, "Éléments de géométrie algébrique, IV", Publ. Math. IHES 24 (1965). MR 33:7330

[GL '88] R. M. Guralnick and L. S. Levy, "Presentations of modules when ideals need not be principal", Ill. J. Math 32 (1988), 593-653. MR 90c:16004

[GL '89] R. M. Guralnick and L. S. Levy, "Cancellation and direct summands in dimension 1", J. Algebra 142 (1991), 310-347. MR 92h:13012

[H '61] A. Heller, "On group representations over a valuation ring", Proc. Nat. Acad. of Sci. 47 (1961), 1194-1197. MR 23:A2468

[Ho '73] M. Hochster, "Non-openness of loci in noetherian rings", Duke Math J. 40 (1973), 215-219. MR 47:215

[J '68] H. Jacobinski, "Genera and decomposition of lattices over orders", Acta Math. 121 (1968), 1-29. MR 40:4294

[J '74] H. Jacobinski, "Unique decomposition of lattices over orders", in Representations of Algebras, Lecture notes \#488, Springer-Verlag, Berlin, 1974. MR 51:589; MR 52:3207

[Ja '43] N. Jacobson, The Theory of Rings, Amer. Math. Soc., 1943. MR 5:31f

[K '70] I. Kaplansky, Commutative Rings, Allyn and Bacon, Boston, 1970. MR 40:7234

[KL '90] L. Klingler and L. S. Levy, "Direct-sum cancellation: modules versus lattices", Comm. Alg. 18 (1990), 1857-1868. MR 91i:16014

[L '81] L. S. Levy, "Modules over pullbacks and subdirect sums", J. Algebra 70 (1981), 50-61. MR 82j:16052a

[L '92] L. S. Levy, "Direct-sum cancellation and genus class groups", in Methods in Module Theory, ed. G. Abrams, J. Haefner, K. Rangaswamy, Dekker, New York, 1992, 203-218. MR 94f: 16008 
[LO] L. S. Levy and C. J. Odenthal, "Package deal theorems and splitting orders, in dimension 1", Trans. Amer. Math. Soc. 348 (1996), 3457-3503.

[MR '87] J. C. McConnell and J. C. Robson, Noncommutative Noetherian Rings, WileyInterscience, Chichester, 1987. MR 89j:16023

[Re '61] I. Reiner, "The Krull-Schmidt theorem for integral group representations", Bull. Amer. Math. Soc. 67 (1961), 365-367. MR 25:2132

[Re '62] I. Reiner, "Failure of the Krull-Schmidt theorem for integral representations", Mich. Math J. 9 (1962), 225-231. MR 26:2482

[R 75] I. Reiner, Maximal Orders, Academic Press, New York, 1975. MR 52:13910

[Ro '69] K. Roggenkamp, "Das Krull-Schmidt Theorem für projektive Gitter über lokalen Ringen", Mitt. Math. Seminar Giessen 80 (1969), 29-50. MR 41:259

[W '80] R. B. Warfield, Jr., "Cancellation of modules and groups and stable range of endomorphism rings", Pac. J. Math., 91 (1980), 457-485. MR 82m:16022

[W '89] R. Wiegand, "Noetherian Rings of bounded representation type", in Proceedings of Microprogram on Commutative Algebra, MSRI, Berkeley, 1987, Springer, New York, 1989, 497-516. MR 90i:13010

Department of Mathematics, University of Wisconsin, Madison, Wisconsin 53706-1388

E-mail address: levy@math.wisc.edu

Department of Mathematics, Univerity of Toledo, Toledo, Ohio 43606-3390

E-mail address: codentha@math.utoledo.edu 